\section{UCDNN}

LIBRARY
University of Connecticut OpenCommons@UConn

8-12-2013

\title{
Electrothermal Properties of Perovskite Ferroelectric Films
}

Jialan Zhang

Univ. of Connecticut, flora8516@gmail.com

Follow this and additional works at: https://opencommons.uconn.edu/dissertations

\section{Recommended Citation}

Zhang, Jialan, "Electrothermal Properties of Perovskite Ferroelectric Films" (2013). Doctoral Dissertations. 158.

https://opencommons.uconn.edu/dissertations/158 


\title{
Electrothermal Properties of Perovskite Ferroelectric Films
}

\author{
Jialan Zhang, Ph.D. \\ University of Connecticut, 2013
}

The electrothermal (electrocaloric and pyroelectric) properties of ferroelectric thin films have many applications in active solid-state cooling and infrared sensing devices. It has been shown experimentally that some thin-film ferroelectrics can produce much larger electrothermal responses than their bulk counterparts. In this work, the electrothermal properties of bulk polar dielectric (ferroelectric and incipient ferroelectric) materials and thin films have been computed using a thermodynamic methodology and the effects of electrical, thermal and mechanical boundary conditions have been illustrated. In particular, the sensitivity of pyroelectric and electrocaloric response to bias and driving fields, lateral clamping and misfit strain, thermal stresses and composition have been demonstrated. The computations show that the electrothermal behavior of ferroelectric materials for practical cooling devices depends on a complex interplay of several related sets of physical phenomena. These include the nature of the ferroelectric transition, the particular dependence of the equilibrium and transport properties on electric field and mechanical boundary constraints, and the orientation and thermal expansion coefficients of the thin film and substrate materials. The combined results provide insights concerning how the composition and orientation of the thin film material, the choice of substrate, the deposition/annealing temperature, and the electrode configuration can be used to optimize the electrothermal properties for particular applications. 


\title{
Electrothermal Properties of Perovskite Ferroelectric Films
}

\author{
Jialan Zhang \\ B.S., Nanjing University, 2007
}

\author{
A Dissertation \\ Submitted in Partial Fulfillment of the \\ Requirements for the Degree of \\ Doctor of Philosophy \\ at the \\ University of Connecticut
}

2013 
Copyright by

Jialan Zhang

2013 


\section{APPROVAL PAGE}

\section{Doctor of Philosophy Dissertation}

\section{Electrothermal Properties of Perovskite Ferroelectric Films}

Presented by

Jialan Zhang, B.S.

Major Advisor

Dr. S. Pamir Alpay

Associate Advisor

Dr. George A. Rossetti, Jr.

Associate Advisor

Dr. Pu-Xian Gao

University of Connecticut

2013 


\section{ACKNOWLEDGEMENTS}

First of all, I would like to thank my advisor, Dr. S. Pamir Alpay. Dr. Alpay has been an outstanding advisor during my graduate studies at Uconn. I learned a lot from his patient guidance, excellent views and effective advice. I would also like to thank Dr. I. Burc Misirlioglu at Sabanci University for the great help and the discussion on domain structure during my stay in Turkey. I also want to thank my associate advisors Dr. George A. Rossetti, Jr. and Dr. Pu-Xian Gao for the useful discussion and guidance on ferroelectric materials and electrocaloric properties throughout my entire time at Uconn. I would like to thank my former and current research group and labmates: Dr. Shan Zhong, Dr. Gursel Akcay, Dr. Baris Okatan, Dr. Yong Liu, Dr. Claire Weiss Brennan, Dr. Liang Dong, Shawn Fonesca, Mehmet Tumerkan Kesim, Fu-Chang Sun, Hamidreza Khassaf, Dr. Adam Heitmann, Dr. Thevika Balakumar, Dr. Ching-Chang Chung, Richard Perez-Moyet, and Nasser Khakpash. I would like to thank the IMS staff and everyone that has collaborated with me on my research. Finally, I would like to thank my parents and extended family for their education, support and encouragement. I feel lucky to have all of you in my life. 


\section{TABLE OF CONTENTS}

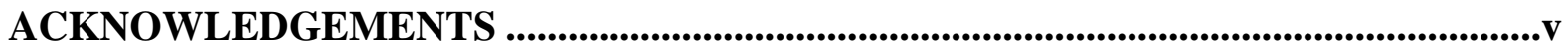

TABLE OF CONTENTS ........................................................................................... vi

LIST OF FIGURES ................................................................................................................. viii

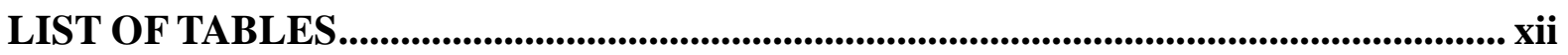

CHAPTER 1 INTRODUCTION ..............................................................................................1

1.1. Electrothermal (Electrocaloric \& Pyroelectric) Properties ..................................................1

1.2. Application of Electrothermal Properties of Ferroelectrics .................................................

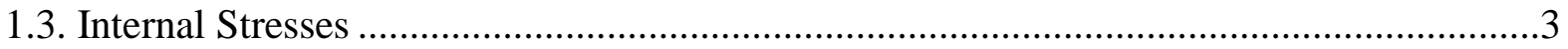

1.4. Perovskite-structured Ferroelectric Materials...................................................................

1.5. Early Research \& Recent Findings on Electrothermal Properties of Ferroelectrics............7

CHAPTER 2 THERMODYNAMIC BEHAVIOR OF ELECTROTHERMAL

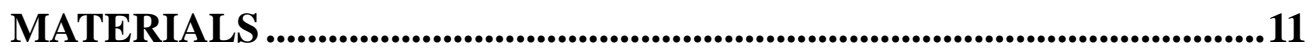

2.1. Electrothermal Coupling in Ferroelectric Materials .........................................................11

2.2. Electrocaloric Cooling Cycles .......................................................................................

2.3. Pyroelectric / Electrocaloric Property Coefficients .........................................................16

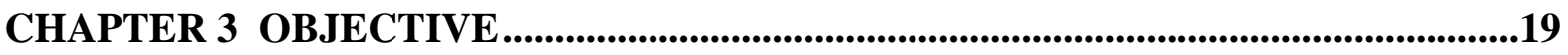

CHAPTER 4 THERMODYNAMIC MODEL ........................................................................20

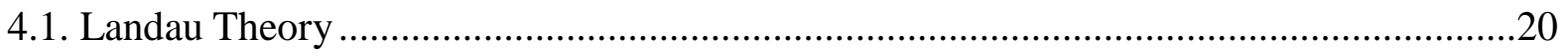

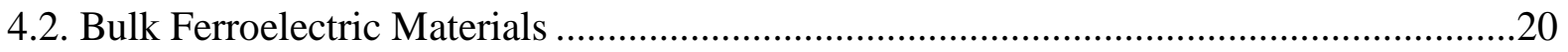

4.3. Epitaxial Films and Misfit Strain ...................................................................................22

4.4. Polycrystalline Films and Thermal Stresses ………..................................................26

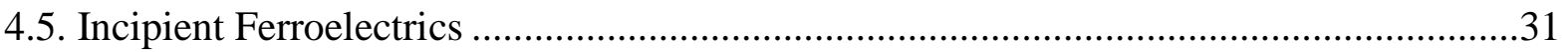


4.5.1. The Ferroelastic Transition

4.5.2. Strain-Induced Ferroelectric Phases

CHAPTER 5 INFLUENCE OF COMPOSITION, PHASE TRANSITION ORDER, AND MECHANICAL BOUNDARY CONDITION...................38

CHAPTER 6 INFLUENCE OF THERMAL STRESSES .........................................52

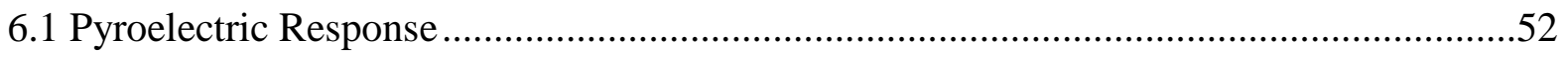

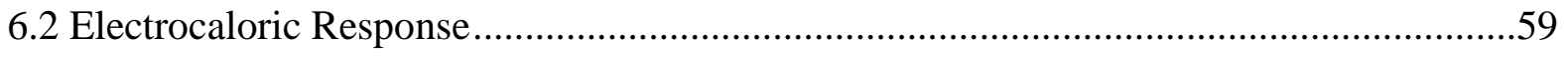

CHAPTER 7 INCIPIENT FERROELECTRIC MATERIALS ......................................65

CHAPTER 8 SUMMARY AND CONCULSIONS ......................................................74

8.1 Influence of Composition and Phase Transition Order .................................................74

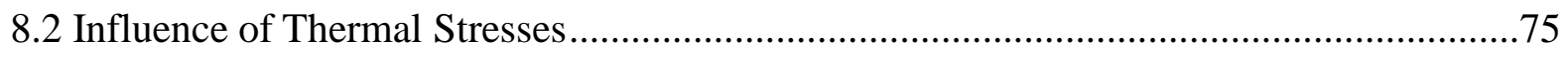

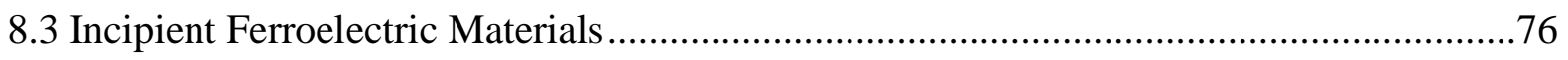

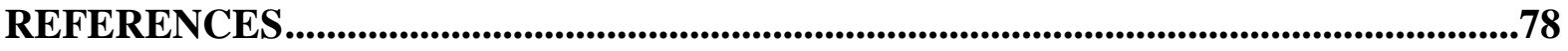




\section{LIST OF FIGURES}

Figure 1. Adiabatic temperature change $\Delta T$ (a) and corresponding change in energy density $C_{E} \Delta T$ (b) measured at different electric field levels for a PZT thin film, adapted from [39].

Figure 2. Schematic of an electrocaloric Ericsson cooling cycle.

Figure 3. Plots of polarization and specific heat as functions of temperature and applied electric field for monodomain $\mathrm{BaTiO}_{3}$ in the mechanically free

state [(a) and (b)], and under prefect lateral clamping [(c) and (d)].

Figure 4. Plots of polarization and specific heat as functions of temperature and applied electric field for monodomain $\mathrm{PbTiO}_{3}$ in the mechanically free state [(a) and (b)], and under prefect lateral clamping [(c) and (d)]

Figure 5. Plots of polarization and specific heat as functions of temperature and applied electric field for monodomain STO in the mechanically free state [(a) and (b)], and under prefect lateral clamping [(c) and (d)]...

Figure 6. Excess entropy as a function of temperature at different electric fields for (a) mechanically free (bulk) and (b) perfectly laterally clamped $\mathrm{BaTiO}_{3}$. At $E=0$ there is a discontinuity at the paraelectric-ferroelectric transition and as $E$ increases this discontinuity disappears. The vertical lines mark the temperatures where the maximum change in entropy occurs for an electric field change $\Delta E$ having differing values of the bias field $E_{a}$ and final field $E_{b}$ as shown by the arrows (see text for details).

Figure 7. Excess entropy as a function of temperature at different electric fields for (a) mechanically free (bulk) and (b) perfectly laterally clamped $\mathrm{PbTiO}_{3}$. 
Figure 8. Excess entropy as a function of temperature at different electric fields for (a) mechanically free (bulk) and (b) perfectly laterally clamped $\mathrm{SrTiO}_{3}$ near the structural phase transformation temperature.

Figure 9. Three-dimensional plots of the adiabatic temperature change $\Delta T$ as functions of $T$ and $\Delta E\left(E_{a}=50 \mathrm{kV} / \mathrm{cm}\right)$ for monodomain uniaxial (a), (c), and (e) unclamped stress-free (bulk) $\mathrm{BaTiO}_{3}, \mathrm{PbTiO}_{3}$, and $\mathrm{SrTiO}_{3}$, respectively, and (b), (d), and (f) $\mathrm{BaTiO}_{3}, \mathrm{PbTiO}_{3}$, and $\mathrm{SrTiO}_{3}$ thin films with $u_{m}=0$, respectively.

Figure 10. In-plane thermal strain of BST 70/30 films at RT as a function of the growth temperature on Si and sapphire along $a$ - and $c$ - axes.

Figure 11. The RT pyroelectric coefficient of BST films with composition (a) BST 60/40 (b) BST 70/30 (c) BST 80/20 (d) BST 90/10 as a function of the growth temperature and the applied electric field on Si substrates.

Figure 12. The RT pyroelectric coefficient of BST films with composition (a) BST 60/40 (b) BST 70/30 (c) BST 80/20 (d) BST 90/10 as a function of the growth temperature and the applied electric field on $c$-sapphire substrates .56

Figure 13. The RT pyroelectric coefficient of BST films with composition (a) BST 60/40 (b) BST 70/30 (c) BST 80/20 (d) BST 90/10 as a function of the growth temperature and the applied electric field on $a$-sapphire substrates. 
Figure 14. BST 70/30 on $c$-sapphire at RT: (a) The total polarization as a function of the growth temperature at various applied electric fields; (b) the total polarization for $T_{G}=100^{\circ} \mathrm{C}(\circ)$ and $T_{G}=700^{\circ} \mathrm{C}(\Delta)$ as a function of $E$; (c) the relative dielectric constant for $T_{G}=100^{\circ} \mathrm{C}(\circ)$ and $T_{G}=700^{\circ} \mathrm{C}(\Delta)$ as a function of $E$; and (d) the pyroelectric coefficient for $T_{G}=100^{\circ} \mathrm{C}(\mathrm{O})$ and $T_{G}=700^{\circ} \mathrm{C}(\Delta)$ as a function of $E$. .58

Figure 15. The variation in $u_{T}$ as a function of $T_{G}$ for a) BTO, b) BST 60/40, and c) PTO. 60

Figure 16. The dependence of $T_{C}$ on $T_{G}$ for BTO, BST 60/40, and PTO on Si and $c$ sapphire.

Figure 17. Adiabatic temperature change in (001) textured polycrystalline a) BTO, b) BST $60 / 40$ and c) PTO thin films $\left(E_{a}=50 \mathrm{kV} / \mathrm{cm}, \Delta E=120 \mathrm{kV} / \mathrm{cm}\right)$ as a function of $T_{G}$ on $\mathrm{Si}, c$-sapphire, and $a$-sapphire at RT.

Figure 18. Adiabatic temperature change in Bulk BTO and (001) textured polycrystalline BTO on $\mathrm{Si}\left(E_{a}=50 \mathrm{kV} / \mathrm{cm}, \Delta E=120 \mathrm{kV} / \mathrm{cm}\right)$ as a function of $T$ at different $T_{G}$

Figure 19. Misfit strain vs. temperature phase diagram of epitaxial monodomain (001) $\mathrm{SrTiO}_{3}$ films. The order parameters of the phases appearing in this map are: HT: $P_{1}=P_{2}=P_{3}=0, q_{1}=q_{2}=q_{3}=0$; ST: $P_{1}=P_{2}=P_{3}=0, q_{1}=q_{2}=0, q_{3} \neq 0$; FTI: $P_{1}=P_{2}=0, P_{3} \neq 0, q_{1}=q_{2}=q_{3}=0$; FTII: $P_{1}=P_{2}=0, P_{3} \neq 0, q_{1}=q_{2}=0, q_{3} \neq 0$; FOI: $\left|P_{1}\right|=\left|P_{2}\right| \neq 0, P_{3}=0, q_{1}=q_{2}=q_{3}=0$ 
Figure 20. (a) The adiabatic temperature change $\Delta T$ of an epitaxial (001) $\mathrm{SrTiO}_{3}$ film in a MIM configuration as a function of $u_{m}$ and $T$ for $E_{a}=50 \mathrm{kV} / \mathrm{cm}$ and $\Delta E=120 \mathrm{kV} / \mathrm{cm}$; (b) the out-of plane polarization $P_{3}$ as a function of temperature at $u_{m}=-0.02$ for $E_{a}=0 \mathrm{kV} / \mathrm{cm}$ and $E_{a}=50 \mathrm{kV} / \mathrm{cm}$.

Figure 21. The room-temperature adiabatic temperature change $\Delta T$ of epitaxial (001) $\mathrm{SrTiO}_{3}$ films with MIM ( $u_{m}=-0.020$, open squares) and [100]/[010] and [110] IDE configurations $\left(u_{m}=0.015\right.$, open circles and triangles, respectively) as a function of $\Delta E$ for $E_{a}=50 \mathrm{kV} / \mathrm{cm}$.

Figure 22. The adiabatic temperature change $\Delta T$ of an epitaxial (001) $\mathrm{SrTiO}_{3}$ film in a [100] IDE configuration as a function of $u_{m}$ and $T$ for $E_{a}=50 \mathrm{kV} / \mathrm{cm}$ and $\Delta E=120 \mathrm{kV} / \mathrm{cm}$.

Figure 23. The adiabatic temperature change $\Delta T$ of an epitaxial (001) $\mathrm{SrTiO}_{3}$ film in a [110] IDE configuration as a function of $u_{m}$ and $T$ for $E_{a}=50 \mathrm{kV} / \mathrm{cm}$ and $\Delta E=120 \mathrm{kV} / \mathrm{cm}$ .73 


\section{LIST OF TABLES}

Table 1. Primary pyroelectric / electrocaloric coefficients for several polar oxides

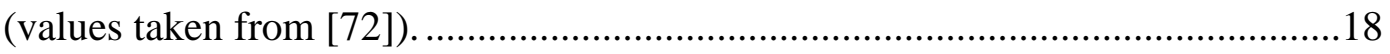

Table 2. Thermal expansion coefficients of Si and sapphire.............................................27

Table 3. Thermal expansion coefficients of BST as a function of Sr composition................27

Table 4. Bulk thermodynamic, elastic and electromechanical coefficients of BST...............30

Table 5. Landau Coefficients and Thermodynamic Properties of $\mathrm{BaTiO}_{3}$ and $\mathrm{PbTiO}_{3}$

(in SI units, the temperature $T$ in K) [23]. 


\section{CHAPTER 1}

\section{INTRODUCTION}

\subsection{Electrothermal (Electrocaloric \& Pyroelectric) Properties}

The electrothermal [electrocaloric and pyroelectric] properties stem from the coupling between the electrical and thermal properties of a polar dielectric solid. Electrocaloric effect is an adiabatic change in temperature $(\Delta T)$ in response to an applied electric field. Conversely, pyroelectric effect is the change in the charge density (polarization) in response to a chance in temperature $T$. Both electrocaloric and pyroelectric effect are described by the same property coefficient, $p=(\partial S / \partial E)_{T}=(\partial P / \partial T)_{E}$, where $S$ is entropy, $T$ is temperature, $E$ is electric field, and $P$ is the electric polarization. The property coefficient $p$ is non-zero only for crystals belonging to one of the ten polar point groups, in which there exists a unique polar axis. Ferroelectric crystals represent a sub-group of these wherein the spontaneous polarization may be reoriented among symmetry equivalent directions under the application of an electric field.

\subsection{Application of Electrothermal Properties of Ferroelectrics}

The electrothermal properties of ferroelectric materials have recently attracted considerable interest for use in a variety of applications such as solid-state cooling system and infra-red (IR) devices. Such devices may find applications, for example, in thin film micro-coolers used as thermal management systems for next generation integrated circuits (IC) or other high power density microelectronic components. Applications in IR devices include intruder alarms, fire detection, environmental monitoring, gas analysis, radiometers, laser detectors, and 2D arrays for uncooled thermal imaging. Ferroelectric materials are 
important for these applications due to their ability to be used successfully at ambient temperatures, thereby eliminating the need for expensive cooling systems [1], their relatively low cost, low power consumption, and wide operating range of temperature compared to photoconductive detectors [2]. Furthermore, they are eco-friendly compared with conventional domestic and industrial refrigeration which produces greenhouse gases heavily. The temperature change resulting from the electrocaloric effect is analogous to adiabatic demagnetization, which has long been used to cool cryogenic systems to temperatures approaching absolute zero [3]. Like magnetocaloric materials, solid-state coolers based on electrocaloric materials may provide efficiency, size, weight, and device integration benefits relative to conventional vapor compression systems. Electrocaloric materials based on ferroelectrics are known to have high electrothermal conversion factors $(\sim 0.95)$ and a maximum in response that can be varied over a wide temperature range ( $100 \mathrm{~K}$ to $800 \mathrm{~K})$ according to the temperature of the ferroelectric Curie point [4]. The ease of applying electric as opposed to magnetic fields makes electrocaloric coolers especially attractive for cooling device applications.

There are essentially two modes of operation for pyroelectric IR sensors. One mode operates at a temperature below (but typically near) the Curie temperature $T_{C}$ of the ferroelectric where the variation in the spontaneous polarization as a function of temperature is large. In the dielectric bolometer mode, one works slightly above $T_{C}$ in the paraelectric state of the ferroelectric and with an applied bias field to induce polarization [5-9]. In either case, it is required that the ferroelectric is deposited in a thin film form on an IC compatible substrate. 


\subsection{Internal Stresses}

Internal stresses arise from the lattice mismatch between the film and the substrate, the difference in coefficients of thermal expansion (CTEs) of the film and the substrate, and the self-strain of the paraelectric-ferroelectric phase transformation.

For on-chip cooling applications or pyroelectric IR sensors, thin film ferroelectrics must be deposited on an IC compatible substrate. The growth of ferroelectric films on ICfriendly substrates such as $\mathrm{Si}$ or sapphire $\left(\alpha-\mathrm{Al}_{2} \mathrm{O}_{3}\right)$ typically employ industry-standard deposition techniques such as sputtering or metal-organic solution deposition, and the resultant ferroelectric film is usually polycrystalline. For such films, in-plane strains arise from thermal stresses due to the thermal expansion mismatch between the film and the substrate, and also from the self-strain of the paraelectric-ferroelectric phase transformation if the film growth is carried out at temperatures above $T_{C}$.

\subsection{Perovskite-structured Ferroelectric Materials}

The structural families comprising perovskites, Aurivillius phases, and tungsten bronzes admit a very wide range of isomorphous ion substitutions, making it possible to adjust the crystal lattice parameters, the Curie temperature, and the order and nature of the paraelectric to ferroelectric transition. Relaxor ferroelectric materials, in particular, are expected to exhibit unusually high electrocaloric properties due to the configurational entropy change associated with the large change in macroscopic polarization induced by modest electric field strengths. For relaxor ferroelectric materials, the electrocaloric effect arising due to the spontaneous loss of macroscopic polarization on lifting of the field becomes analogous to adiabatic demagnetization in magnetocaloric materials based on super- 
paramagnetic phenomena [3]. All of these attributes make perovskite and layer-structured ferroelectric materials especially appealing as objects for investigations of electrothermal coupling in thin film ferroelectrics. The potential for finding lead-free materials makes Aurivillius phase and tungsten bronze materials particularly attractive. Alternatively, a series of reports on polyvinylidene fluoride (PVDF)-based ferroelectric copolymer and terpolymer films have shown electrocaloric effects that are comparable to those observed in ceramic materials [10]. The nature of the ferroelectric transition in these materials may also be varied from normal to relaxor behavior.

$\mathrm{BaTiO}_{3}(\mathrm{BTO})$ is a prototypical perovskite ferroelectric material with $T_{C}=120^{\circ} \mathrm{C}$. The paraelectric-ferroelectric transition temperature can be reduced by adding $\mathrm{Sr}^{2+}$ which substitutes for the $\mathrm{Ba}^{2+}$ cations in the $\mathrm{ABO}_{3}$ lattice. As such, barium strontium titanate $\left(\mathrm{Ba}_{x} \mathrm{Si}_{1-x} \mathrm{TiO}_{3}, \mathrm{BST}\right)$ is an attractive material system that has received a great deal of interest in several device applications, in particular for dielectrically tunable phase shifters in telecommunications [11].

It has also been demonstrated experimentally that high-quality BST films with good dielectric properties can be grown on Pt-Si and other IC-friendly substrates. For example, Cole et al. measured a real (relative) permittivity of 348 and a dissipation factor of 0.024 in BST 60/40 thin films doped with $1 \mathrm{~mol} \% \mathrm{Mg}$ at $10 \mathrm{GHz}$ [12]. Pervez et al. demonstrated that high tunability (over 13:1) BST films capable of sustaining short-duration fields greater than $4 \mathrm{MV} / \mathrm{cm}$ deposited on $c$-plane sapphire $\left(\alpha-\mathrm{Al}_{2} \mathrm{O}_{3}\right)$ can be obtained by varying the $\mathrm{O}_{2}$-partial pressure [13]. The effect of strain on dielectric properties of either epitaxial or nonepitaxial BST films on different substrates (including Pt-Si and $\mathrm{Al}_{2} \mathrm{O}_{3}$ ) were studied as well [14-17]. Hu et al. found that Sn-doped BST thin films on $\mathrm{LaNiO}_{3}$-coated $\mathrm{Si}$ substrate displayed a 
relatively low dielectric constant (less than 200 at frequency higher than $100 \mathrm{~Hz}$ ) but had low dielectric loss (less than $1 \%$ at $100 \mathrm{~Hz}$ ) and improved leakage current characteristics [18]. In addition, thin films of $\mathrm{Ba}\left(\mathrm{Ti}_{\mathrm{x}} \mathrm{Sn}_{1-\mathrm{x}}\right) \mathrm{O}_{3}, \mathrm{BST} / \mathrm{SiN}$ bilayers, and BST films with extremely small grain size ( $25 \mathrm{~nm}$ ) on IC-friendly substrates were also investigated recently [19-21].

$\mathrm{SrTiO}_{3}$ (STO) is an incipient ferroelectric material which undergoes a ferroelastic phase transition from the prototypical cubic perovskite $\left[\mathrm{Pm} \overline{3} \mathrm{~m}\left(\mathrm{O}_{\mathrm{h}}{ }^{1}\right)\right]$ to a tetragonal [I4/mcm $\left.\left(\mathrm{D}_{4 \mathrm{~h}}{ }^{18}\right)\right]$ structure at $-168^{\circ} \mathrm{C}$ that is brought about by the rotations of $\mathrm{TiO}_{6}$ octahedra about the cubic axes. Extensive studies in the 1960s and 1970s attribute this phase transition to a $\Gamma_{25}$ soft-phonon mode instability at the [111] zone boundary [22-24]. Although STO crystals or polycrystalline ceramics remains paraelectric down to absolute zero, the ferroelectric phase can be induced by pressure [25], electrical field [26], doping [27], and through equi-biaxial in-plane misfit strains in epitaxial STO films [28,29]. A thermodynamic analysis by Pertsev et al. $[29,30]$ has shown that it is possible to induce a variety of different ferroelectric phases in epitaxial thin films of STO that are not stable in monolithic singlecrystal or polycrystalline forms. Following this work, ferroelectricity at room temperature ( $\mathrm{RT} \cong 300 \mathrm{~K}$ ) in epitaxial (001) STO thin films was observed experimentally by carefully adjusting the equi-biaxial in-plane misfit strain [28]. One of the important features of the Pertsev phase diagram is that it shows that the misfit strain can be used to access two or three different ferroelectric phases depending on the temperature (between $\sim 150 \mathrm{~K}<\mathrm{T}<\sim 350 \mathrm{~K}$ ). By adjusting the sign and magnitude of the misfit strain, it was predicted that ferroelectric states with out-of-plane or in-plane spontaneous polarizations (along [001] and [100]/[010] of the STO film, respectively) can be generated $[29,30]$. Because the derivative of the ferroelectric polarization with respect to temperature typically shows a sharp maximum near 
a paraelectric to ferroelectric phase transitions, an enhancement in the electrocaloric response can be expected.

There is a significant interest in STO thin films as a replacement for the traditional gate oxide material $\mathrm{SiO}_{2}$ [31], in dynamic random access memories (DRAMs) [32], and tunable microwave telecommunication devices due to their high dielectric response ( 300 in STO single-crystals) and its strongly non-linear applied electric field dependence [33,34]. It is, therefore, crucial to understand the role of thermal stresses on the dielectric properties of STO thin films, especially for films synthesized using industry-standard deposition techniques (such as sputtering or metal-organic solution deposition) which usually produce polycrystalline STO films. Based on previous experimental work and theoretical studies discussed above, it is clear that BST films are ideally suited for applications in IR detection. It was found that the pyro-response of BST 60/40 epitaxial films on $\mathrm{Si}$ substrate was suppressed compared to bulk BST 60/40 due to internal stresses [35]. By taking into account internal stresses as well as the formation of misfit dislocations at the growth temperature, the effects of the film thickness and operating temperature on the pyroelectric coefficients of epitaxial (001) $\mathrm{Ba}_{0.6} \mathrm{Si}_{0.4} \mathrm{TiO}_{3}$ (BST 60/40) and $\mathrm{Pb}_{0.5} \mathrm{Zr}_{0.5} \mathrm{TiO}_{3}$ (PZT 50/50) thin films on different substrates were investigated [36]. It was shown that the pyroelectric coefficients increased with increasing film thickness for these films on (001) $\mathrm{LaAlO}_{3}$ and 0.29. $\mathrm{LaAlO}_{3}: 0.35 \cdot \mathrm{Sr}_{2} \mathrm{TaAlO}_{6}$ (LSAT) substrates due to stress relaxation by misfit dislocations. These studies show that an optimum pyroelectric response can be realized by tuning the operating temperature and the film thickness to adjust the internal stresses. Using a similar approach, Cao et al. investigated pyroelectric and dielectric properties of epitaxial BST 70/30 thin films on anisotropic substrates [37]. Considering the direct relation between 
the pyroelectric and electrocaloric coefficients, we have recently adopted our theoretical formalism [38] to describe the giant electrocaloric properties of ferroelectric films as well [39].

Previous results from our group show that internal stresses in ferroelectric thin films could significantly deteriorate the functional properties of ferroelectric films and an optimum pyroelectric and electrocaloric response can be realized by tuning various experimental parameters to adjust the internal stresses [40,41]. For polycrystalline ferroelectric films produced by industry-standard deposition techniques such as sputtering or metal-organic solution deposition [42], internal stresses mainly come from the CTE mismatch between the film and the substrate and the self-strain of the paraelectric-ferroelectric transition. Therefore, thermal stresses may have a profound influence on the electrothermal responses of ferroelectric thin films.

\subsection{Early Research \& Recent Findings on Electrothermal Properties of Ferroelectrics}

Although ferroelectric materials have attracted attention for use in solid-state coolers and pyroelectric energy harvesters since the 1960's [43-45], these early measurements on polycrystalline ferroelectric ceramics at low electric fields produced only small adiabatic temperature changes $(\Delta T<2 \mathrm{~K})$ at temperatures close to the Curie temperature $T_{C}$. Interest in electrocaloric cooling systems was revitalized in 2006 when it was demonstrated that thin film perovskite-structured ferroelectrics with low defect densities could support field strengths of $1000 \mathrm{kVcm}^{-1}$, producing a two order of magnitude increase in adiabatic temperature change, $\Delta T>10 \mathrm{~K}$ [39]. Experimental data demonstrating the observed 'giant'

electrocaloric $\Delta T$ and the corresponding reversible change in energy density, $Q^{\mathrm{rev}}=C_{E} \Delta T$ 
(where $C_{E}$ is the specific heat at constant electric field), are shown for a lead zirconatetitanate $\left[\mathrm{Pb}(\mathrm{Zr}, \mathrm{Ti}) \mathrm{O}_{3}, \mathrm{PZT}\right]$ thin film in Figure 1.
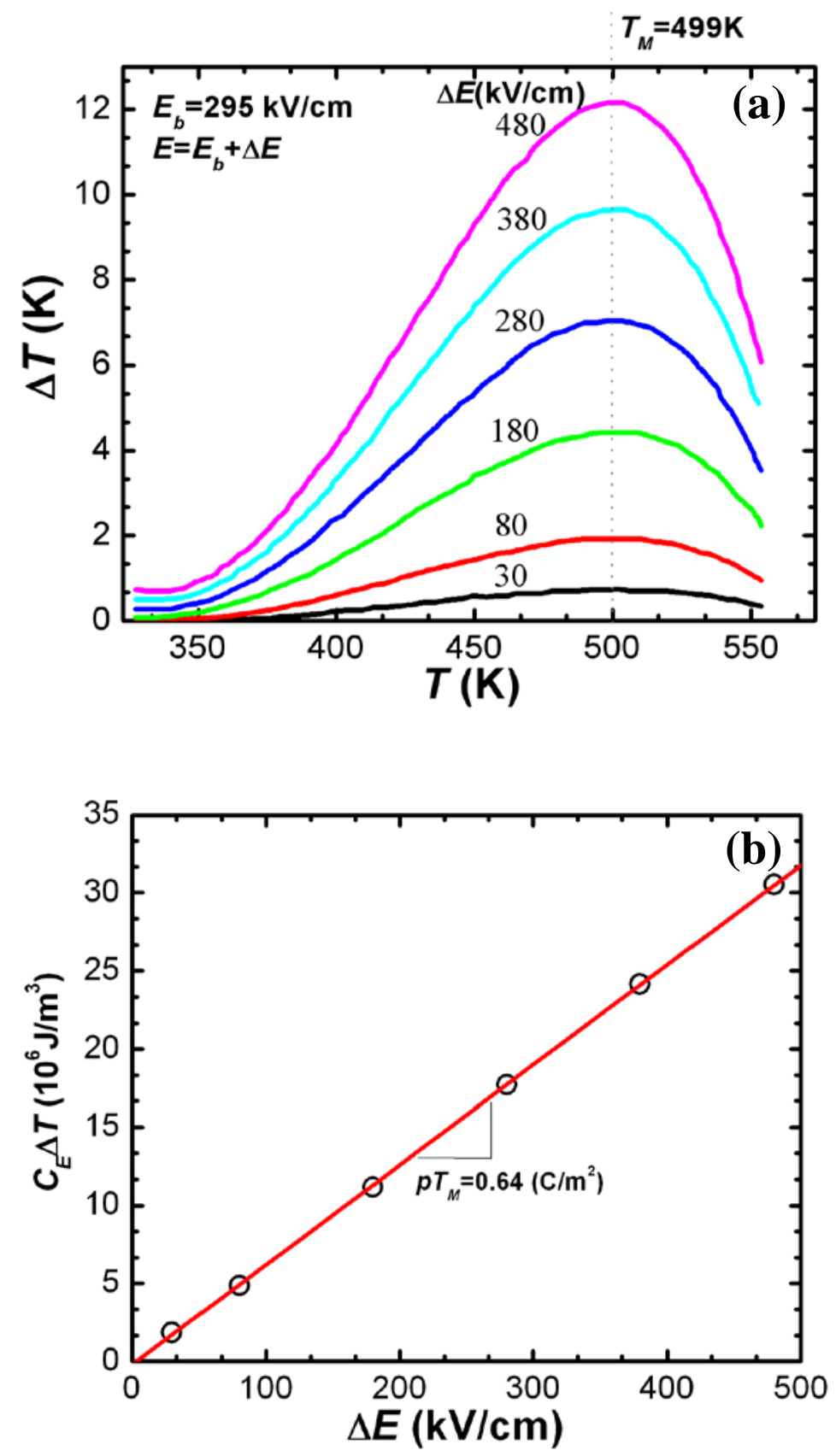

Figure 1. Adiabatic temperature change $\Delta T$ (a) and corresponding change in energy density $C_{E} \Delta T$ (b) measured at different electric field levels for a PZT thin film, adapted from [39]. 
These findings immediately stimulated a resurgence of interest in the electrocaloric properties of ferroelectric materials. Since this time, the literature on both experimental and theoretical aspects of the topic has been steadily growing [46-49]. Thermodynamic models combining the Maxwell relations and the Landau theory of phase transformations [21,46,50,51], molecular dynamics [52], phase-field approaches [53], Monte Carlo simulations [54], and first-principles calculations [55] have all been used to understand the origins of the electrocaloric effect in different types of ferroelectric materials and to model their electrocaloric properties under different choices of electrical, thermal and mechanical boundary conditions. Moreover, large electrocaloric effects have very recently been reported in some ferroelectric copolymer systems, such as poly (vinylidene fluoride-trifluoroethylene) [P(VDF-TrFE)] [10], inviting new possibilities for the development of composite or other hybrid materials systems.

Thermodynamic analyses and constitutive modeling carried out on thin film ferroelectric materials (which will be discussed in detail in Chapter 4) showed that that the large $\Delta T$ as observed experimentally at high electric field strengths is, in fact, intrinsic to the strain-free film [46,50,56]. The observed and computed pyroelectric coefficients are comparable, $p \cong-1200 \mathrm{Cm}^{-2} \mathrm{~K}^{-1}$ near the maximum temperature $\left(T_{M}\right)$, a value that is typical of ferroelectric materials near their Curie points. Since that time, numerous theoretical and experimental studies confirming these findings have been carried out [10,48,57-61] wherein it has been found that the electrocaloric temperature changes reported for ferroelectric thin films are comparable to those reported for thermoelectric-based thin film coolers [62]. As a consequence, optimization of the chemistry, structure, deposition and architecture of 
ferroelectric thin films may now offer the potential to make electrocaloric materials competitive with thermoelectric materials for active solid-state cooling applications.

Indeed, advances in the growth and processing of epitaxial thin films, polycrystalline ferroelectric ceramics and single crystals, and ferroelectric polymers that achieve low defect densities now make it possible to attain much higher field strengths in these materials. At the same time, progress in the application of dielectric materials to high energy density capacitors, thin film pyroelectric sensing and memory devices, and single crystal piezoelectric transducers provide numerous possibilities for achieving trade-offs among materials properties, form factors, and fabrication processes suitable for electrocaloric devices. High capacity thin-film architectures involving inter-digitated or other electrode configurations enabling the application of high electric fields while simultaneously reducing volumetric requirements can be envisioned. If realized, solid-state cooling devices could offer significant advantages when compared to thermoelectric-based technologies.

Because practical electrocaloric materials exhibit a broad range of ferroelectric behaviors, to be successfully employed in practical cooling devices, these complex phenomena in ferroelectric materials require investigation using both computational and experimental approaches. A thermodynamic methodology that can be used to identify the influence of factors controlling the electrocaloric and pyroelectric performance of thin film materials, including Curie temperature, phase change characteristics, constitutive properties and external parameters of electrical and mechanical boundary conditions is described below. 


\section{CHAPTER 2}

\section{THERMODYNAMIC BEHAVIOR OF ELECTROTHERMAL MATERIALS}

\subsection{Electrothermal Coupling in Ferroelectric Materials}

The coupling between electric and thermal fields in polar dielectric solids gives rise to the electrothermal effects. In ferroelectric materials, the direction of the polarization vector $\boldsymbol{P}$ can be reoriented among symmetry equivalent crystallographic directions under the application of an electric field, $\boldsymbol{E}$. Large electrocaloric effects are expected in ferroelectric materials because of the strong temperature variation of the modulus $P=|\boldsymbol{P}|$ that occurs near the Curie temperature, or alternatively, due to changes in both the modulus and direction of $\boldsymbol{P}$ near the temperatures of any inter-ferroelectric phase transitions that may occur between different symmetry ferroelectric phases. In general, the application of an electric field $\boldsymbol{E}$ conjugate to the polarization vector $\boldsymbol{P}$ destroys these phase changes. However, for suitably small field strengths a temperature interval exists for which the contribution of the phase change to the temperature derivative $(d P / d T)_{E}$ does not vanish, giving rise to large electrocaloric effects.

The reversible change of internal energy $(d U)$ in an elastic dielectric solid follows from the first and second laws of thermodynamics,

$$
d U=T d S+\sigma_{i j} d u_{i j}+E_{i} d D_{i}
$$

where $T, \sigma_{i j}$ are temperature and external stress, and $d S$, $d u_{i j}$, and $d D_{i}$ are the changes in entropy, strain, and dielectric displacement, respectively. The dielectric displacement $D_{i}$ is given by the constitutive relation, 


$$
D_{i}=\varepsilon_{0} E_{i}+P_{i}
$$

where $\varepsilon_{0}$ is the dielectric permittivity of vacuum. The total scalar free energy density describing the polar dielectric solid is

$$
F=U-T S-\sigma_{i j} u_{i j}-E_{i} D_{i}
$$

and taking the differential of $F$ and substituting Eq. (1) into (3) yields,

$$
d F=-S d T-u_{i j} d \sigma_{i j}-D_{i} d E_{i}
$$

As $F$ is an exact differential, we have,

$$
d F=\left(\frac{\partial F}{\partial T}\right)_{\sigma, E} d T+\left(\frac{\partial F}{\partial \sigma_{i j}}\right)_{T, E} d \sigma_{i j}+\left(\frac{\partial F}{\partial E_{i}}\right)_{T, \sigma} d E_{i} .
$$

Eqs. (4) and (5) give relations for the thermal, elastic, and dielectric properties of the system,

$$
\left(\frac{\partial F}{\partial T}\right)_{\sigma, E}=-S,\left(\frac{\partial F}{\partial \sigma_{i j}}\right)_{T, E}=-u_{i j},\left(\frac{\partial F}{\partial E_{i}}\right)_{T, \sigma}=-D_{i}
$$

Differentiating the set of Eqs. (6), Maxwell relations relating the material compliances of the system are obtained, e.g.:

$$
\begin{aligned}
& -\left(\frac{\partial^{2} F}{\partial T \partial u_{i j}}\right)_{E}=\left(\frac{\partial S}{\partial u_{i j}}\right)_{E, T}=\left(\frac{\partial \sigma_{i j}}{\partial T}\right)_{\sigma, E} \\
& -\left(\frac{\partial^{2} F}{\partial \sigma_{i j} \partial E_{i}}\right)_{T}=\left(\frac{\partial u_{i j}}{\partial E_{i}}\right)_{\sigma, T}=\left(\frac{\partial D_{i}}{\partial \sigma_{i j}}\right)_{E, T} \\
& -\left(\frac{\partial^{2} F}{\partial E_{i} \partial T}\right)_{\sigma}=\left(\frac{\partial D_{i}}{\partial T}\right)_{\sigma, E}=\left(\frac{\partial S}{\partial E_{i}}\right)_{\sigma, T}
\end{aligned}
$$


The last relation gives the definition of the pyroelectric coefficient, and is valid for all polar dielectric solids.

$$
\left(p_{i}\right)_{\sigma}=\left(\frac{\partial^{2} F}{\partial E_{i} \partial T}\right)_{\sigma}=\left(\frac{\partial D_{i}}{\partial T}\right)_{\sigma, E}=\left(\frac{\partial S}{\partial E_{i}}\right)_{\sigma, T}
$$

For a ferroelectric material, the adiabatic temperature change $\Delta T$ corresponding to a field change of magnitude $\Delta E=E_{b}-E_{a}$ can then be explicitly calculated by integration of Eq. (8):

$$
\Delta T\left(T, E_{i}, \sigma\right)=-\int_{E_{a}}^{E_{b}} \frac{T}{C_{E}\left(T, E_{i}, \sigma\right)}\left(\frac{\partial P_{i}\left(T, E_{i}, \sigma\right)}{\partial T}\right)_{E} d E_{i}
$$

The partial derivative on the right hand side of Eq. (9) is primarily controlled by the nature of the ferroelectric transition and plays an important role in determining the electrothermal coupling properties of ferroelectric materials. In thin film structures, the order of the ferroelectric transition may be changed by the chemical composition, as in solid solution ferroelectrics, and/or by a change in the mechanical boundary conditions arising from heteroepitaxial and thermal expansion mismatch with the substrate [41,63-66]. In general, it is expected that adjusting conditions to produce a transition lying close to a tricritical point (i.e., a crossover from first-order to second-order behavior) will always produce the largest equilibrium electrocaloric response, since the derivative $(d P / d T) \rightarrow \infty$ at this point [67]. 


\subsection{Electrocaloric Cooling Cycles}

Electrocaloric materials employed in practical cooling device applications must make use of an appropriate thermodynamic cycle. One example is the Ericsson cycle depicted schematically in Figure 2. The cycle consists of two constant field steps at $E_{a}$ and $E_{b}$ and two isothermal steps at $T_{1}$ and $T_{2}$. If the electrocaloric material is operated under conditions corresponding to the maximum in response at $T_{M}$ as shown in Figure 1, the reversible energy density change occurring as a result of a field change $\Delta E=E_{b}-E_{a}$ reduces to $Q^{r e v} \cong T_{M} p \Delta E$. The right-hand panel of Figure 1 shows that this simple relation is approximately obeyed during a field step corresponding to one-half the cycle depicted in Figure 2, for example, the step corresponding to the segment $B \rightarrow C$. A polar dielectric of volume $V$ operates as a heat pump when a time varying electric field $E_{i}(t)$ having extrema with values $E_{b}$ and $E_{a}$ is impressed on its surfaces. Because the electrocaloric temperature change that results is a volume effect over $V$, the cycle along the path $A \rightarrow B \rightarrow C \rightarrow D$ can produce useful cooling at the heat source in a bulk material only if the heat flux is rectified. Several methods of doing so utilizing micro-electro-mechanical (MEMS) or thermoelectric thermal switches located at the heat source and sink have been proposed [68-71], with each switch being alternately open or closed during one-half cycle of operation. 


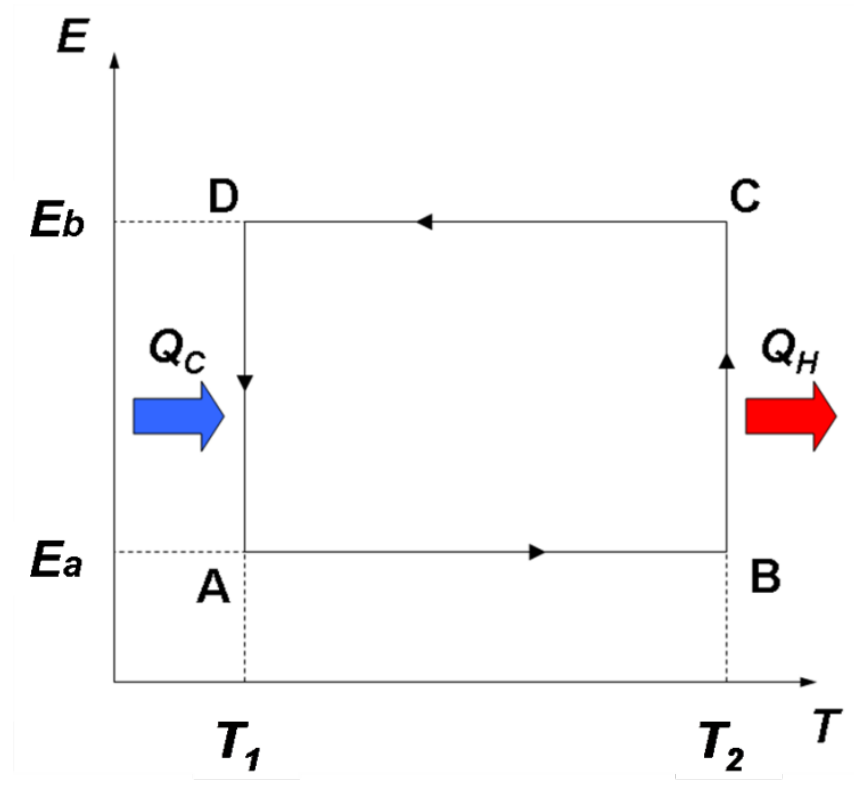

Figure 2. Schematic of an electrocaloric Ericsson cooling cycle.

The particular form of the function $E_{i}(t)$ is expected to depend on the details of the cooling device design. For example, $E_{i}(t)$ may be a sine, a saw tooth, or a square wave. The impression of $E_{i}(t)$ on the surfaces of the dielectric will produce a time varying temperature field $T\left(r_{i}, t\right)$ that varies spatially with position, $r_{i}$, and exhibits a phase lag with respect to $E_{i}(t)$. Considering a propagation direction perpendicular to the plane of a thin plate of polar dielectric material having isothermal surfaces, and assuming for simplicity that $T\left(r_{i}, t\right)$ is a sinusoidal thermal wave of wavelength $\lambda$, the frequency $v$ of the thermal wave is given by the solution of the thermal conduction equation. For these boundary conditions this is,

$$
v\left(T, E_{i}\right) \approx \frac{k\left(T, E_{i}\right)}{\bar{C}\left(T, E_{i}\right) l^{2}}=\frac{D\left(T, E_{i}\right)}{l^{2}}
$$

where $k\left(T, E_{i}\right)$ is the thermal conductivity, $\bar{C}\left(T, E_{i}\right)$ is the volume specific heat, $D\left(T, E_{i}\right)$ is the thermal diffusivity, and $l$ is the thickness of the polar dielectric material. The cooling 
capacity, П, achieved during the cycle $A \rightarrow B \rightarrow C \rightarrow D \rightarrow A$ per unit volume of dielectric $V$ becomes,

$$
\Pi\left(T, E_{i}\right) \approx \phi W v\left(T, E_{i}\right)=\frac{\phi W D\left(T, E_{i}\right)}{l^{2}}
$$

where $W$ is the work of the cycle and $\phi$ is a measure of the material losses, including specifically conduction losses and the dissipative dielectric losses, $\tan \delta=\varepsilon$ "' $\varepsilon$ ' where $\varepsilon$ ' and $\varepsilon "$ are the real and imaginary parts of the dielectric permittivity, respectively.

Although the above analysis is highly simplified, it illustrates the influence of the specific heat and thermal conductivity of the polar dielectric material in determining the cooling capacity of practical electrocaloric cooling devices. An understanding of the dependences of these properties on temperature and applied electric field is expected to be an area of future research in the development of electrocaloric materials for cooling devices.

\subsection{Pyroelectric / Electrocaloric Property Coefficients}

In polar dielectric solids, the center of positive charge does not coincide with the center of negative charge. The electrothermal properties of polar dielectric crystals are determined by the internal atomic rearrangements in the structure that lead to a change in the separation of the charge centers subject to a uniform change in temperature. Such rearrangements may occur under conditions of constant strain $\left(u_{i j}\right)$ such that there is no change in the unit cell dimensions, and this change gives rise to the primary pyroelectric effect characterized by the coefficient $\left(p_{i}\right)_{u}$. If the crystal is not mechanically clamped, the unit cell dimensions may change due to thermal expansion. Further, because all pyroelectric materials lack a center of symmetry, they are also piezoelectric. The secondary pyroelectric 
coefficient $\left(p_{i}\right)_{\sigma}$ that is usually measured by experiment on bulk materials at constant stress $\left(\sigma_{i j}\right)$ includes both of these contributions. The two coefficients are not independent, and the primary coefficient is given by the thermodynamic relation,

$$
\left(p_{i}\right)_{u}=\left(p_{i}\right)_{\sigma}-a_{j k}^{E} c_{j k l m}^{E, \sigma} d_{i l m}^{\sigma}
$$

where $a_{j k}^{E}$ is the coefficient of thermal expansion, $c_{j k l m}^{E, \sigma}$ is the elastic stiffness, and $d_{i l m}^{\sigma}$ is the piezoelectric compliance.

It is apparent from Eq. (12) that large primary electrocaloric/pyroelectric effects are favored in crystal structures that have weak interatomic binding forces characterized by large amplitude thermal motion and small elastic stiffness. For example, perovskite oxides have corner-sharing oxygen octahedral linkages with small central cations and so tend to exhibit large primary coefficients when compared to more close-packed polar crystal structures such as wurtzite. Table 1 compares the room-temperature values of the primary electrocaloric/pyroelectric coefficients for several different polar oxides and the archetypal polymer ferroelectric, polyvinylidene fluoride. In ferroelectric materials the especially strong temperature dependence of the coefficient $\left(p_{i}\right)_{\sigma}$ arises due to the proximity of a displacive structural instability, where the polarization near the phase change varies with temperature and is proportional to the amplitude of a soft transverse optic phonon mode that condenses at the Curie temperature, $T_{C}$. A general constitutive model for materials having these differing phase change characteristics is outlined below. 
Table 1. Primary pyroelectric / electrocaloric coefficients for several polar oxides (values taken from [72]).

\begin{tabular}{|c|c|c|c|}
\hline $\begin{array}{c}\text { Polar } \\
\text { Dielectric }\end{array}$ & $\begin{array}{l}\text { Crystal } \\
\text { Structure }\end{array}$ & $\begin{array}{c}\text { Crystal } \\
\text { Symmetry }\end{array}$ & $\begin{array}{c}\left(p_{i}\right)_{u} @ 298 K \\
\left(\mu C \mathrm{~m}^{-2} K^{-1}\right)\end{array}$ \\
\hline \multicolumn{4}{|l|}{ Poled Ceramics } \\
\hline $\mathrm{BaTiO}_{3}$ & Perovskite & Tetragonal, $4 \mathrm{~mm} / \infty \mathrm{m}$ & $\square-260$ \\
\hline $\mathrm{Pb}\left(\mathrm{Zr}_{0.95} \mathrm{Ti}_{0.05}\right) \mathrm{O}_{3}$ & Perovskite & Trigonal, $3 \mathrm{~m} / \infty \mathrm{m}$ & $\square-306$ \\
\hline \multicolumn{4}{|l|}{ Crystals } \\
\hline $\mathrm{LiNbO}_{3}$ & $\begin{array}{l}\text { Corundum } \\
\text { derivative }\end{array}$ & Trigonal, $3 m$ & $\square-96$ \\
\hline $\mathrm{LiTaO}_{3}$ & $\begin{array}{l}\text { Corundum } \\
\text { derivative }\end{array}$ & Trigonal, $3 m$ & $\square-175$ \\
\hline$\left(\mathrm{Ba}_{0.5} \mathrm{Sr}_{0.5}\right) \mathrm{Nb}_{2} \mathrm{O}_{6}$ & Tungsten Bronze & Tetragonal, $4 \mathrm{~mm}$ & $\square-502$ \\
\hline $\mathrm{SrBi}_{2} \mathrm{Ta}_{2} \mathrm{O}_{9}$ & Aurivillius & Orthorhombic, $m m 2$ & -240 \\
\hline $\mathrm{ZnO}$ & Wurtzite & Hexagonal, 6mm & $\square-6.9$ \\
\hline PVDF & Molecular Solid & Orthorhombic, $m m 2$ & $\square-40$ \\
\hline
\end{tabular}




\section{CHAPTER 3}

\section{OBJECTIVE}

The Ph.D. research that was carried out establishes a comprehensive understanding of the electrothermal properties and provides a quantitative analysis by taking into account the effects of:

- $\quad$ Phase transition orders and compositions.

- Different electrical and mechanical boundary conditions.

- $\quad$ Internal stresses (thermal \& epitaxial).

This work strives to answer the following questions:

- What are the electrothermal properties of mechanically free (bulk) ferroelectric perovskites and 'incipient' ferroelectrics? This establishes a reference state.

- What are the electrothermal properties of ferroelectric perovskites and 'incipient' ferroelectric under the simplest boundary condition: perfect laterally clamping with no misfit strain? The results will be compared with the reference state to quantify the influence of the mechanical boundary conditions.

- How do thermal stresses influence the electrothermal properties of ferroelectrics? We will analyze polycrystalline ferroelectric films on various substrates as examples to determine the influence of thermal stresses.

- What are the electrocaloric and pyroelectric properties of epitaxial incipient ferroelectrics?

The results will serve as a guide to experimental studies to maximize electrocaloric and pyroelectric properties of ferroelectric thin films. 


\section{CHAPTER 4}

\section{THERMODYNAMIC MODEL}

\subsection{Landau Theory}

The Gibbs free energy density of a proper ferroic phase transformation of a singledomain system with a three-component order parameter, $\eta$, can be written in general form as:

$$
\begin{aligned}
F(\eta, T, x)= & \int_{V}\left[\alpha_{i j} \eta_{i} \eta_{j}+\beta_{i j k l} \eta_{i} \eta_{j} \eta_{k} \eta_{l}+\ldots \ldots .+A_{i j k l}\left(\nabla_{i} \eta_{j} \cdot \nabla_{k} \eta_{l}\right)+\delta_{i j k} x_{i j} \eta_{k}\right. \\
& \left.+\frac{1}{2} q_{i j k l} x_{i j} \eta_{k} \eta_{l}+\frac{1}{2} C_{i j k l} x_{i j} x_{k l}-\frac{1}{2} \Omega_{i}(\text { int }) \eta_{i}-\Omega_{i} \eta_{i}\right] d V
\end{aligned}
$$

where $\alpha_{i j}$, $\beta_{i j k l}$ are the free energy expansion coefficients, $A_{i j k l}$ coefficient of the gradient term, $\delta_{i j k}$ and $q_{i j k l}$ are the bilinear and linear-quadratic coupling coefficients between the order parameter and the strain, $x_{i j}$, $C_{i j k l}$ are the elastic coefficients, $\Omega_{i}$ is an external field conjugate to the order parameter, and $\Omega_{i}$ (int) is the internal field due to variations in $\eta_{i}$. Proper ferroelectric phase transformations can be described by the above relation with the ferroelectric polarization $\boldsymbol{P}$ as the order parameter and $\boldsymbol{E}$ as the conjugate field.

\subsection{Bulk Ferroelectric Materials}

The starting point for the analysis of the electrothermal properties of perovskite ferroelectrics such as $\mathrm{BaTiO}_{3}$ and $\mathrm{PbTiO}_{3}$ (PTO) is the classical 2-4-6 Ginzburg-LandauDevonshire polynomial [73,74] expressed in powers of the polarization vector $\boldsymbol{P}=\left\{P_{1}, P_{2}\right.$,

$\left.P_{3}\right\}$. Assuming isothermal conditions and considering only the paraelectric-ferroelectric 
transition between the cubic (Pm3m) and tetragonal (P4mm) phases, the expansion of the free energy density for the monodomain single crystal in the unconstrained state reduces to

$$
F_{\text {bulk }}(T, E, P)=F_{0}+\alpha_{1} P^{2}+\alpha_{11} P^{4}+\alpha_{111} P^{6}-E P_{i}
$$

where $F_{0}$ is the free energy density of the paraelectric phase. In the tetragonal ferroelectric state $P_{1}=P_{2}=0$, and $P_{3}=P \neq 0$ is the component of the electric polarization directed along one of the cube axes of the high-symmetry phase. Here $E=E_{3}$ is a component of the applied electric field vector oriented parallel to the polarization direction, and $\alpha_{1}, \alpha_{11}$, and $\alpha_{111}$ are dielectric stiffness coefficients. The quadratic coefficient $\alpha_{1}$ is given by the Curie-Weiss Law, $\alpha_{1}=\alpha_{0}\left(T-T_{C}\right)$, where $\alpha_{0}=1 /\left(2 \varepsilon_{0} C\right), \varepsilon_{0}$ is the permittivity of free space, $T_{C}$ is the Curie temperature, and $C$ is the Curie-Weiss constant. The higher-order dielectric stiffness coefficients $\alpha_{11}$ and $\alpha_{111}$ are, in principle, also analytical functions of temperature. However, to reproduce the qualitative features of the paraelectric-ferroelectric phase change at temperatures close to the Curie point, where the Landau series is asymptotically accurate, it is sufficient to take these higher-order coefficients as constants.

When $E=0$, the spontaneous polarization $\left(P_{S}\right)$ in the tetragonal ferroelectric phases of $\mathrm{BaTiO}_{3}$ and $\mathrm{PbTiO}_{3}$ follows from the condition of thermodynamic equilibrium,

$$
\frac{\partial F_{\text {bulk }}}{\partial P}=\alpha_{1}+2 \alpha_{11} P^{2}+3 \alpha_{111} P^{4}=0
$$

or 


$$
P_{S}^{2}(T)=\frac{-\alpha_{11}+\sqrt{\alpha_{11}^{2}-3 \alpha_{1} \alpha_{111}}}{3 \alpha_{111}}
$$

When $E \neq 0$, the equilibrium polarization $P^{0}$ has contributions that arise from both the spontaneous polarization and the induced polarization, and its value is determined from the equation of state,

$$
\partial F_{b u l k} / \partial P=E
$$

\subsection{Epitaxial Films and Misfit Strain}

For epitaxial thin film materials, the free energy density has to be modified to take into account the clamping effect of the substrate and/or the misfit strain, um, due to the lattice mismatch between the film and substrate. Here we consider as an example a particular case

of epitaxial $\mathrm{BaTiO}_{3}, \mathrm{PbTiO}_{3}$ films deposited on a cubic substrate with (001) film // (001) substrate. Considering the mechanical boundary conditions for this situation, [i.e., equal inplane biaxial stress components (in contracted notation) $\sigma_{1}=\sigma_{2}$, no shear stresses $\left(\sigma_{4}=\sigma_{5}=\sigma_{6}=0\right)$ and no out-of-plane stress $\left.\left(\sigma_{3}=0\right)\right]$, the free energy density for the ferroelectrics $\mathrm{BaTiO}_{3}$ and $\mathrm{PbTiO}_{3}$ can be expressed as,

$$
F_{\text {film }}\left(T, E, P, u_{m}\right)=F_{0}+\alpha_{1} P^{2}+\alpha_{11} P^{4}+\alpha_{111} P^{6}-E P+F_{e l}
$$


The elastic energy term, $F_{e l}$, is given by,

$$
F_{e l}=\tilde{C}\left(u_{m}-Q_{12} P^{2}\right)^{2}
$$

Here, $u_{m}$ is the in-plane polarization-free misfit strain defined as:

$$
u_{m}=\frac{a_{\text {substrate }}-a_{\text {film }}}{a_{\text {substrate }}}
$$

where $a_{\text {film }}$ and $a_{\text {substrate }}$ are the pseudocubic lattice parameters of the free standing film and the substrate, respectively. The product $Q_{12} P^{2}$ is the self-strain due to polarization, $Q_{i j}$ are the cubic electrostrictive coefficients in polarization notation, and $\tilde{C}$ is an effective elastic modulus,

$$
\tilde{C}=C_{11}+C_{12}-\frac{2 C_{12}^{2}}{C_{11}}
$$

where $C_{i j}$ are the elastic stiffnesses at constant polarization. After some rearrangement, the following expression obtains [65]:

$$
F_{\text {film }}\left(T, E, P, u_{m}\right)=F_{0}+\tilde{\alpha}_{1} P^{2}+\tilde{\alpha}_{11} P^{4}+\alpha_{111} P^{6}-E P+u_{m}^{2} \tilde{C}
$$

with modified dielectric stiffness coefficients given by: 


$$
\begin{gathered}
\tilde{\alpha}_{1}=\alpha_{1}-2 u_{m} Q_{12} \tilde{C} \\
\tilde{\alpha}_{11}=\alpha_{11}+Q_{12}^{2} \tilde{C}
\end{gathered}
$$

It should be noted if we consider an epitaxial film with no in-plane strain $\left(u_{m}=0\right)$, the Curie temperatures $T_{C}$ of the ferroelectrics $\mathrm{BaTiO}_{3}$ and $\mathrm{PbTiO}_{3}$ will not change, relative to their values in the unclamped state, since $\tilde{\alpha}_{1}=\alpha_{1}$ in Eq. (23). However, the order of the phase transformation may be changed due to the two-dimensional clamping of the film by the substrate as described by $\tilde{\alpha}_{11}$, which is not a function of the misfit strain $u_{m}$. Hence, if the phase transformation in the unconstrained single crystal is of first-order (i.e., $\alpha_{11}<0$ ), the phase transformation in the corresponding epitaxial film may be of second-order, depending on the magnitude of $Q_{12}^{2} \widetilde{C}$. When the external field $E=0$, minimization of the modified free energy with respect to the polarization $\left(\partial F_{\text {film }} / \partial P=0\right)$ yields the spontaneous polarization of the film as a function of the misfit strain,

$$
P_{S}^{2}\left(T, u_{m}\right)=\frac{-\tilde{\alpha}_{11}+\sqrt{\tilde{\alpha}_{11}^{2}-3 \tilde{\alpha}_{1} \alpha_{111}}}{3 \alpha_{111}}
$$

We limit our discussion here only to the phase transformation from a cubic non-polar to a tetragonal ferroelectric “c-domain” phase. As shown theoretically for $\mathrm{BaTiO}_{3}$ and $\mathrm{PbTiO}_{3}$, other tetragonal variants and non-tetragonal ferroelectric phases may also form, depending on the sign and magnitude of the misfit strain [75]. These can be included by modifying the Landau potential to take into account all components of the polarization vector and the corresponding elastic/electrostrictive energies associated with the additional 
polarization components. Furthermore, the theoretical approach may also be expanded to include polydomain formation as well [76].

It follows from Eq. (13) that for a constant electric field $E$, the excess entropy $S^{X S}$ and the excess specific heat $\Delta C_{E}$ of the thin film material can be expressed through:

$$
\begin{gathered}
S_{E, \sigma}^{X S}\left(T, E, u_{m}\right)=-\left(\frac{\partial F^{0}}{\partial T}\right)_{E, \sigma} \\
\Delta C_{E, \sigma}\left(T, E, u_{m}\right)=-T\left(\frac{\partial^{2} F^{0}}{\partial T^{2}}\right)_{E, \sigma}
\end{gathered}
$$

where $F^{0}$ is the equilibrium total free energy density. The adiabatic temperature change can then be determined explicitly from the relation,

$$
\Delta T\left(T, E, u_{m}\right)=-\int_{E_{a}}^{E_{b}} \frac{T}{C_{E, \sigma}\left(T, E, u_{m}\right)}\left(\frac{\partial P\left(T, E, u_{m}\right)}{\partial T}\right)_{E} d E
$$

where the total volumetric specific heat $C_{E, \sigma}\left(T, E, u_{m}\right)$ is estimated by adding the computed excess specific heat to the zero-field values of the "hard mode" contributions taken from experimental measurements. The pyroelectric coefficient can then be expressed as:

$$
p=\frac{d P^{0}(E=0)}{d T}+\int_{0}^{E}\left(\frac{\partial \varepsilon}{\partial T}\right)_{E} d E
$$

where $\varepsilon=\left(\partial^{2} F / \partial P^{2}\right)^{-1}$ is the dielectric response. 


\subsection{Polycrystalline Films and Thermal Stresses}

For polycrystalline ferroelectric films, thermal stresses can develop in the film due to the differences in the coefficients of thermal expansion (CTEs) between the film and the substrate upon cooling from the film growth (or annealing) temperature, $T_{G}$. A variety of substrates $\left(\mathrm{SrTiO}_{3}, \mathrm{MgO}, \mathrm{LaAlO}_{3}\right.$, metallized $\mathrm{Si}$ and sapphire) can be used to provide different levels of internal stresses. For some device applications, such as active on-chip cooling, integrated circuit (IC)-friendly substrates are required. In these situations, the values of the misfit strain, $u_{m}$, in the preceding equations need to be replaced by in-plane thermal strain, $u_{T}$, given by,

$$
u_{T}\left(T, T_{G}\right)=\int_{T}^{T_{G}} \alpha_{F} d T-\int_{T}^{T_{G}} \alpha_{S} d T
$$

where $\alpha_{F}$ and $\alpha_{S}$ are the in-plane coefficients of thermal expansion of the film and the substrate, respectively, $T$ is the temperature of the film, and $T_{G}$ is the growth/annealing temperature. The way $T_{G}$ is defined in this analysis depends on the film deposition technique. For physical vapor deposition methods such as pulsed laser deposition (PLD), $T_{G}$ is substrate temperature during growth. For chemical deposition techniques such as spin coating or metal-organic chemical vapor deposition (MOCVD), $T_{G}$ corresponds to the temperature at which the final annealing step is carried out. The CTEs of the film materials (BST 60/40, BST 70/30, BST 80/20, and BST 90/10) and the substrates [(001) Si, $c$-sapphire, and $a$ sapphire] used in our calculations are given in Table 2 and Table 3. 
Table 2. Thermal expansion coefficients of $\mathrm{Si}$ and sapphire.

\begin{tabular}{ccc}
\hline \hline Substrate & TEC of the substrate, $\times 10^{6}\left({ }^{\circ} C^{-1}, T\right.$ in $\left.{ }^{\circ} C\right)$ & Ref. \\
\hline Si & $3.725 \times\left\{1-\exp \left[-5.88 \times 10^{-3}(T+149)\right]\right\}+5.548 \times 10^{-4} \times(T+273)$ & {$[77]$} \\
sapphire $\boldsymbol{c}$-axis & $8.026+8.17 \times 10^{-4} \times T-3.279 \times \exp \left(-2.91 \times 10^{-3} \times T\right)$ & {$[78]$} \\
sapphire $\boldsymbol{a}$-axis & $7.419+6.43 \times 10^{-4} \times T-3.211 \times \exp \left(-2.59 \times 10^{-3} \times T\right)$ & \\
\hline
\end{tabular}

Table 3. Thermal expansion coefficients of BST as a function of Sr composition.

\begin{tabular}{lc}
\hline \hline & TEC, $\times 10^{6}\left({ }^{\circ} C^{-1}, T\right.$ in $\left.{ }^{\circ} C\right)$ \\
\hline BST 60/40 & $8.90+7.90 \times 10^{-3} \times T$ \\
BST 70/30 & $8.81+8.58 \times 10^{-3} \times T$ \\
BST 80/20 & $8.72+9.26 \times 10^{-3} \times T$ \\
BST 90/10 & $8.63+9.94 \times 10^{-3} \times T$ \\
\hline \hline
\end{tabular}

For perovskite ferroelectrics such as $\mathrm{BaTiO}_{3}$ and $\mathrm{PbTiO}_{3}$ deposited on (001) substrates such as Si and c-sapphire, which have isotropic in-plane coefficients of thermal expansion, the thermal strains are equibiaxial. For perovskite ferroelectrics such as $\mathrm{BaTiO}_{3}$ and $\mathrm{PbTiO}_{3}$ deposited on substrates such as $a$-sapphire, however, the in-plane thermal strains are anisotropic, and the in-plane strains along $a$ - and $c$-axes are $u_{T 1}\left(T_{G}\right)$ and $u_{T 2}\left(T_{G}\right)$, respectively.

Assuming that the thickness of the substrate is much larger than the film thickness so that all internal stresses are concentrated in the film, for BST films on Si and $c$-sapphire 
substrates which have isotropic in-plane CTEs, the free energy density of the film taking into account the in-plane thermal stresses can be expressed as [79]:

$$
G_{\text {iso }}\left(P, T, u_{T}, E\right)=\tilde{\alpha}_{1} P^{2}+\tilde{\alpha}_{11} P^{4}+\alpha_{111} P^{6}+\frac{u_{T}^{2}}{S_{11}+S_{12}}-E P
$$

The modified dielectric stiffness coefficients $\tilde{\alpha}_{1}$ and $\tilde{\alpha}_{11}$ are given by:

$$
\begin{aligned}
& \tilde{\alpha}_{1}=\alpha_{1}-u_{T} \frac{2 Q_{12}}{S_{11}+S_{12}} \\
& \tilde{\alpha}_{11}=\alpha_{11}+\frac{Q_{12}^{2}}{S_{11}+S_{12}}
\end{aligned}
$$

where $S_{11}$ and $S_{12}$ are the elastic compliances of the film at constant polarization. It is assumed that the thermodynamic, elastic and electromechanical coefficients of the BST films are a linear function of composition determined by averaging corresponding values of $\mathrm{BaTiO}_{3}$ and $\mathrm{SrTiO}_{3}$ (Table 4).

The condition for thermodynamic equilibrium is given by the equation of state $\partial G_{\text {iso }} / \partial P=E$ such that:

$$
\frac{\partial G_{i s o}}{\partial P}=2 \tilde{\alpha}_{1} P+4 \tilde{\alpha}_{11} P^{3}+6 \alpha_{111} P^{5}=E
$$

Thus, the dielectric response along [001] is: 


$$
\varepsilon_{\text {iso }}=\left(\frac{\partial^{2} G_{i s o}}{\partial P^{2}}\right)^{-1}=\frac{1}{2\left(\tilde{\alpha}_{1}+6 \tilde{\alpha}_{11} P^{2}+15 \alpha_{111} P^{4}\right)}
$$

and the pyroelectric coefficient along [001] can be expressed as: $[1,80]$

$$
p_{\text {iso }}=\frac{d P_{S}}{d T}+\int_{0}^{E}\left(\frac{\partial \varepsilon_{\text {iso }}}{\partial T}\right)_{E} d E
$$

where $P_{S}$ is the spontaneous polarization along [001] corresponding to the solution of $\partial G_{\text {iso }} / \partial P=0$, and $T$ is the operating temperature of the pyroelectric. The first term in Eq. (7) is purely from the spontaneous polarization in the ferroelectric phase below $T_{C}$ and the second term provides the pyroelectric contribution from the temperature variation of the induced polarization.

For $a$-sapphire substrate which has anisotropic in-plane CTEs along its $a$ - and $c$ - axes, the in-plane strains due to the thermal stresses along $a$ - and $c$ - axes are different. The free energy density taking into account the in-plane thermal stresses can be expressed as:

$$
G_{\text {aniso }}\left(P, T, u_{T 1}, u_{T 2}, E\right)=\tilde{\alpha}_{3} P^{2}+\tilde{\alpha}_{33} P^{4}+\alpha_{111} P^{6}+\frac{S_{11}\left(u_{T 1}^{2}+u_{T 2}^{2}\right)}{2\left(S_{11}^{2}-S_{12}^{2}\right)}-E P
$$

where $u_{T 1}\left(T_{G}\right)$ and $u_{T 2}\left(T_{G}\right)$ are the in-plane strains along $a$ - and $c$-axes, respectively, and the modified dielectric stiffness coefficients $\tilde{\alpha}_{3}$ and $\tilde{\alpha}_{33}$ are given by [81]: 


$$
\begin{gathered}
\tilde{\alpha}_{3}=\alpha_{1}-\frac{Q_{12}}{S_{11}+S_{12}}\left(u_{T 1}+u_{T 2}\right), \text { and } \\
\tilde{\alpha}_{33}=\alpha_{11}+\frac{Q_{12}^{2}}{S_{11}+S_{12}}
\end{gathered}
$$

The equation of state is $\partial G_{a n i s o} / \partial P=E$ from which the polarization and its dependence on $u_{T 1}, u_{T 2}$, and $E$ can be determined. The dielectric and pyroelectric coefficients follow from Eqs. (35) and (36) with appropriate changes in the re-normalized Landau coefficients [Eqs. (38) and (39)].

Table 4. Bulk thermodynamic, elastic and electromechanical coefficients of BST.

\begin{tabular}{ccccc}
\hline & BST 60/40 & BST 70/30 & BST 80/20 & BST 90/10 \\
\hline$T_{C}\left({ }^{\circ} \mathrm{C}\right)$ & -29.2 & 8.1 & 45.4 & 82.7 \\
$C\left({ }^{\circ} \mathrm{C}\right)$ & $1.34 \times 10^{5}$ & $1.43 \times 10^{5}$ & $1.52 \times 10^{5}$ & $1.61 \times 10^{5}$ \\
$Q_{11}\left(\mathrm{~m}^{4} / \mathrm{C}^{2}\right)$ & 0.0843 & 0.0907 & 0.0971 & 0.104 \\
$Q_{12}\left(\mathrm{~m}^{4} / \mathrm{C}^{2}\right)$ & -0.0324 & -0.0356 & -0.0387 & -0.0419 \\
$C_{11}\left(\mathrm{~N} / \mathrm{m}^{2}\right)$ & $2.33 \times 10^{11}$ & $2.19 \times 10^{11}$ & $2.04 \times 10^{11}$ & $1.90 \times 10^{11}$ \\
$C_{12}\left(\mathrm{~N} / \mathrm{m}^{2}\right)$ & $9.18 \times 10^{10}$ & $9.00 \times 10^{10}$ & $8.82 \times 10^{10}$ & $8.64 \times 10^{10}$ \\
$\alpha_{11}\left(\mathrm{~N} \mathrm{~m} / \mathrm{C}^{4}\right)$ & $2.16 \times 10^{6} \times T$ & $2.52 \times 10^{6} \times T$ & $2.88 \times 10^{6} \times T$ & $3.24 \times 10^{6} \times T$ \\
& $+3.02 \times 10^{8}$ & $+6.9 \times 10^{7}$ & $-1.64 \times 10^{8}$ & $-3.97 \times 10^{8}$ \\
$\alpha_{111}\left(\mathrm{~N} \mathrm{~m}{ }^{10} / \mathrm{C}^{6}\right)$ & $3.96 \times 10^{9}$ & $4.62 \times 10^{9}$ & $5.28 \times 10^{9}$ & $5.94 \times 10^{9}$ \\
\hline \hline
\end{tabular}




\subsection{Incipient Ferroelectrics}

The thermodynamic analysis described above can also be extended to incipient ferroelectrics, such as strontium titanate, $\mathrm{SrTiO}_{3}$. Although $\mathrm{SrTiO}_{3}$ crystals or polycrystalline ceramics remain paraelectric down to $0 \mathrm{~K}$, the ferroelectric phase can be induced by uniaxial stress [25], an external electrical field [26], or by doping [27]. A thermodynamic analysis by Pertsev et al. $[29,30]$ has shown that it is possible to induce a variety of different ferroelectric phases in epitaxial thin films of $\mathrm{SrTiO}_{3}$ that are not stable in monolithic single-crystal or polycrystalline forms. Following this work, ferroelectricity at room temperature (RT $\cong 300 \mathrm{~K})$ in epitaxial (001) $\mathrm{SrTiO}_{3}$ thin films was observed experimentally by carefully adjusting the equi-biaxial in-plane misfit strain [28]. Here we describe a thermodynamic analysis for the electrocaloric properties of $\mathrm{SrTiO}_{3}$ films subject to mechanical boundary conditions that involve both perfect clamping and misfit strain. This analysis is summarized in the sections below.

\subsubsection{The Ferroelastic Transition}

Under stress-free conditions, a structural phase transformation in $\mathrm{SrTiO}_{3}$ takes place below the temperature $T_{s t}$ to a centrosymmetric tetragonal phase belonging to space group I4/mcm. This ferroelastic transition involves a rotation of the oxygen octahedra about the cube axes of the high-symmetry cubic (Pm3m) phase. The order parameter for the structural phase transformation is given by $\mathbf{q}=\left\{q_{1}, q_{2}, q_{3}\right\}$ with $q_{1}=q_{2}=0$ and $q_{3}=q \neq 0$. The free energy density for the stress-free monodomain crystal can then be expressed as: 


$$
F_{b u l k}(T, E, q, P)=F_{0}+b_{1} q^{2}+b_{11} q^{4}+a_{1} P^{2}+a_{11} P^{4}-t_{11} P^{2} q^{2}-E P
$$

Here, $b_{1}$ and $b_{11}$ are the structural order parameter susceptibility coefficients, $t_{11}$ is the coupling coefficient between the structural order parameter $q$ and the field-induced polarization $P$, and $a_{1}$ and $a_{11}$ are the dielectric stiffness coefficients.

For $\mathrm{SrTiO}_{3}$, the equilibrium condition for $E=0$ (and thus $P=0$ ) is given by,

$$
\partial F_{b u l k} / \partial q=0
$$

or

$$
q_{S}(T)= \pm \sqrt{-\frac{b_{1}}{2 b_{11}}}
$$

When $E \neq 0$, the condition of thermodynamic equilibrium gives,

$$
\begin{gathered}
\partial F_{b u l k} / \partial q=0 \\
\partial F_{b u l k} / \partial P=E
\end{gathered}
$$

yielding two expressions that must be solved simultaneously,

$$
b_{1} q+2 b_{11} q^{3}-t_{11} P^{2} q=0
$$




$$
2 a_{1} P+4 a_{11} P^{3}-2 t_{11} P q^{2}=E
$$

To describe the effects of mechanical boundary conditions on the structural phase transformation in epitaxial $\mathrm{SrTiO}_{3}$ thin films deposited on a cubic substrate with $(001)_{\text {film }} / /(001)_{\text {substrate }}$, we adopt the particular form of the free energy density given by Pertsev et al. [29,30],

$$
F_{\text {film }}\left(T, E, q, P, u_{m}\right)=G_{0}+\tilde{b}_{1} q^{2}+\tilde{b}_{11} q^{4}+\tilde{a}_{1} P^{2}+\tilde{a}_{11} P^{4}-\tilde{t}_{11} P^{2} q^{2}-E P
$$

in which the re-normalized (structural and dielectric) stiffness and coupling coefficients are,

$$
\begin{gathered}
\tilde{b}_{1}=b_{1}+2\left(\frac{C_{12}}{C_{11}} \lambda_{11}-\lambda_{12}\right) u_{m} \\
\tilde{b}_{11}=b_{11}-\frac{\lambda_{11}^{2}}{2 C_{11}} \\
\tilde{a}_{1}=a_{1}+2\left(\frac{C_{12}}{C_{11}} g_{11}-g_{12}\right) u_{m} \\
\tilde{a}_{11}=a_{11}-\frac{g_{11}^{2}}{2 C_{11}} \\
\tilde{t}_{11}=t_{11}+\frac{g_{11} \lambda_{11}}{C_{11}}
\end{gathered}
$$

Here, $g_{i j}$ are the electrostrictive constants, and $\lambda_{i j}$ are the coupling coefficients between the strain and the structural order parameter in contracted notation. For $E=0$, the shift in the 
ferroelastic phase transformation temperature as a function of $u_{m}$ is given by Eq. (48) and the two-dimensional clamping effect of the substrate is described by Eq. (49). The equilibrium structural order parameter for $E=0$ can be determined from Eq. (42) with the re-normalized

stiffness coefficients $\widetilde{b}_{1}$ and $\widetilde{b}_{11}$. In the presence of an applied electric field $E$, the condition for thermodynamic equilibrium is given by the equations of state $\partial F_{\text {film }} / \partial q=0$ and $\partial F_{f i l m} / \partial P=E$ such that:

$$
\begin{aligned}
& \tilde{b}_{1} q+2 \tilde{b}_{11} q^{3}-\tilde{t}_{11} P^{2} q=0 \\
& 2 \tilde{a}_{1} P+4 \tilde{a}_{11} P^{3}-2 \tilde{t}_{11} P q^{2}=E .
\end{aligned}
$$

\subsubsection{Strain-Induced Ferroelectric Phases}

In Section 4.5.1, we have not taken into account the possibility of the formation of other ferroelastic/ferroelectric phases as discussed theoretically in Ref. [29,30]. We now extend the analysis by considering the formation of other ferroelastic/ferroelectric phases that can be induced by a misfit strain. The free energy density of the film under $u_{m} \neq 0$ by can be expressed as [29,30], 


$$
\begin{aligned}
F_{\text {film }}\left(P_{i}, q_{i}, u_{m}, E_{i}, T\right) & =F_{0}+\tilde{a}_{1}\left(P_{1}^{2}+P_{2}^{2}\right)+\tilde{a}_{3} P_{3}^{2}+\tilde{a}_{11}\left(P_{1}^{4}+P_{2}^{4}\right) \\
& +\tilde{a}_{33} P_{3}^{4}+\tilde{a}_{12} P_{1}^{2} P_{2}^{2}+\tilde{a}_{13}\left(P_{1}^{2}+P_{2}^{2}\right) P_{3}^{2} \\
& +\tilde{b}_{1}\left(q_{1}^{2}+q_{2}^{2}\right)+\tilde{b}_{3} q_{3}^{2}+\tilde{b}_{11}\left(q_{1}^{4}+q_{2}^{4}\right) \\
& +\tilde{b}_{33} q_{3}^{4}+\tilde{b}_{12} q_{1}^{2} q_{2}^{2}+\tilde{b}_{13}\left(q_{1}^{2}+q_{2}^{2}\right) q_{3}^{2} \\
& -\tilde{t}_{11}\left(P_{1}^{2} q_{1}^{2}+P_{2}^{2} q_{2}^{2}\right)-\tilde{t}_{33} P_{3}^{2} q_{3}^{2} \\
& -\tilde{t}_{12}\left(P_{1}^{2} q_{2}^{2}+P_{2}^{2} q_{1}^{2}\right)-\tilde{t}_{13}\left(P_{1}^{2}+P_{2}^{2}\right) q_{3}^{2} \\
& -\tilde{t}_{31} P_{3}^{2}\left(q_{1}^{2}+q_{2}^{2}\right)-t_{44} P_{1} P_{2} q_{1} q_{2} \\
& -\tilde{t}_{44}\left(P_{1} P_{3} q_{1} q_{3}+P_{2} P_{3} q_{2} q_{3}\right) \\
& +\left(C_{11}+C_{12}-2 C_{12}^{2} / C_{11}\right) u_{m}^{2} \\
& -E_{1} P_{1}-E_{2} P_{2}-E_{3} P_{3}
\end{aligned}
$$

The re-normalized coefficients $\tilde{a}_{i}$ and $\tilde{a}_{i j}, \tilde{b}_{i}$ and $\tilde{b}_{i j}$, and $\tilde{t}_{i j}$ entering Eq. (54) are given by [29,30]:

$$
\begin{array}{cc}
\tilde{a}_{1}=a_{1}-\left(g_{11}+g_{12}-2 \frac{C_{12}}{C_{11}} g_{12}\right) u_{m}, & \tilde{a}_{3}=a_{1}+2\left(\frac{C_{12}}{C_{11}} g_{11}-g_{12}\right) u_{m} \\
\tilde{a}_{11}=a_{11}-\frac{g_{12}^{2}}{2 C_{11}}, & \tilde{a}_{33}=a_{11}-\frac{g_{11}^{2}}{2 C_{11}} \\
\tilde{a}_{12}=a_{12}-\frac{g_{12}^{2}}{C_{11}}, & \tilde{a}_{13}=a_{12}-\frac{g_{11} g_{12}}{C_{11}}-\frac{g_{44}^{2}}{2 C_{44}} \\
\tilde{b}_{1}=b_{1}-\left(\lambda_{11}+\lambda_{12}-2 \frac{C_{12}}{C_{11}} \lambda_{12}\right) u_{m}, & \tilde{b}_{3}=b_{1}+2\left(\frac{C_{12}}{C_{11}} \lambda_{11}-\lambda_{12}\right) u_{m} \\
\tilde{b}_{11}=b_{11}-\frac{\lambda_{12}^{2}}{2 C_{11}}, & \tilde{b}_{33}=b_{11}-\frac{\lambda_{11}^{2}}{2 C_{11}} \\
\tilde{b}_{12}=b_{12}-\frac{\lambda_{12}^{2}}{C_{11}}, & \tilde{b}_{13}=b_{12}-\frac{\lambda_{11} \lambda_{12}}{C_{11}}-\frac{\lambda_{44}^{2}}{2 C_{44}}
\end{array}
$$




$$
\begin{array}{ll}
\tilde{t}_{11}=t_{11}+\frac{g_{12} \lambda_{12}}{C_{11}}, & \tilde{t}_{33}=t_{11}+\frac{g_{11} \lambda_{11}}{C_{11}} \\
\tilde{t}_{12}=t_{12}+\frac{g_{12} \lambda_{12}}{C_{11}}, & \tilde{t}_{13}=t_{12}+\frac{g_{12} \lambda_{11}}{C_{11}} \\
\tilde{t}_{31}=t_{12}+\frac{g_{11} \lambda_{12}}{C_{11}}, & \tilde{t}_{44}=t_{44}+\frac{g_{44} \lambda_{44}}{C_{44}}
\end{array}
$$

where $a_{i}$ and $a_{i j}, b_{i}$ and $b_{i j}$, and $t_{i j}$ are the stress-free, monodomain dielectric stiffness coefficients, structural order parameter susceptibility coefficients, and coupling coefficients between the polarization $P_{i}$ and the structural order parameter $q_{i}$, respectively. In Voigt notation, $g_{i j}$ are the electrostrictive constants and $\lambda_{i j}$ are the coupling coefficients between the strain and $q_{i}$.

Using Eqs. (54) and (55), the equations of state $\partial F_{f i l m} / \partial P_{i}=0$ and $\partial F_{f i l m} / \partial q_{i}=0$ at $E_{i}=0$, the equilibrium polarization $P_{i}^{0}\left(T, E_{i}, u_{m}\right)$ and the equilibrium structural parameter $q_{i}^{0}\left(T, E_{i}, u_{m}\right)$ are obtained, and the adiabatic temperature change $\Delta T$ for the ferroelectric phases can be explicitly calculated from the relation [82],

$$
\Delta T\left(T, E_{i}, u_{m}\right)=\sum_{i=1}^{3}\left(-\int_{E_{a}}^{E_{b}} \frac{T}{C_{E}^{0}\left(T, E_{i}, u_{m}\right)}\left(\frac{\partial P_{i}^{0}\left(T, E_{i}, u_{m}\right)}{\partial T}\right)_{E_{i}} d E_{i}\right)
$$

It should be noted that the constitutive model described in sections 4.1-4.5 is expected to be equally applicable to ferroelectric polymer systems. Recently, Li et al. have used the formalism presented here to compute the electrocaloric properties of two 
poly(vinylidenefluoride-trifluoroethylene) [P(VDF-TrFE)] copolymers (65/35 and 55/45 molar percentages) that exhibit first-order and second-order phase transitions, respectively [83]. Their findings closely parallel the results for ceramic polar dielectrics. These results will be discussed in detail below. 


\section{CHAPTER 5}

\section{INFLUENCE OF COMPOSITION, PHASE TRANSITION ORDER, AND MECHANICAL BOUNDARY CONDITION}

In comparing the electrocaloric behaviors of the ferroelectric compounds $\mathrm{BaTiO}_{3}$ (BTO) and $\mathrm{PbTiO}_{3}(\mathrm{PTO}$ ), it is first instructive to consider the nature of the ferroelectric transitions that occur at the Curie points in these two materials. Because both materials exhibit weak first-order transitions that are close to second-order, it is useful to define a parameter that characterizes degree of deviation from a second-order phase change. Following the usual approach of the Landau theory, this parameter is easily recognized as the relative difference between the Curie temperature $T_{C}$ and the temperature $T_{P F}$ where the paraelectric and ferroelectric phases are in thermal equilibrium in the stress-free crystal,

$$
\Delta=\frac{T_{P F}-T_{C}}{T_{C}}=\frac{\alpha_{11}^{2}}{4 \alpha_{0} \alpha_{111} T_{C}}
$$

It follows from Eq. (57) that $\left(T_{P F}-T_{C}\right) \rightarrow 0$ as the quartic dielectric stiffness coefficient $\alpha_{11} \rightarrow 0$, and under this condition, the transition becomes of second-order. The point in the general electric field-temperature-stress phase diagram of a ferroelectric crystal where a line of first-order transitions passes over to a line of second-order transitions is known as a tricritical point. This is the point where two lines of electrical critical points having opposite signs of $E$ intersect the line of second-order phase transitions. Using the Landau coefficients listed in Table 5, a measure of the degree of deviation from this point for 
the stress-free crystal at zero field may be estimated as $\Delta \cong 0.013$ for BTO, while in PTO is about fifty percent larger, $\Delta \cong 0.019$.

Table 5. Landau Coefficients and Thermodynamic Properties of $\mathrm{BaTiO}_{3}$ and $\mathrm{PbTiO}_{3}$ (in SI units, the temperature $T$ in $\mathrm{K}$ ) [23].

\begin{tabular}{lll}
\hline & BTO & PTO \\
\hline$T_{\mathrm{C}}$ & 383 & 752 \\
$\alpha_{1}$ & $3.3(T-383) \times 10^{5}$ & $3.8(T-752) \times 10^{5}$ \\
$\alpha_{11}$ & $3.6(T-448) \times 10^{6}$ & $-7.3 \times 10^{7}$ \\
$\alpha_{111}$ & $6.6 \times 10^{9}$ & $2.6 \times 10^{8}$ \\
$Q_{12}$ & -0.043 & -0.026 \\
$C_{11}$ & $1.76 \times 10^{11}$ & $1.75 \times 10^{11}$ \\
$C_{12}$ & $8.46 \times 10^{10}$ & $7.94 \times 10^{10}$ \\
\hline
\end{tabular}

With these considerations in mind, it is now useful to compare the family of curves $\left.P(T)\right|_{E}$ and $\left.C_{E}(T)\right|_{E}$ for BTO and PTO at different field strengths $E$ under both mechanically free and clamped boundary conditions. The computed results for BTO $(\Delta=0.013)$ are shown in Figure 3. 

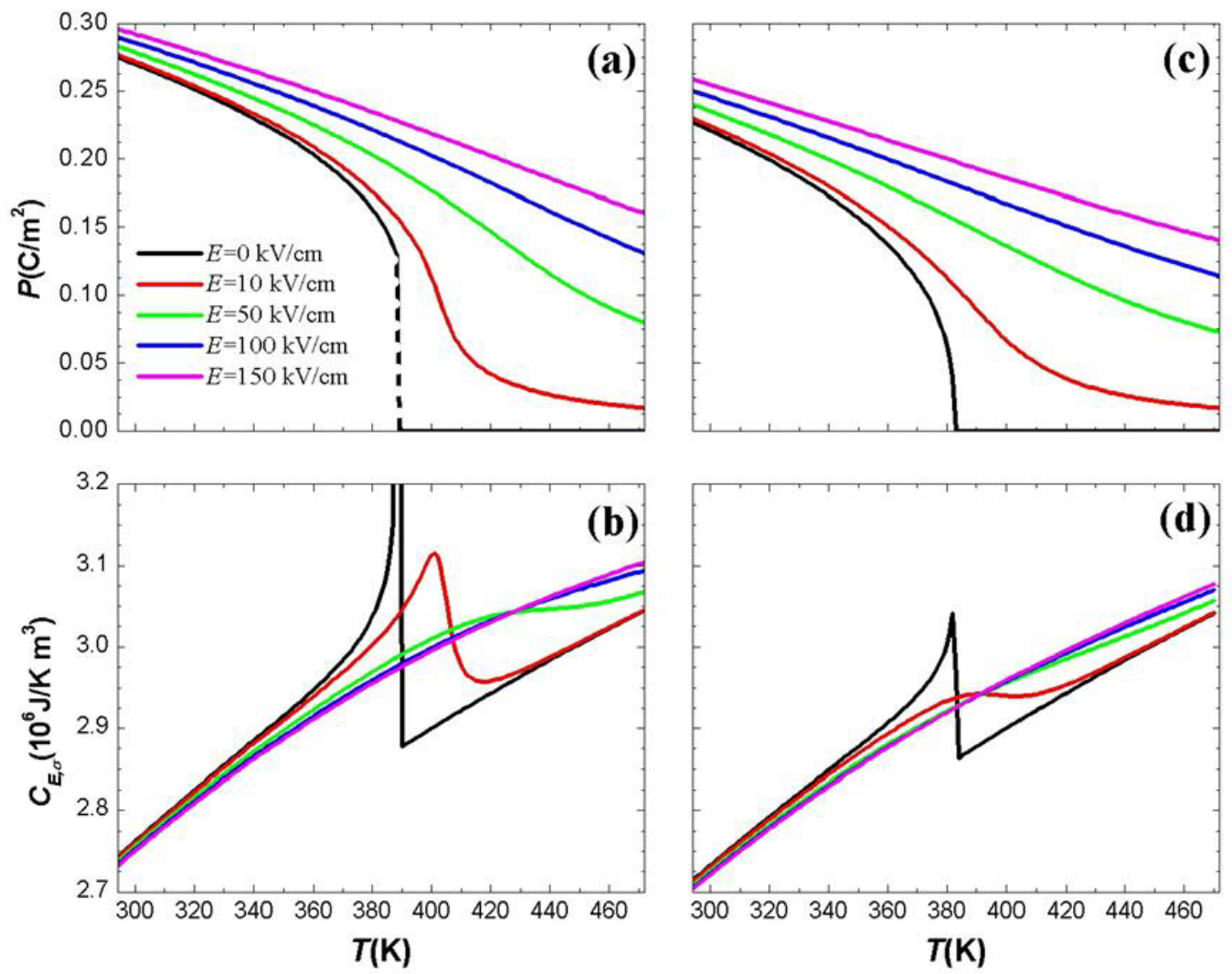

Figure 3. Plots of polarization and specific heat as functions of temperature and applied electric field for monodomain $\mathrm{BaTiO}_{3}$ in the mechanically free state [(a) and (b)], and under prefect lateral clamping [(c) and (d)].

Naturally, the application of the field conjugate $E$ of the order parameter $P$ destroys the discontinuities in thermodynamic properties at the temperature $T_{P F}$ of the first-order paraelectric-ferroelectric phase change. As seen in Figure 3(a), for BTO, a field strength 10 $\mathrm{kV} / \mathrm{cm}$ is sufficiently large to do so. However, the curve $P(\mathrm{~T})$ in this field exhibits an inflection point at $T>T_{P F}$ and the second derivative properties, such as the specific heat $C_{E}$, will therefore exhibit a well-defined maximum, as shown in Figure 3(b). As the field strength is increased, the inflection point will move to higher temperatures, and the gradient of the curve at this point, $\partial P / \partial T$, will diminish. At still higher field strengths the value of $\partial P / \partial T$ at the infection point will be substantially reduced because the majority contribution to the total 
polarization is induced by the electric field. For mechanically-free BTO, a comparison of Figure 3(a) andFigure 3(b) indicates that this behavior occurs for field strengths exceeding $E$ $\sim 100 \mathrm{kV} / \mathrm{cm}$. Under mechanically clamped conditions, the family of curves $\left.P(T)\right|_{E}$ and $\left.C_{E}(T)\right|_{E}$ are qualitatively similar, as shown in Figure 3(c) and (d). However, perfect lateral clamping transforms the zero-field first-order phase transition in to a second-order one, and a much lower field strength $E \sim 50 \mathrm{kV} / \mathrm{cm}$ is required to achieve the same effect as observed at $100 \mathrm{kV} / \mathrm{cm}$ in the stress-free crystal. As can be appreciated by comparing Eq. (24) with Eq. (57), the zero-field first-order transition $\left(\alpha_{11}<0\right)$ is transformed into a second-order one under the condition that the product $Q_{12}^{2} \tilde{C}$ is sufficiently large.

The corresponding family of curves $\left.P(T)\right|_{E}$ and $\left.C_{E}(T)\right|_{E}$ are shown for PTO under mechanically free and clamped boundary conditions in Figure 4. It is clear that owing to the stronger first-order paraelectric-ferroelectric phase change in PTO $(\Delta=0.019)$ the results will differ, but only quantitatively, from those shown for BTO on Figure 3. In this connection there are, however, several points worthy of note. First, despite the stronger first-order phase change in PTO as compared with BTO, the effect of perfect lateral clamping is the same. The product $Q_{12}^{2} \tilde{C}$ is again larger than the absolute value of the quartic dielectric stiffness coefficient $\alpha_{11}$, and the zero-field first-order transition becomes of second-order under mechanical clamping. Second, inspection of Figure 4(a) reveals that, in contrast to the weaker first-order phase change in BTO, a field strength $E=10 \mathrm{kV} / \mathrm{cm}$ is not sufficient to destroy the discontinuities in first derivative thermodynamic properties for the mechanically free crystal. Inspection of Figure 4(a) and (b) shows that at some field $0<E<50 \mathrm{kV} / \mathrm{cm}$ there should exist an electrical critical point, where both $\partial P / \partial T$ and $C_{\mathrm{E}}$ approach infinity. 
Very close to this point the electrocaloric effect will be maximal but also show a strong dependence on temperature over a very narrow range. Finally, it is clear that even at field strengths as large as $E=150 \mathrm{kV} / \mathrm{cm}$, remnants of the temperature variation of the spontaneous polarization due to the phase change persist in both the mechanically free and clamped states to temperatures that are significantly higher than the transition temperatures, $T_{P F}$ and $T_{C}$, respectively. This is evidenced by the weak maxima observed in the specific heat curves, as shown in Figure 4(c) and (d).
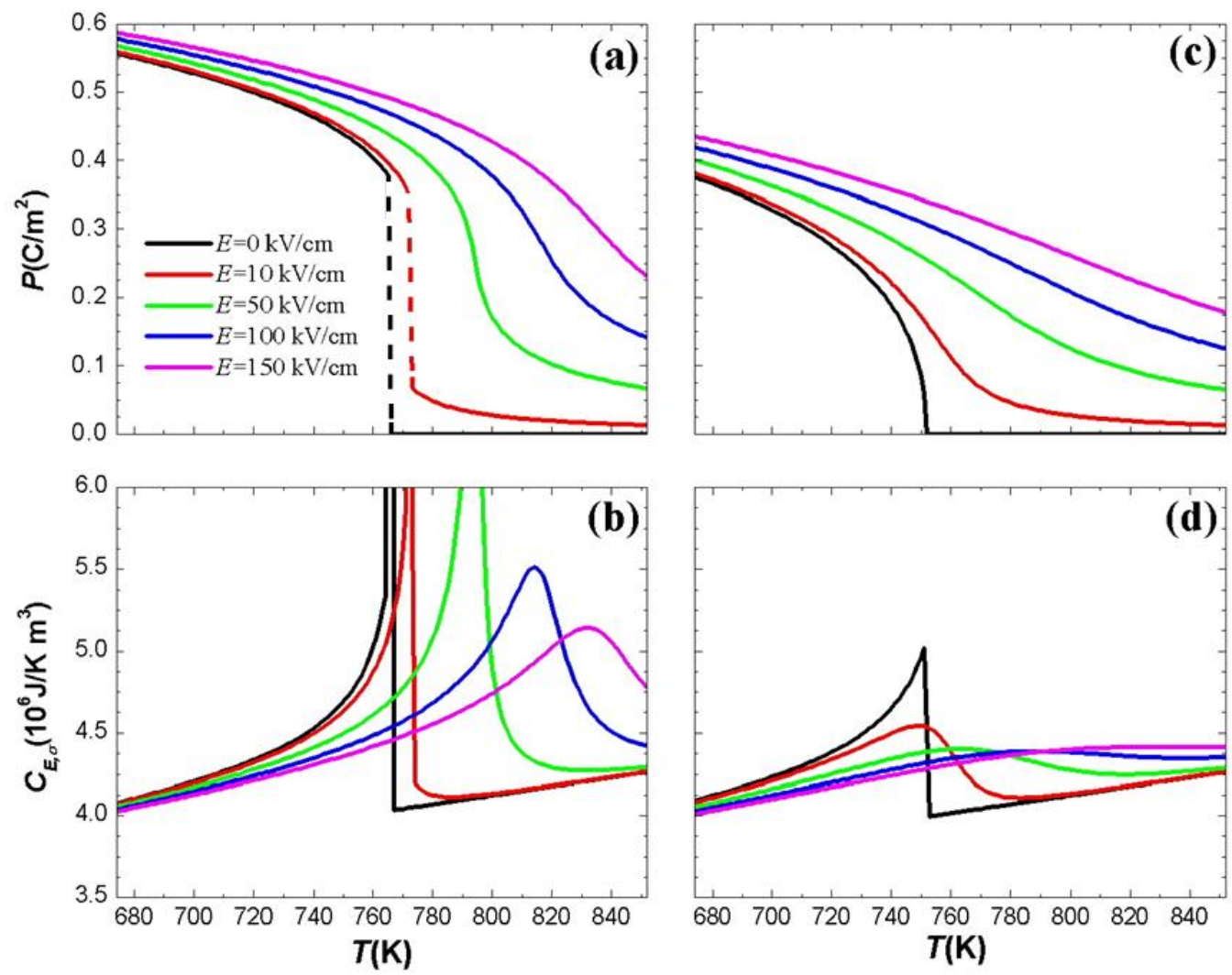

Figure 4. Plots of polarization and specific heat as functions of temperature and applied electric field for monodomain $\mathrm{PbTiO}_{3}$ in the mechanically free state [(a) and (b)], and under prefect lateral clamping [(c) and (d)]. 

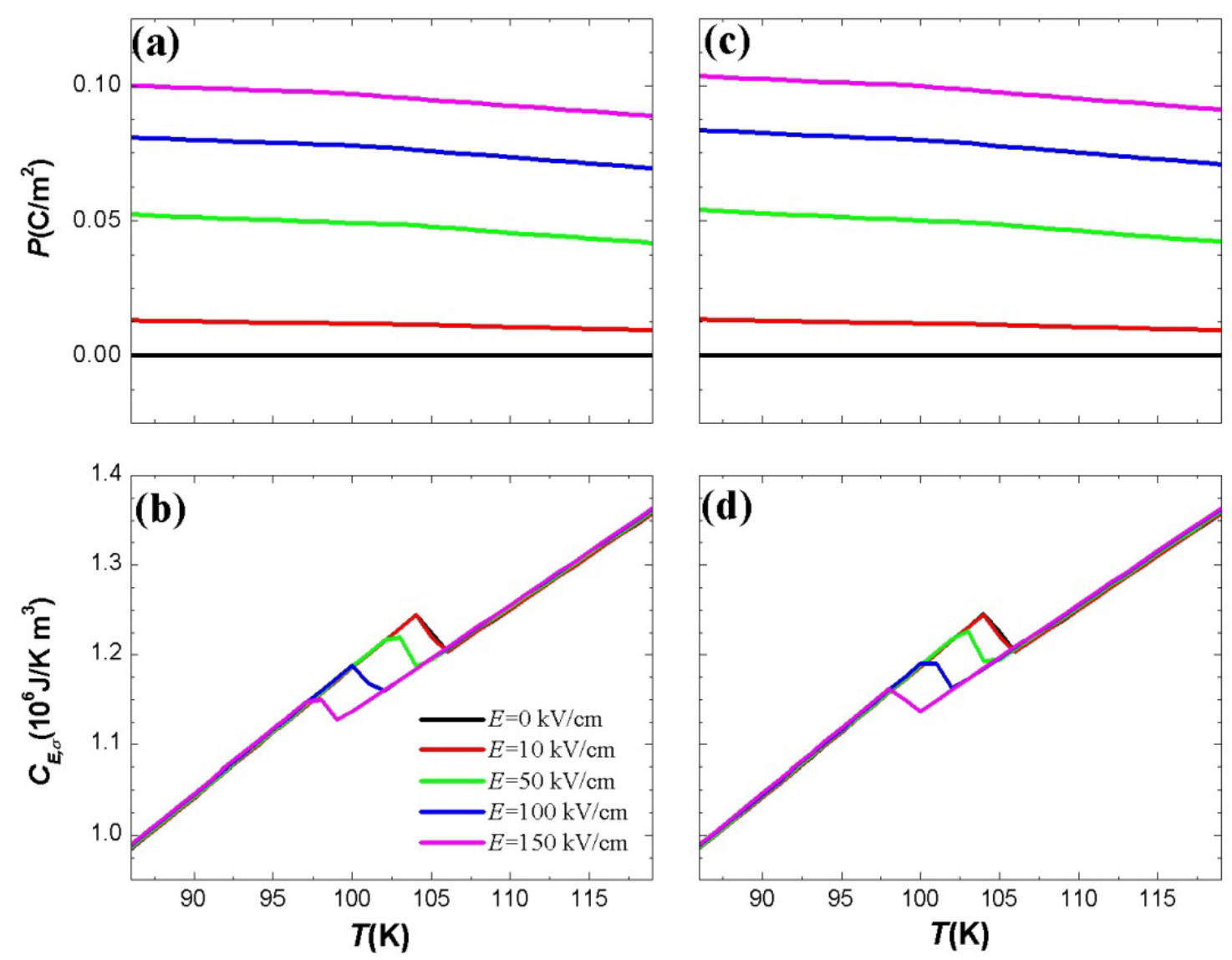

Figure 5. Plots of polarization and specific heat as functions of temperature and applied electric field for monodomain STO in the mechanically free state [(a) and (b)], and under prefect lateral clamping [(c) and (d)].

The effect of an electric field on the ferroelastic phase change in $\mathrm{SrTiO}_{3}$ is, of course, fundamentally different. In STO all of the polarization is induced by the applied field, and as seen in Figure 5(a) and (c), the $\left.P(T)\right|_{E}$ curves change little with changes in temperature and mechanical boundary constraints. In this instance, the main effect of an applied field is to shift the temperature $T_{s t}$ of the second-order ferroelastic phase change to lower temperatures, as can be seen by the behavior of the specific heat shown in Figure 5(b) and (d). Physically 
interpreted, the effect of the electric field is to oppose the rotation of the oxygen octahedra about the (pseudo-cubic) four-fold axis oriented normal to the film surface, stabilizing the higher symmetry cubic phase relative to the lower temperature tetragonal phase. Under different mechanical boundary conditions (e.g., with $u_{m} \neq 0$ ) a non-polar phase could be induced, and this simple picture would be modified.

Recalling that the experimental measurement conditions for the EC effect are defined by the Maxwell relation, $p=(\partial S / \partial E)_{T}$, the influence of the differing phase transition behaviors described above on the electrocaloric responses can best be appreciated by examining the field dependence of the excess entropy along lines of constant temperature. The results computed for BTO under mechanically free and clamped conditions are shown in Figure 6. For the mechanically free crystal it is seen in Figure 6(a) that, on increasing the field from an initial value $E_{a}=0$ to a nonzero field $E_{b}>E_{a}$, the maximum change in entropy will always occur at the temperature of the first-order zero-field ferroelectric phase transition $T=T_{P F}$. This occurs because the majority contribution to the entropy change at this temperature is not due to the electrocaloric effect, but instead arises from the discontinuous change in entropy $S^{\mathrm{XS}}$ at the first-order transition, $\Delta S^{\mathrm{XS}}=L / T_{P F}$ where $L$ is the latent heat. 


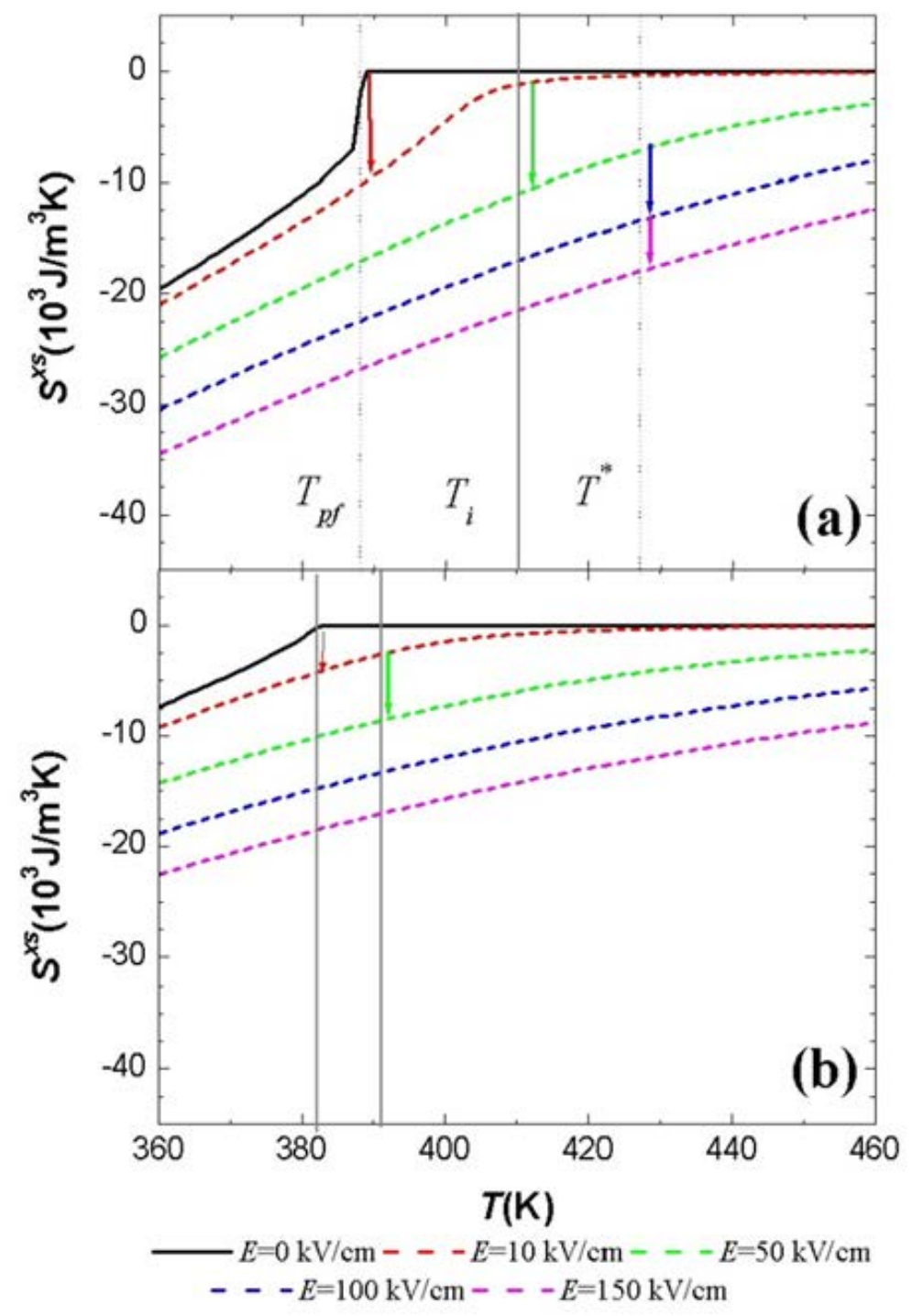

Figure 6. Excess entropy as a function of temperature at different electric fields for (a) mechanically free (bulk) and (b) perfectly laterally clamped $\mathrm{BaTiO}_{3}$. At $E=0$ there is a discontinuity at the paraelectric-ferroelectric transition and as $E$ increases this discontinuity disappears. The vertical lines mark the temperatures where the maximum change in entropy occurs for an electric field change $\Delta E$ having differing values of the bias field $E_{a}$ and final field $E_{b}$ as shown by the arrows (see text for details).

Alternatively, if the field increase is made to take place starting from a much higher field that is sufficient to cause the discontinuities at $T_{P F}$ to disappear as discussed above (e.g., $E^{*}=50 \mathrm{kV} / \mathrm{cm}$ ), the maximum entropy change will always occur at some higher temperature 
$T=T^{*}$. On the other hand, when the field is increased from an intermediate nonzero value, e.g., $E_{a}=10 \mathrm{kV} / \mathrm{cm}<E^{*}$, the maximum entropy change will occur at some different but intermediate temperature $T_{P F}<T_{i}<T^{*}$. Consequently, it is clear that when the electric field is changed between two values $E_{a}$ and $E_{b}$, both the magnitude of the electrocaloric effect and temperature at which it is maximized depend not only on the extent of the field change $\Delta E=$ $E_{b}-E_{a}$ but also on the value of the initial field $E_{a}$. It follows that under mechanically clamped conditions, where the zero-field paraelectric-ferroelectric transition is of second-order, $E^{*}$ may adopt a smaller value compared with that for the mechanically free crystal.

Under experimental conditions, $E_{a}$ is a bias field and the field difference $\Delta E$ is the deviation above this bias. It is an important parameter to be specified, and it depends not only on the mechanical boundary conditions but also on the nature of the phase change in the bulk crystal. That this is the case is evident from Figure 7, where the field dependence of the excess entropy as function of temperature for PTO is plotted. Again, the qualitative results are the same as those for BTO, but owing to the stronger first-order phase change in PTO, they differ quantitatively. For PTO, it is clear that even under clamped conditions, the shapes of the entropy-temperature curves will continue to evolve with electric field, even if a bias field as large as $150 \mathrm{kV} / \mathrm{cm}$ were to be applied. As a result, the maximum in the electrocaloric effect observed for differing values of $\Delta E$ above this bias will be displaced to lines of progressively higher temperatures. Because of the stronger first-order phase change in PTO as compared with BTO, a much larger bias field is required to minimize the dependence of the electrocaloric effect on temperature. For comparison, the entropytemperature curves for STO are shown in Figure 8. For STO, the primary effect of an electric field is to produce a small displacement of these curves toward the origin, relative to the 
zero-field temperature $T_{\text {st }}$ of the second-order ferroelastic transition, as a consequence of the change in the excess specific heat due to the temperature and field dependencies of the structural order parameter.

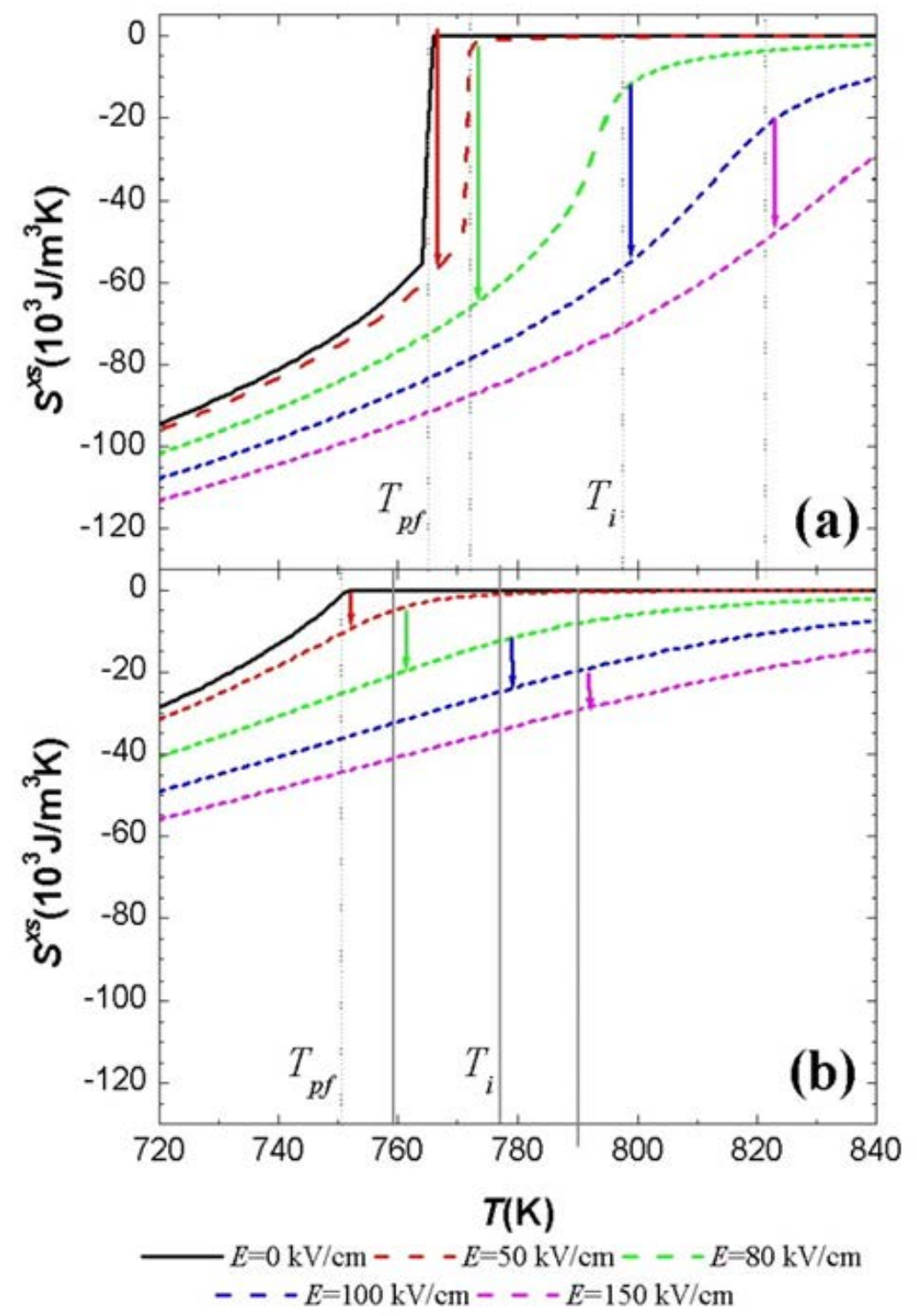

Figure 7. Excess entropy as a function of temperature at different electric fields for (a) mechanically free (bulk) and (b) perfectly laterally clamped $\mathrm{PbTiO}_{3}$. 


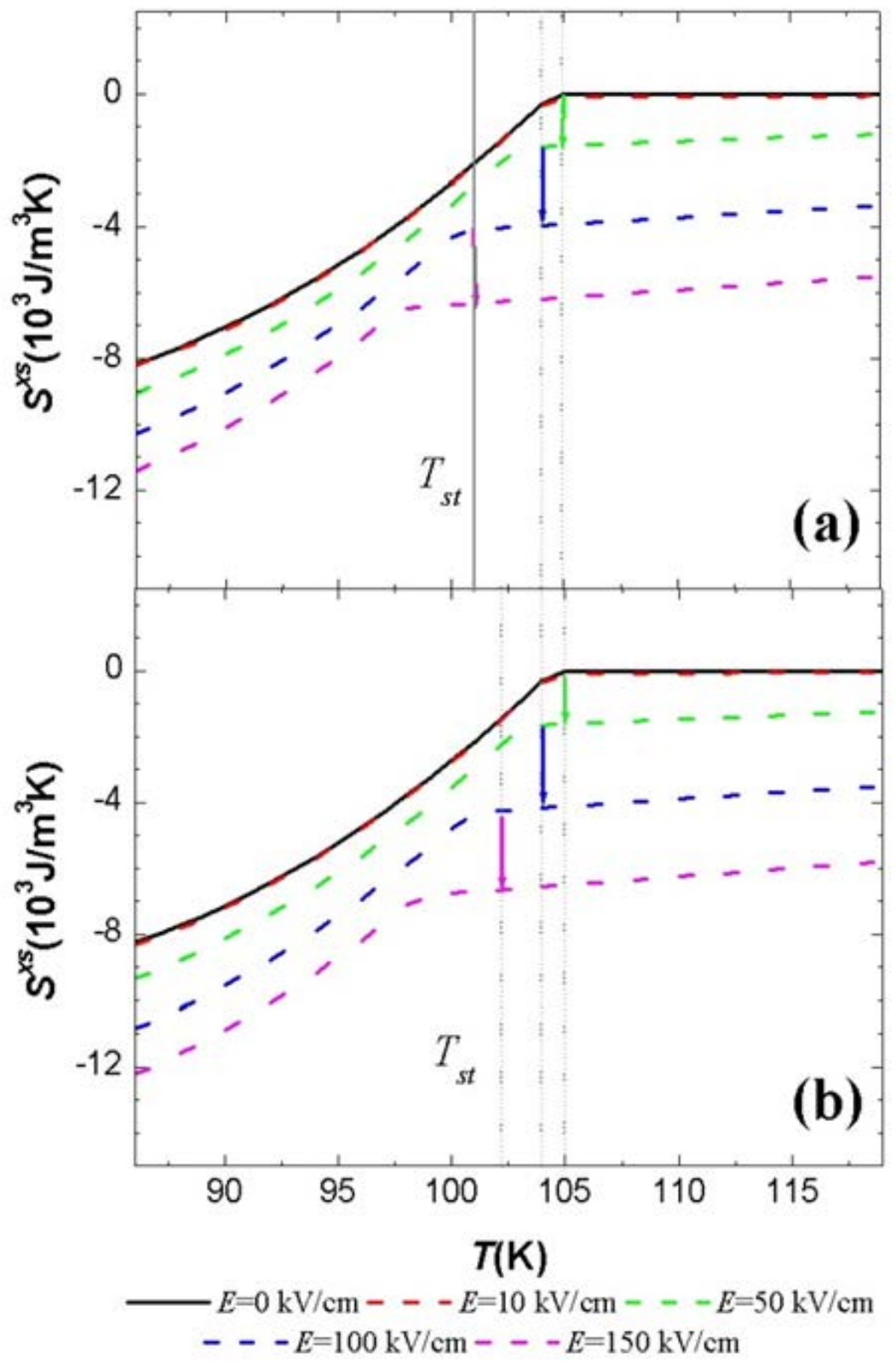

Figure 8. Excess entropy as a function of temperature at different electric fields for (a) mechanically free (bulk) and (b) perfectly laterally clamped $\mathrm{SrTiO}_{3}$ near the structural phase transformation temperature.

The adiabatic temperature changes corresponding to the electrocaloric and electrothermal effects occurring near the ferroelectric and ferroelastic phase transitions in BTO, PTO and STO can be computed using Eq. (9). These are compared in Figure 9 as 
functions of temperature and electric field change, $\Delta E$. In all cases the field change $\Delta E$ is taken relative to a bias field of $E_{a}=50 \mathrm{kV} / \mathrm{cm}$. The results for BTO, PTO and STO under mechanically free conditions are compared in Figure 9(a), (c) and (e) and the corresponding results obtained under perfect lateral clamping are compared in Figure 9(b), (d) and (e), respectively. It is seen that for this choice of bias field the adiabatic temperature changes occurring near the first-order phase change in BTO under mechanically free conditions is modest, with a maximum value of $\Delta T \sim 1.6 \mathrm{~K}$. For the stronger first-order change in PTO the maximum electrocaloric effect is much larger, $\Delta T \sim 9 \mathrm{~K}$, but the effect is far more sensitive to temperature than it is for BTO under the same conditions. In STO, there is no true electrocaloric effect, and the temperature change arising from the change in the excess specific heat near the second-order structural phase transition is very small, $\Delta T<0.5 \mathrm{~K}$. For BTO and PTO mechanical clamping transforms the first-order transitions into second-order ones, and so at the same bias field, both the magnitude and the temperature dependence of the electrocaloric $\Delta T$ are reduced. For the stronger first-order phase change in PTO, it is evident based on the results described above that an increase in the bias field to a value $E_{a}>$ $150 \mathrm{kV} / \mathrm{cm}$ would further reduce the temperature sensitivity. As may be expected, for STO, mechanical clamping has no appreciable effect on the electrothermal temperature change. 

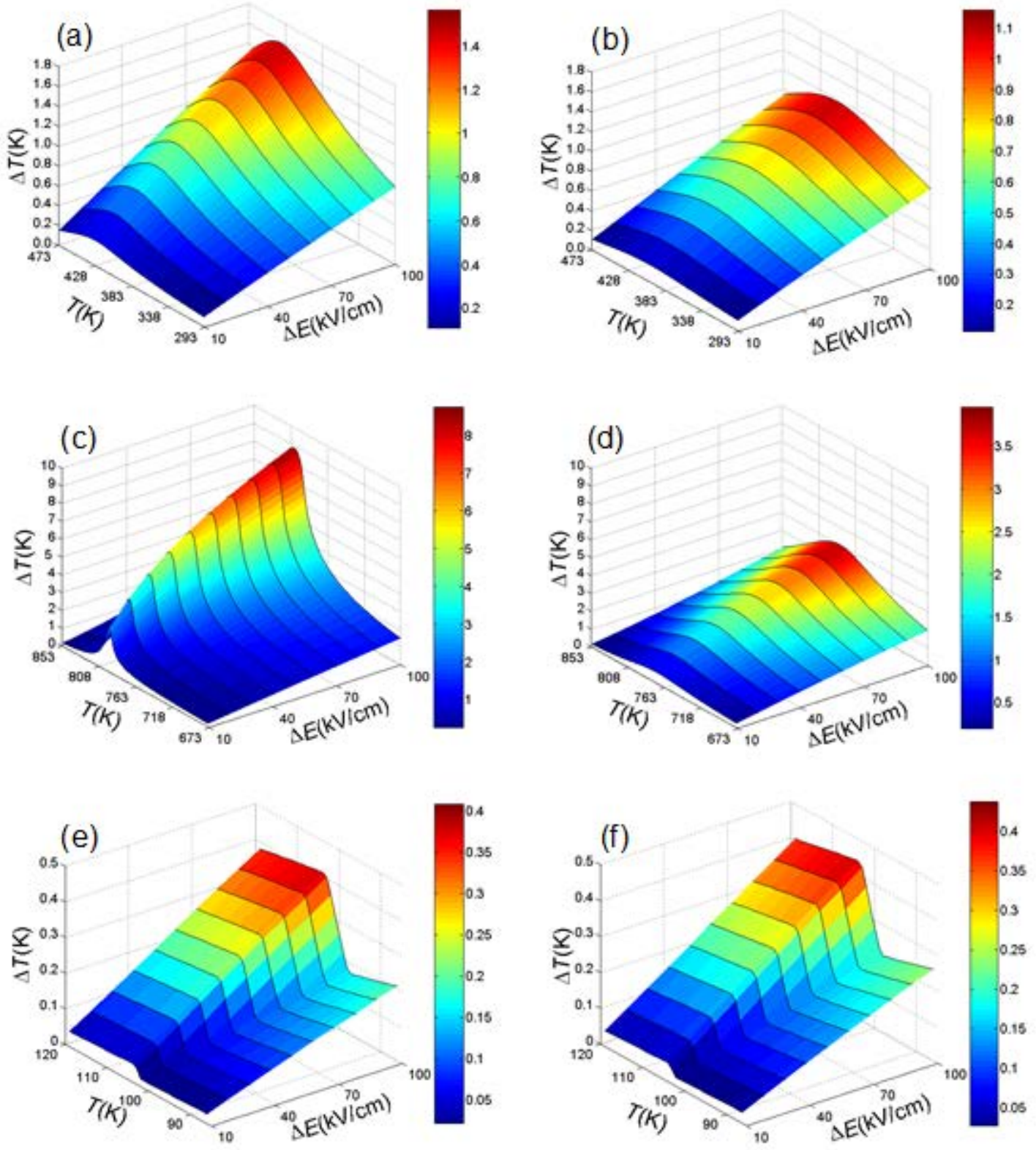

Figure 9. Three-dimensional plots of the adiabatic temperature change $\Delta T$ as functions of $T$ and $\Delta E\left(E_{a}=50 \mathrm{kV} / \mathrm{cm}\right)$ for monodomain uniaxial (a), (c), and (e) unclamped stress-

free (bulk) $\mathrm{BaTiO}_{3}, \mathrm{PbTiO}_{3}$, and $\mathrm{SrTiO}_{3}$, respectively, and (b), (d), and (f) $\mathrm{BaTiO}_{3}$, $\mathrm{PbTiO}_{3}$, and $\mathrm{SrTiO}_{3}$ thin films with $u_{m}=0$, respectively.

The data shown in Figure 9 also provides a qualitative indication concerning how the electrocaloric response of solid solutions comprising BTO, PTO and/or STO as end member compounds may be expected to change with composition under the specific boundary 
conditions investigated. In the pseudo-binary phase diagrams of these systems, both the Curie temperature and the first-order character of the ferroelectric phase transition can be continuously varied with composition. For example, in the pseudo-binary solid solution $\mathrm{BaTiO}_{3}-\mathrm{SrTiO}_{3}$ (BST) the Curie temperature can be decreased from that of BTO ( 383 K) over a temperature range extending down to about $150 \mathrm{~K}$. In this instance, the composition change plays a role analogous to effect of hydrostatic pressure, and the order of the ferroelectric transition continually decreases toward a tricritical point as the strontium concentration is increased. The nature of the phase change, for a given composition (and hence Curie point), may however, also be modified by compressive or tensile misfit strains arising from thermal stresses or lattice mismatch with the substrate. In choosing a ferroelectric material with a Curie temperature appropriate for use a in given temperature range, the electrical and mechanical boundary conditions can be adjusted, in conjunction with composition, in order to alter the nature of the phase change. 


\section{CHAPTER 6}

\section{INFLUENCE OF THERMAL STRESSES}

\subsection{Pyroelectric Response}

Figure 10 plots the in-plane tensile thermal strain as a function of $T_{G}$ for BST 70/30 as an example. For this case, since (on average) the CTEs of the substrates are lower than the CTE of the BST $70 / 30$ film in the temperature range of our analysis $\left(25^{\circ} \mathrm{C} \leq T_{G} \leq 800^{\circ} \mathrm{C}\right)$, the in-plane thermal strains are positive and increase with $T_{G}$. For BST 70/30 on (001) Si and csapphire which have isotropic in-plane CTEs, the thermal strains are equibiaxial. The magnitudes of the in-plane strain on $S i$ and on $c$-sapphire as a function of $T_{G}$ are given by the open squares and triangles in Figure 10, respectively. For films on $a$-sapphire, however, the in-plane thermal strains are anisotropic; the two orthogonal components of the thermal strain are given by the open circles and open triangles in Figure 10 as a function of $T_{G}$. The other three compositions (BST 60/40, BST 80/20, and BST 90/10) show similar behavior: increase in tensile in-plane thermal strains with increasing $T_{G}$ (not included). As large tensile strains suppress ferroelectricity and reduce all functional properties of ferroelectrics [36], a lower processing temperature would be needed for BST films on these particular substrates. This will be discussed in detail with respect to the pyroelectric response. 


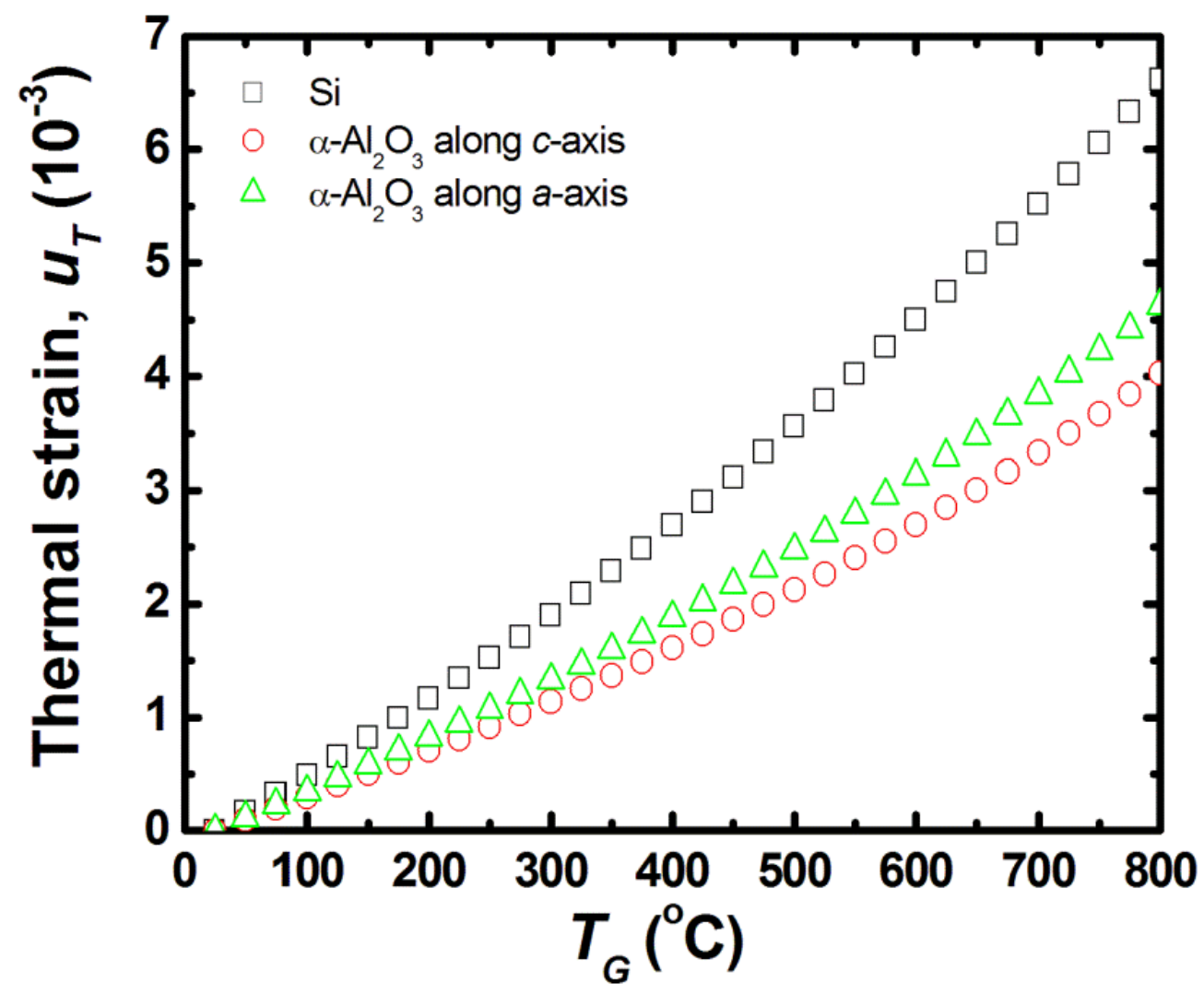

Figure 10. In-plane thermal strain of BST 70/30 films at RT as a function of the growth temperature on $\mathrm{Si}$ and sapphire along $a$ - and $c$ - axes.

The calculated pyroelectric coefficients at RT of the four BST compositions as a function of $T_{G}$ and the applied electric field $(E)$ on the three substrates are shown in Figure 11 [on (001) Si], Figure 12 (on $c$-sapphire), and Figure 13 (on $a$-sapphire) for $25^{\circ} \mathrm{C} \leq T_{G} \leq 800^{\circ} \mathrm{C}$ and $0 \leq E \leq 200 \mathrm{kV} / \mathrm{cm}$. In Figures Figure 11, Figure 12, and Figure 13, plots (a), (b), (c), and (d) correspond to the RT pyroelectric response of BST 60/40, BST 70/30, BST 80/20, and BST 90/10, respectively. As the temperature decreases from $T_{G}$ to RT, thermal strains develop in the film due to the difference of CTEs between the film and the substrate. 

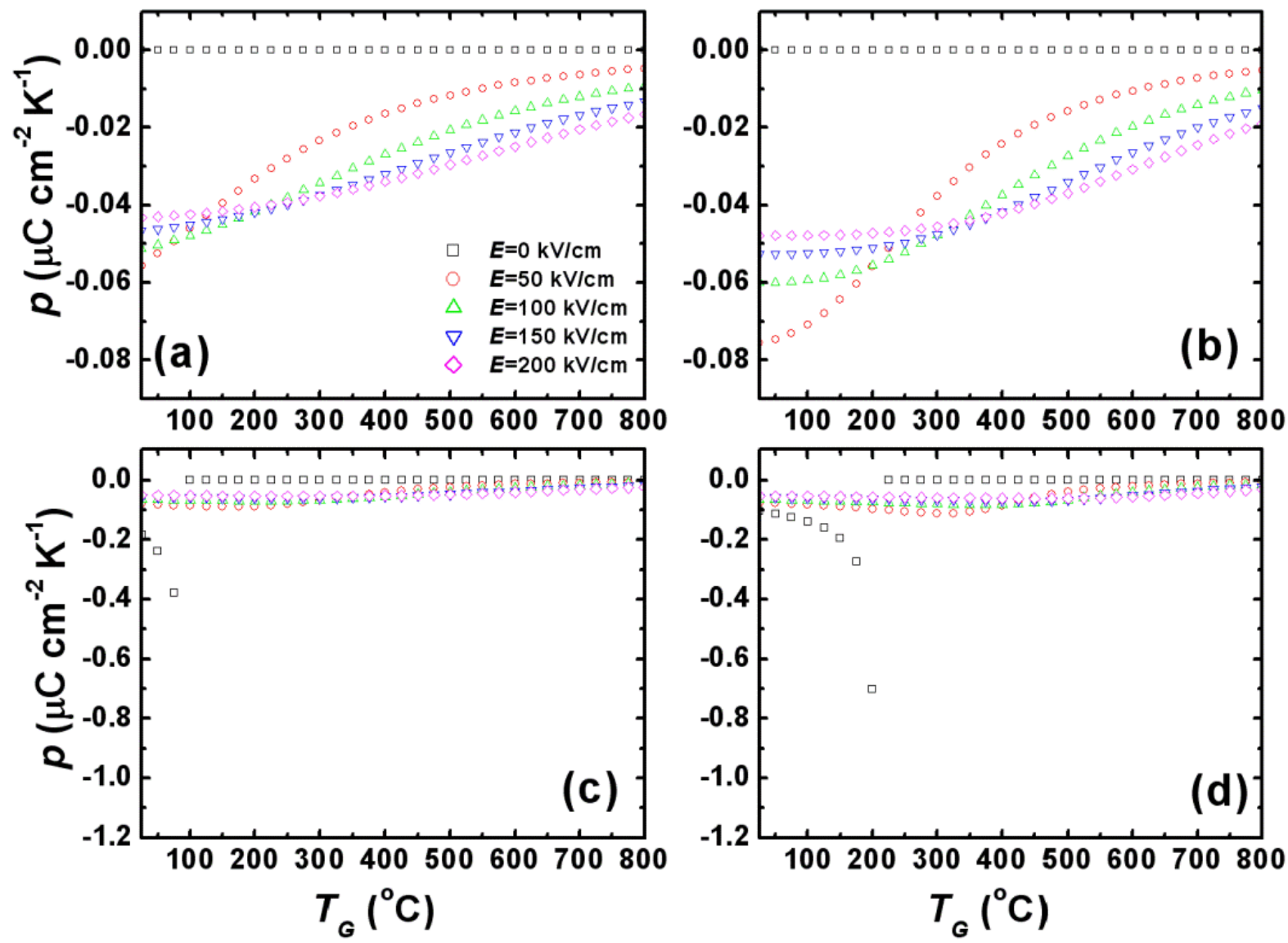

Figure 11. The RT pyroelectric coefficient of BST films with composition (a) BST 60/40 (b) BST 70/30 (c) BST 80/20 (d) BST 90/10 as a function of the growth temperature and the applied electric field on Si substrates.

Table 3 shows that for bulk, un-stressed BST 60/40 and BST 70/30 $T_{\mathrm{C}}$ is below RT $\left(\sim-29^{\circ} \mathrm{C}\right.$ and $8^{\circ} \mathrm{C}$, respectively) while for BST $80 / 20$ and BST 90/10 $T_{\mathrm{C}}$ is above RT $\left(45^{\circ} \mathrm{C}\right.$ and $82^{\circ} \mathrm{C}$, respectively.). In thin film form, $T_{C}$ will shift from its bulk value due to the modification of the quadratic dielectric stiffness coefficient depending on the magnitude of the thermal strain, see Eqs. (32) and (38). In the range of $T_{G}$ that was employed in this analysis, BST 60/40 [Figure 11(a), Figure 12(a), and Figure 13(a)] and BST 70/30 [Figure 11(b), Figure 12(b), and Figure 13(b)] compositions on all three substrates will be in the paraelectric state. As such, the pyroelectric response is entirely due to the polarization 
induced by an applied electric field (hence $p=0$ when $E=0$ ) giving rise to a magnitude of the order of $10^{-2} \mu \mathrm{C} / \mathrm{cm}^{2} \mathrm{~K}$. As the $T_{G}$ increases, i.e., an increase in the in-plane tensile thermal strain, the magnitude of $p$ decreases. In certain $T_{G}$ ranges, $|p|$ does not vary substantially as $E$ is further increased. For instance, for BST 70/30 films on $c$-sapphire [Figure 12(b)], the pyroelectric coefficient remains relatively unchanged for $300^{\circ} \mathrm{C}<T_{G}<$ $500^{\circ} \mathrm{C}$ when $100 \mathrm{kV} / \mathrm{cm}<E<200 \mathrm{kV} / \mathrm{cm}$. For pyroelectric IR detectors in the dielectric bolometer mode, this might be a useful attribute that can produce a pyroelectric response that is relatively insensitive to the processing temperature. It essentially provides a rather large "processing window" and would not require an extremely sensitive temperature control. However, there are two other regions, most prominently seen in Figure 12(b), where there are considerable variations in $|p|$. This is because of the temperature and electric field dependence of the dielectric response [see Eq. (36)]. We will discuss this in detail in connection with the data presented in Figure 14.

For BST 80/20 thin film, $T_{C}=$ RT if $T_{G}=95^{\circ} \mathrm{C}$ on Si [Figure 11(c)], $T_{G}=123^{\circ} \mathrm{C}$ on $c-$ sapphire [Figure 12(c)], and $T_{G}=130^{\circ} \mathrm{C}$ on $a$-sapphire [Figure 13(c)]. These processing temperatures change for the BST 90/10 films; $T_{G}=208^{\circ} \mathrm{C}$ on Si [Figure $\left.11(\mathrm{~d})\right], T_{G}=278^{\circ} \mathrm{C}$ on $c$-sapphire [Figure 12(d)], and $T_{G}=294^{\circ} \mathrm{C}$ on $a$-sapphire [Figure 13(d)]. When $T_{G}$ is lower than these critical values, $T_{C}>\mathrm{RT}$, and the BST films are in the ferroelectric state at RT. Analogous to the cases discussed above for BST 60/40 and 70/30, in the paraelectric state above $T_{\mathrm{C}}, p=0$ when $E=0$. In the ferroelectric state, the pyroelectric response now contains contribution from the temperature dependence of $P_{S}$. This can be considerably larger than the temperature dependence of the induced polarization and increases dramatically as $T_{C}$ is approached. If the thermal strains are engineered in such a way that $T_{C}$ corresponds to RT, 
one can realize pyroelectric coefficients of the order of $1.0 \mu \mathrm{C} / \mathrm{cm}^{2} \mathrm{~K}$ or larger. For example, if a BST 90/10 film on (001) $\mathrm{Si}$ is processed at $T_{G}=208^{\circ} \mathrm{C}$, the $\lambda$-type anomaly associated with the paraelectric-ferroelectric transition occurs at RT and such films would thus have exceptionally high pyroelectric properties. However, the downside of this is that the processing temperature (whether this is the substrate temperature at deposition or annealing temperature) has to be controlled extremely precisely (within $1^{\circ} \mathrm{C}$ ).
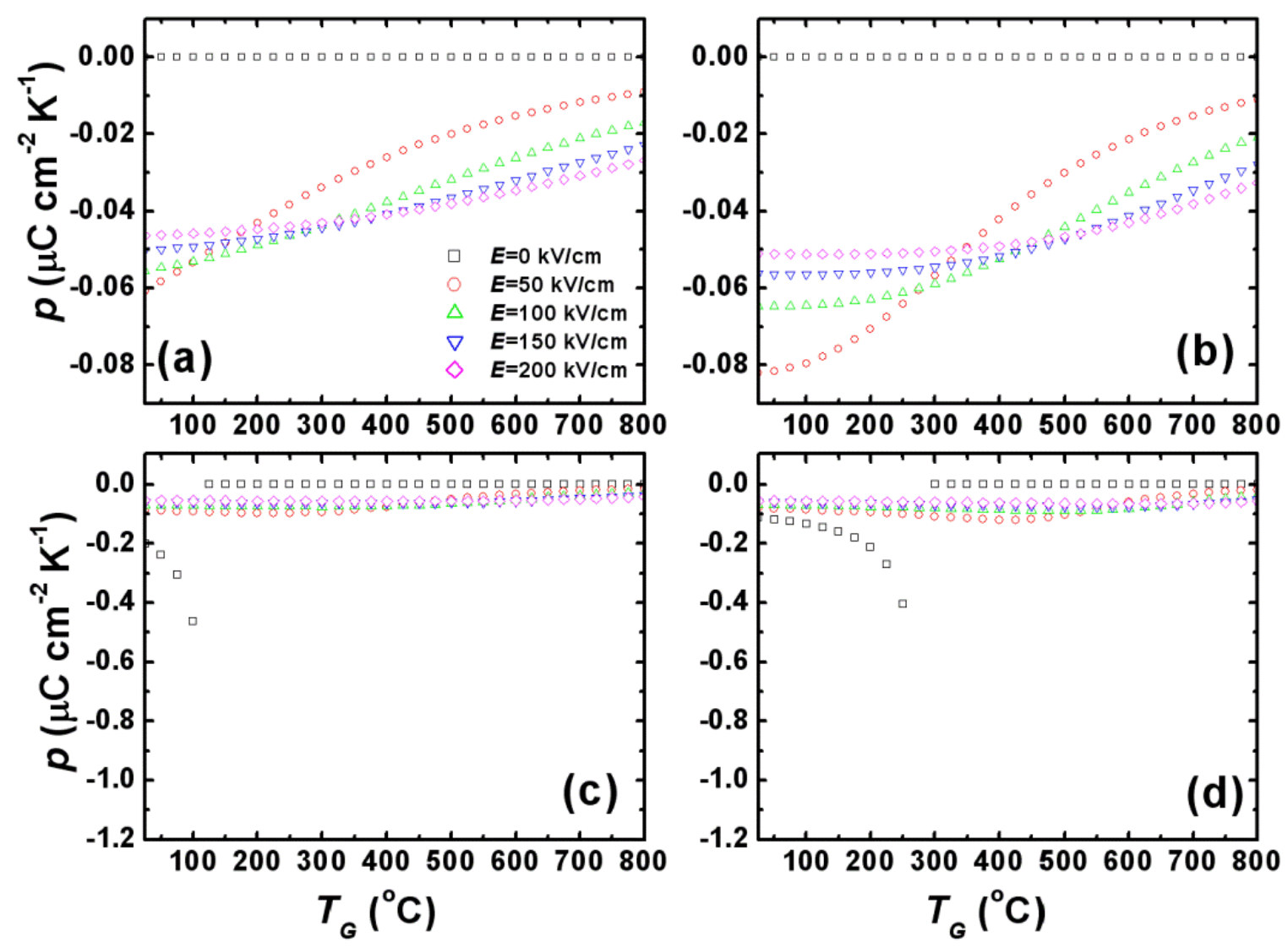

Figure 12. The RT pyroelectric coefficient of BST films with composition (a) BST 60/40 (b) BST 70/30 (c) BST 80/20 (d) BST 90/10 as a function of the growth temperature and the applied electric field on $c$-sapphire substrates. 

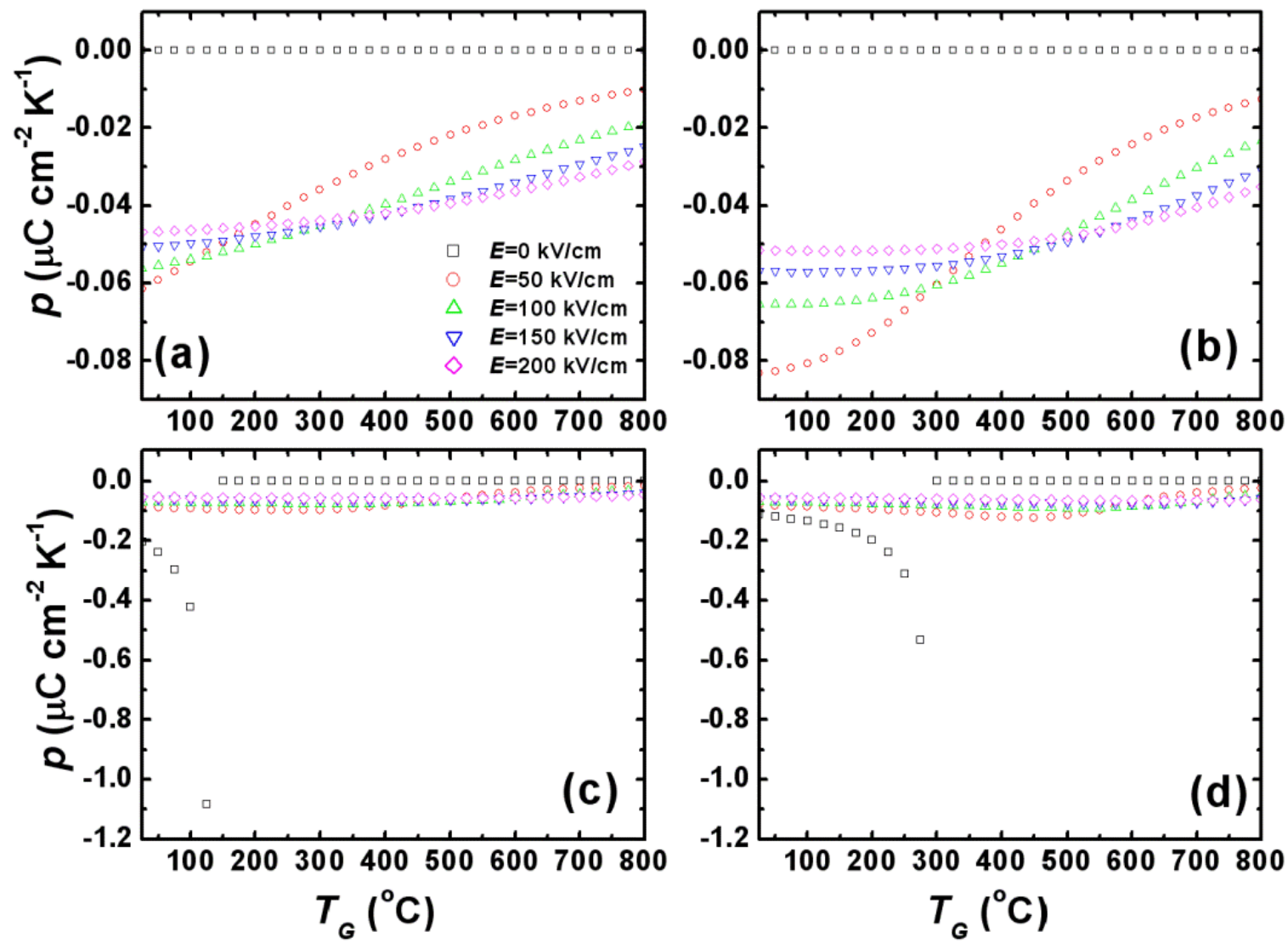

Figure 13. The RT pyroelectric coefficient of BST films with composition (a) BST 60/40 (b) BST 70/30 (c) BST 80/20 (d) BST 90/10 as a function of the growth temperature and the applied electric field on $a$-sapphire substrates.

To discuss the pyroelectric response in the paraelectric in more detail, we present in Figure 14(a) the induced polarization in BST 70/30 on $c$-sapphire as a function of $T_{G}$. Since the film is in paraelectric state, there is no polarization when $E=0$. At a given $T_{G}$, the induced polarization increases with $E$, and at a given $E$, higher induced polarizations are possible for smaller in-plane thermal strains resulting from a lower $T_{G}$. This is also shown in Figure 14(b) where polarization is plotted as a function of $E$ for $T_{G}=100^{\circ} \mathrm{C}$ and $700^{\circ} \mathrm{C}$. 

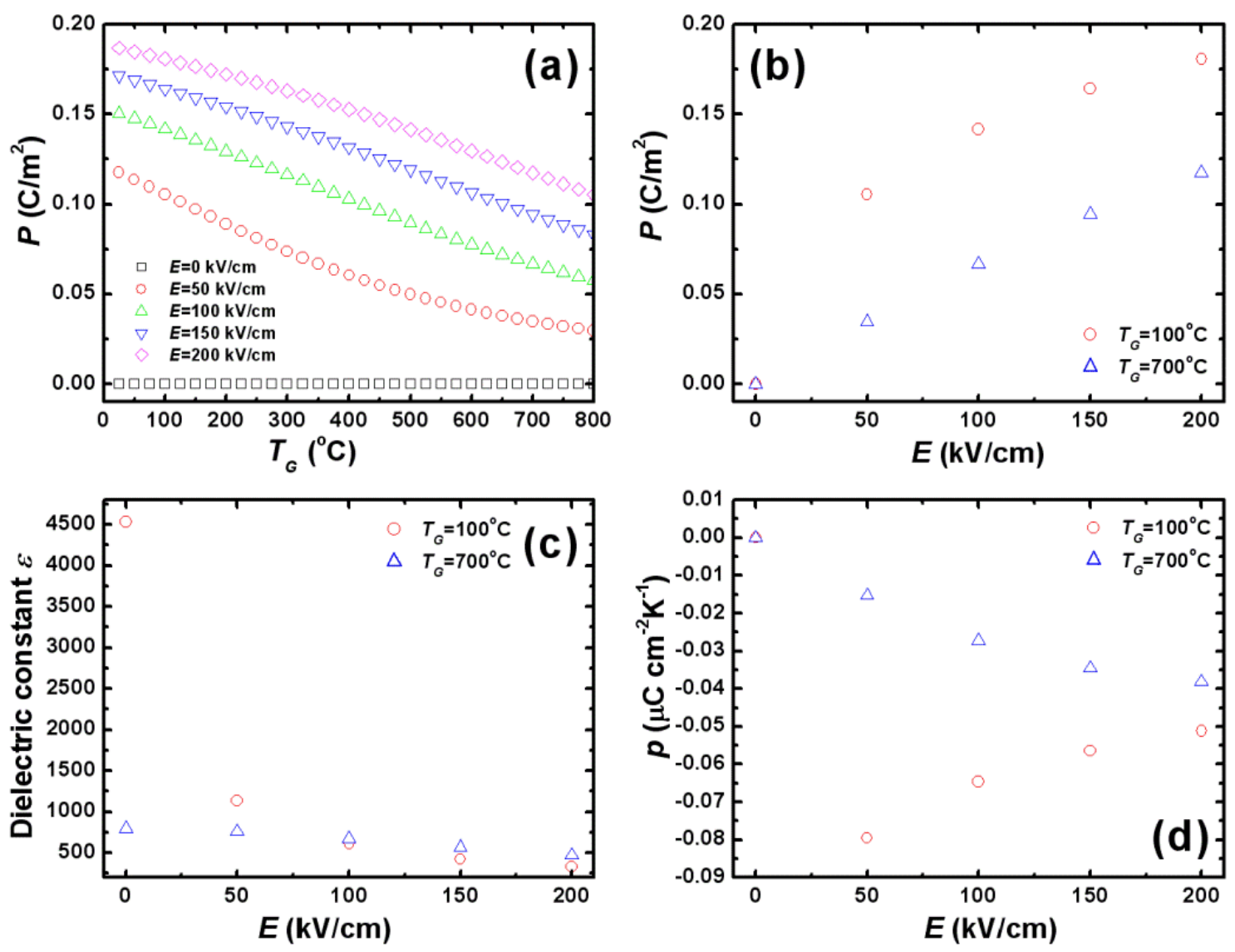

Figure 14. BST 70/30 on c-sapphire at RT: (a) The total polarization as a function of the growth temperature at various applied electric fields; (b) the total polarization for $\boldsymbol{T}_{G}=$ $100^{\circ} \mathrm{C}(\circ)$ and $T_{G}=700^{\circ} \mathrm{C}(\Delta)$ as a function of $E$; (c) the relative dielectric constant for $T_{G}=100^{\circ} \mathrm{C}(\circ)$ and $T_{G}=700^{\circ} \mathrm{C}(\Delta)$ as a function of $E$; and (d) the pyroelectric coefficient for $T_{G}=100^{\circ} \mathrm{C}(\circ)$ and $T_{G}=700^{\circ} \mathrm{C}(\Delta)$ as a function of $E$.

Figure 14(c) displays the variation in the dielectric constant of BST 70/30 on csapphire as a function of $E$ for $T_{G}=100^{\circ} \mathrm{C}$ and $700^{\circ} \mathrm{C}$. For $T_{G}=100^{\circ} \mathrm{C}$, the relative dielectric constant is extremely high at $E=0(4,530)$ and decreases nonlinearly with $E$ to 330 at 200 $\mathrm{kV} / \mathrm{cm}$; a common property of ferroelectrics. The relatively large value of $\varepsilon$ at $E=0$ indicates that while the material is in paraelectric state, $T_{C}$ is not far off $\left(-14^{\circ} \mathrm{C}\right)$ compared to the condition of $T_{G}=700^{\circ} \mathrm{C}$ for which $\varepsilon=800$ at $E=0$ and $T_{C}$ is well below RT $\left(-196^{\circ} \mathrm{C}\right)$. The RT pyroelectric response in the paraelectric state [Figure 14(d)] hence results from the 
complicated interplay of the electric field and thermal strain dependence of the dielectric constant and the $T_{G}$-dependence of the thermal strains. As such, the pyroelectric coefficient in the paraelectric state can be re-written as

$$
p\left(E, u_{T}\right)=\int_{0}^{E}\left(\frac{\partial \varepsilon\left(E, u_{T}\right)}{\partial T}\right)_{E} d E
$$

for the case of isotropic equibiaxial strain $\left[p\left(E, u_{T 1}, u_{T 2}\right)\right.$ for anisotropic substrates]. This leads to the behavior given in Figure 14(d) where $|p|$ decreases for $T_{G}=100^{\circ} \mathrm{C}$ when $E$ is increased but it rises with $E$ for the case of $T_{G}=700^{\circ} \mathrm{C}$.

\subsection{Electrocaloric Response}

The dependence of the thermal strains on the growth temperature for BTO, BST60/40, and PTO on the various substrates is shown in Figure 15. Because the polycrystalline averages of the CTEs for the Si and sapphire substrates are lower than the TECs of the ferroelectric films in the temperature range of our analysis ( $\mathrm{RT} \leq T_{G} \leq 800^{\circ} \mathrm{C}$ ), the in-plane thermal strains are tensile. As seen in Figure 15, their magnitudes increase with $T_{G}$. A principal effect of the tensile strains is to shift the zero-field values of $T_{\mathrm{C}}$ to lower temperatures. This shift in $T_{C}$ arises due to the modification of the quadratic dielectric stiffness coefficient, depending on the magnitude of the thermal strain [Eqs. (32) and (38)]. 

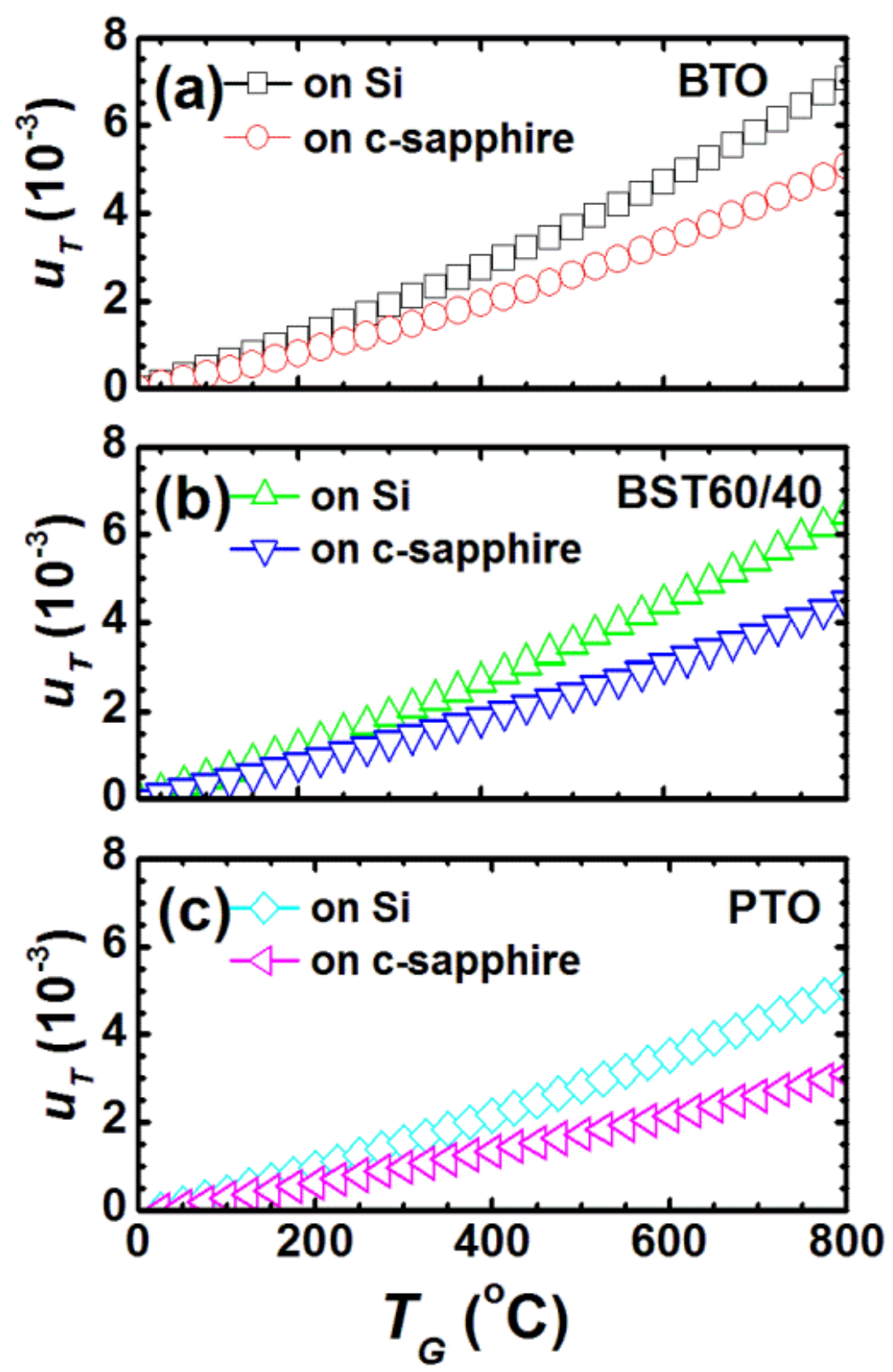

Figure 15. The variation in $u_{T}$ as a function of $T_{G}$ for a) BTO, b) BST 60/40, and c) PTO.

As shown in Figure 16, the thermal strains produce a decrease in the zero-field value of $T_{C}$ of BTO, BST 60/40, and PTO on Si and $c$-sapphire when compared to their bulk values. Naturally, under an applied electric field, the ferroelectric phase change at $T_{\mathrm{C}}$ is 
destroyed and the polarization vs. the electric field curve exhibits an inflection point, approaching zero asymptotically with increasing field. As a consequence, the adiabatic $\Delta T$ and other properties that depend on the derivative $\partial P / \partial T$ will show their maximum values at a temperature $T_{M}>T_{\mathrm{C}}$, slightly above the zero-field values shown in the figure [46].

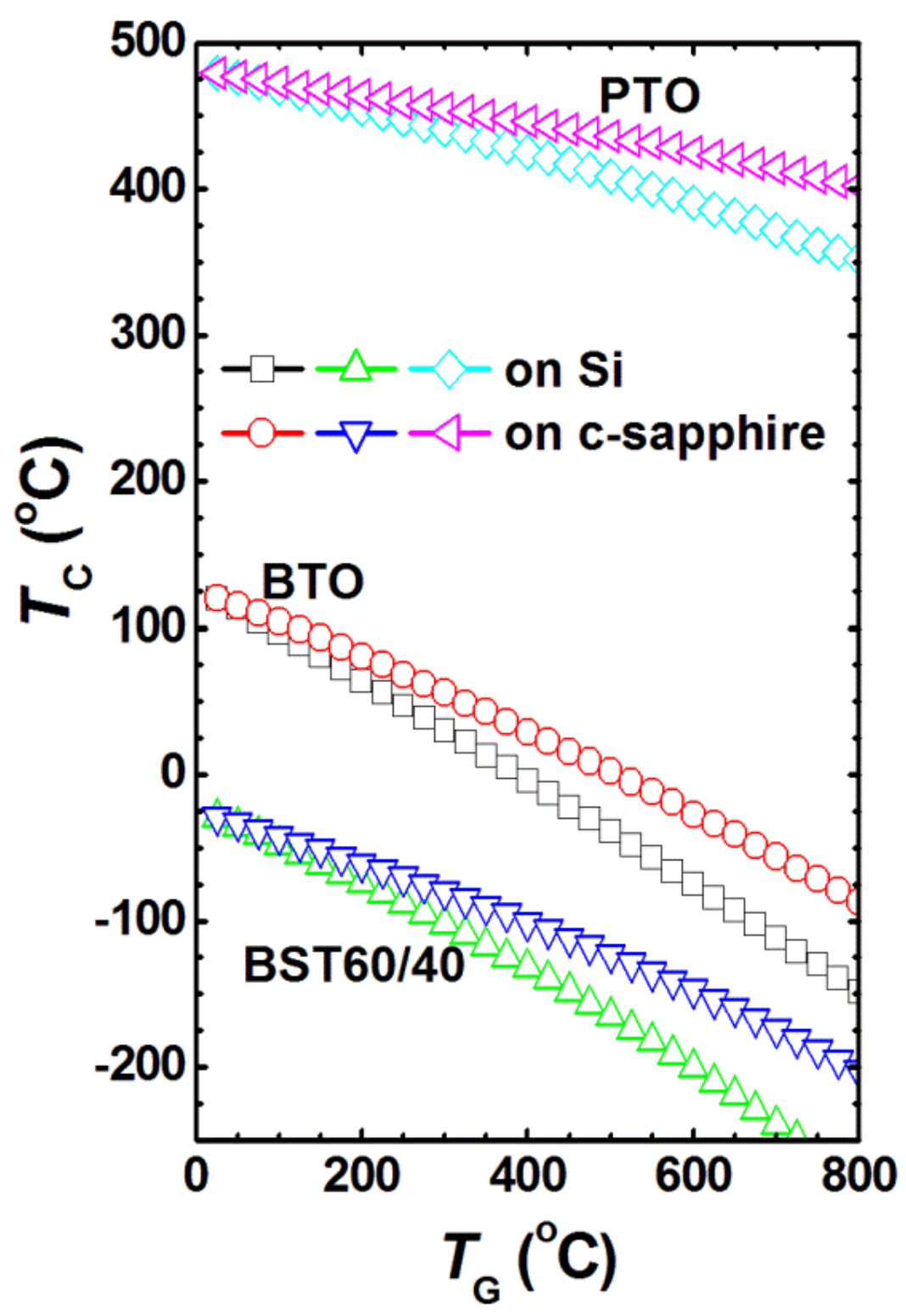

Figure 16. The dependence of $T_{C}$ on $T_{G}$ for BTO, BST 60/40, and PTO on Si and $c$ sapphire. 

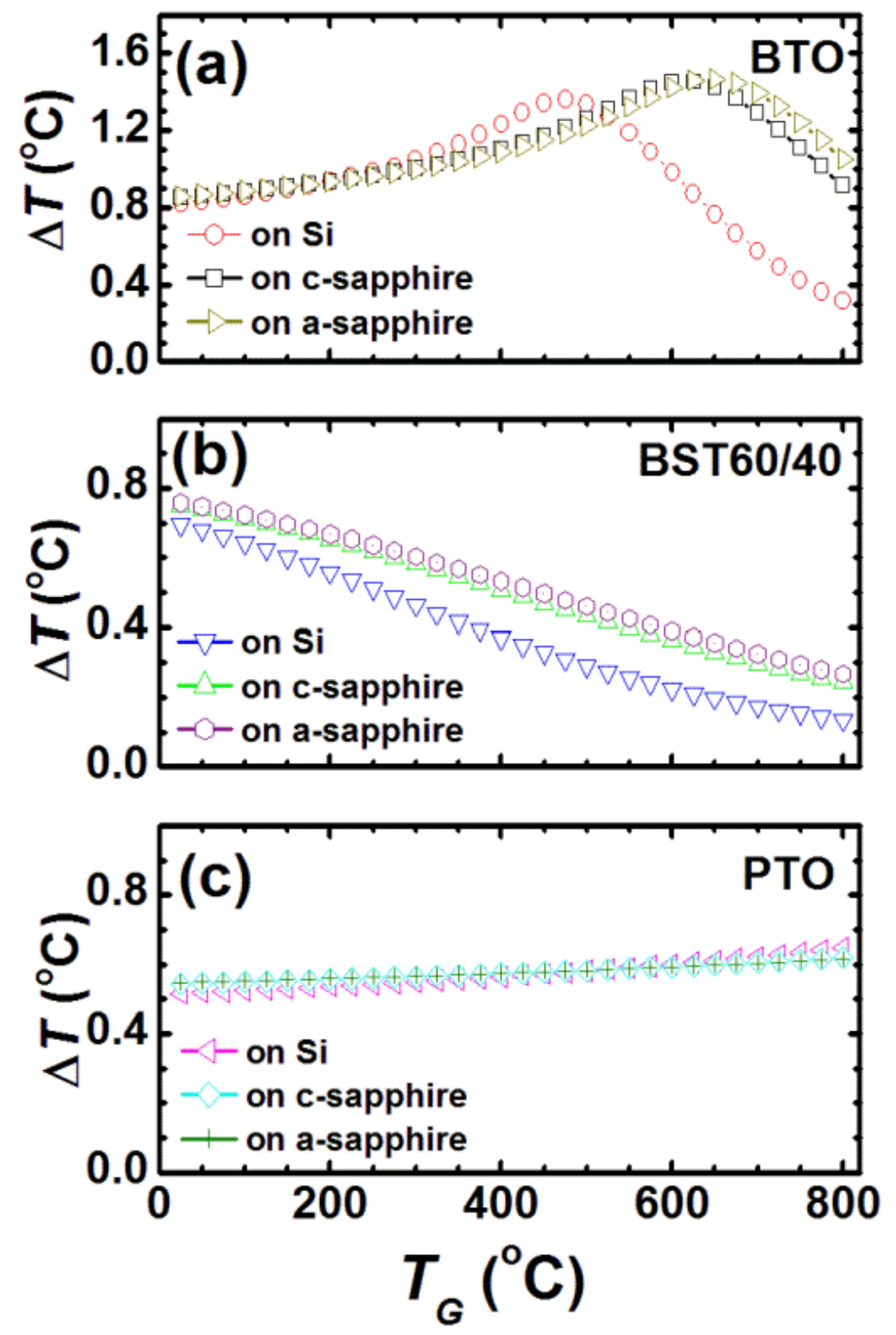

Figure 17. Adiabatic temperature change in (001) textured polycrystalline a) BTO, b) BST 60/40 and c) PTO thin films $\left(E_{a}=50 \mathrm{kV} / \mathrm{cm}, \Delta E=120 \mathrm{kV} / \mathrm{cm}\right)$ as a function of $T_{G}$ on $\mathrm{Si}, c$-sapphire, and $a$-sapphire at RT.

Figure 17 plots the RT values of the adiabatic $\Delta T$ for BTO, BST60/40, and PTO on the various substrates. For the BTO films, the thermal stresses are sufficient to shift the zero- 
field $T_{C}$ to RT. As a result, the electrocaloric response shows a pronounced dependence on $T_{G}$. For BTO on $\mathrm{Si}, c$-sapphire, and $a$-sapphire, a maximum in the electrocaloric response can be produced for growth temperatures $T_{G}$ equal to $\sim 475^{\circ} \mathrm{C}, 625^{\circ} \mathrm{C}$, and $650^{\circ} \mathrm{C}$, respectively. For comparison, results for the BST 60/40 and PTO films are also shown in Figure 17. For BST 60/40 films, $T_{C}$ always lies below RT (Figure 16). As a result, the EC response simply decreases with an increase in $T_{\mathrm{G}}$. In contrast, for the PTO films $T_{C}$ lies far above RT and the adiabatic $\Delta T$ shows only a slight increase with $T_{\mathrm{G}}$. In BST $60 / 40$ and PTO films, the contribution of the phase transition near $T_{\mathrm{C}}$ is avoided; as such, the electrocaloric response is only weakly dependent on $T_{G}$. These results clearly illustrate that thermal stresses can be engineered in such a way that as to shift the maximum in electrocaloric properties to a desired working temperature.

The influence of thermal stresses on the electrocaloric properties of a BTO cooling device working near room temperature can be better appreciated by comparing the adiabatic $\Delta T$ vs. temperature curves for polycrystalline thin film BTO and stress-free monocrystalline bulk BTO, as shown in Figure 18. We note that the temperature dependence of the thermal strain is included in our calculations, i.e., we calculate $u_{T}\left(T, T_{G}\right)$. For BTO films on $\mathrm{Si}$, the thermal stresses for films grown at $450^{\circ} \mathrm{C}$ are sufficient to shift the zero-field Curie temperature $T_{\mathrm{C}}$ to near $\mathrm{RT}$, resulting in a strong enhancement of the electrocaloric properties as compared to the bulk material. It is further seen in Figure 18 that the adiabatic temperature change occurring at RT closely approaches that of the bulk near its Curie temperature. Similar results are obtained for BTO on sapphire substrates. The curves in Figure 18 demonstrate that the growth temperature of polycrystalline ferroelectric films is an important parameter to be controlled in optimizing the electrocaloric properties. 


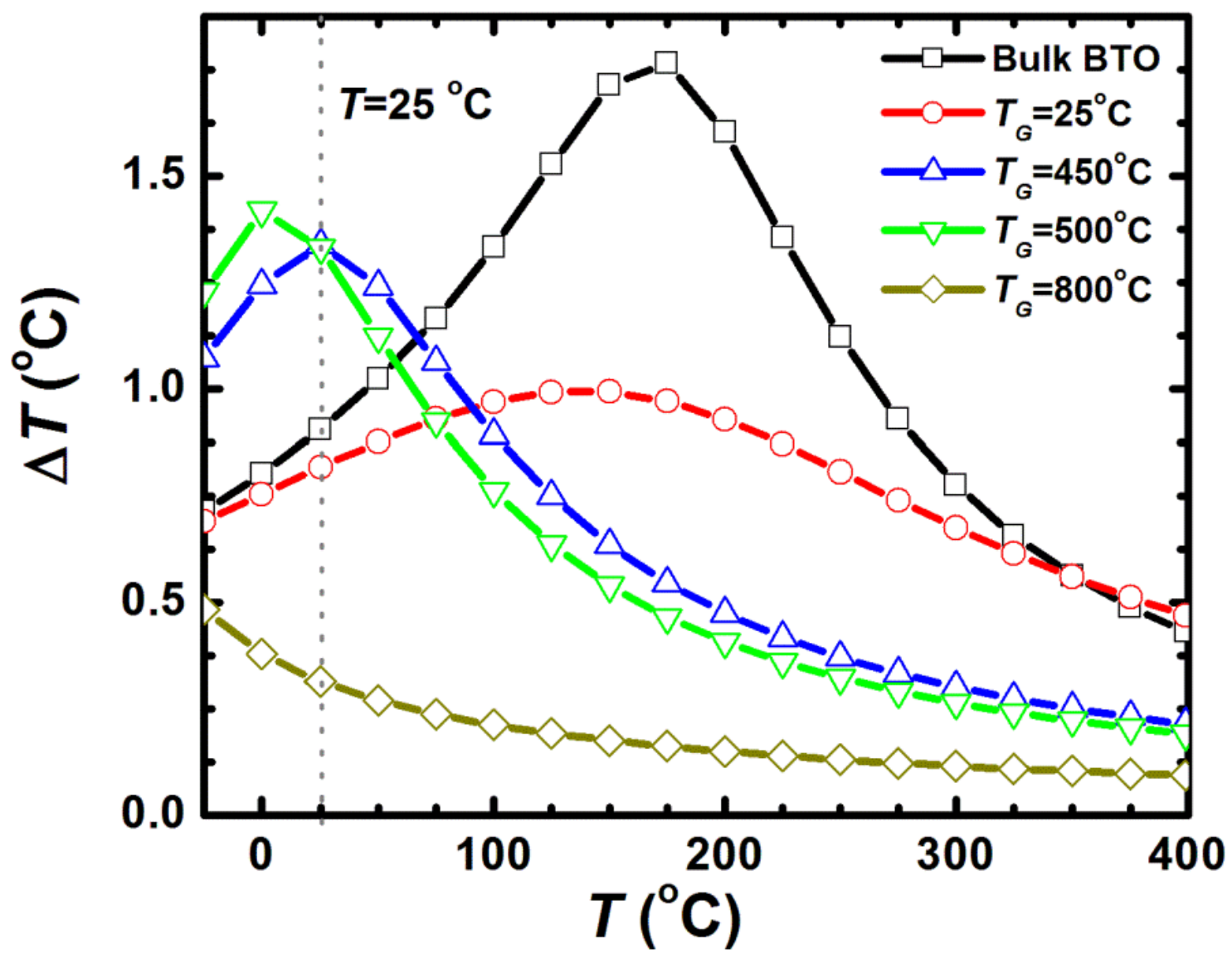

Figure 18. Adiabatic temperature change in Bulk BTO and (001) textured polycrystalline BTO on $\mathrm{Si}\left(E_{a}=50 \mathrm{kV} / \mathrm{cm}, \Delta E=120 \mathrm{kV} / \mathrm{cm}\right)$ as a function of $T$ at different $T_{G}$. 


\section{CHAPTER 7}

\section{INCIPIENT FERROELECTRIC MATERIALS}

Using Eqs. (54) and (55), the equations of state $\partial G / \partial P_{i}=0$ and $\partial G / \partial q_{i}=0$ at $E_{i}=0$, and the values of the property coefficients for STO given in Ref. [29], we obtain the identical misfit $u_{m}-T$ phase diagram of epitaxial monodomain STO films as given by Pertsev et al. [30]. Figure 19 shows the stability regions of various phases for $150 \mathrm{~K}<T<400 \mathrm{~K}$ and $0.02<u_{m}<0.02$. The possible phases and their corresponding order parameters that appear in Figure 19(a) and (b) are: HT: $P_{1}=P_{2}=P_{3}=0, q_{1}=q_{2}=q_{3}=0$; ST: $P_{1}=P_{2}=P_{3}=0, q_{1}=q_{2}=0, q_{3} \neq 0$; FTI: $P_{1}=P_{2}=0, P_{3} \neq 0, q_{1}=q_{2}=q_{3}=0$; FTII: $P_{1}=P_{2}=0, P_{3} \neq 0, q_{1}=q_{2}=0, q_{3} \neq 0$; FOI: $\left|P_{1}\right|=\left|P_{2}\right| \neq 0$, $P_{3}=0, q_{1}=q_{2}=q_{3}=0$. We limit ourselves to these ranges of $T$ and $u_{m}$ since lower operating temperatures are not of great interest for electrocaloric cooling devices and misfit strains larger than $2 \%$ in magnitude (depending on the substrate material and film thickness) would be partially or completely relaxed via the formation of two-dimensional periodic arrays of interfacial dislocations [84]. The effect of misfit dislocations can certainly be incorporated into the model using an "effective" substrate lattice parameter [85], but this would unnecessarily complicate the physical interpretation of the results and would obscure the effect of $u_{m}$. 

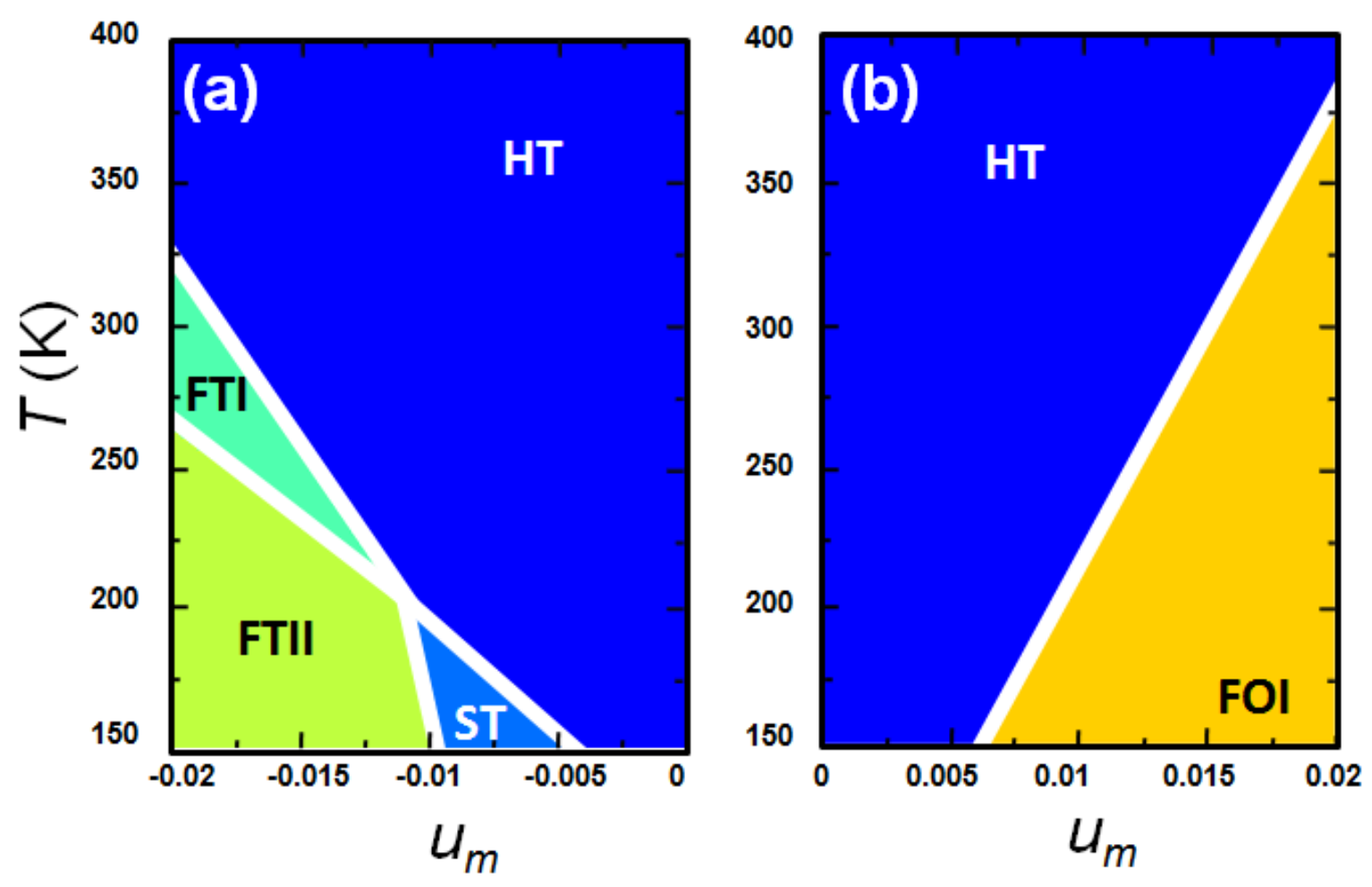

Figure 19. Misfit strain vs. temperature phase diagram of epitaxial monodomain (001) $\mathrm{SrTiO}_{3}$ films. The order parameters of the phases appearing in this map are: HT: $P_{1}=P_{2}=P_{3}=0, q_{1}=q_{2}=q_{3}=0$; ST: $P_{1}=P_{2}=P_{3}=0, q_{1}=q_{2}=0, q_{3} \neq 0$; FTI: $P_{1}=P_{2}=0, P_{3} \neq 0$, $q_{1}=q_{2}=q_{3}=0$; FTII: $P_{1}=P_{2}=0, P_{3} \neq 0, q_{1}=q_{2}=0, q_{3} \neq 0$; FOI: $\left|P_{1}\right|=\left|P_{2}\right| \neq 0, P_{3}=0, q_{1}=q_{2}=q_{3}=0$.

Figure 19 shows that, depending on $u_{m}$ and $T$, three ferroelectric phases (FTI, FTII, and FOI) can be stabilized by the lattice mismatch between the film and the substrate. For example, at $T=225 \mathrm{~K}$ and $0.0127<u_{m}<0.0106$, the HT phase is stable. The HT phase is a tetragonally distorted but non-polar variation of the parent cubic $(\mathrm{Pm} \overline{3} \mathrm{~m})$ phase. The tetragonality [i.e., $(c-a) / a$ where $c, a$ are the lattice parameters of the HT phase] is positive (negative) for $u_{m}<0\left(u_{m}>0\right)$ and is zero for $u_{m}=0$ for which $c=a=a_{0}$ where $a_{0}$ is the lattice parameter of unconstrained STO. For tensile misfit strains $u_{m}>0.0106$, the ferroelectric FOI phase (which has an equi-biaxial in-plane spontaneous polarization) stabilizes, while for compressive misfit strains $u_{m}<-0.0127$, the ferroelectric FTI phase is stabilized with an out- 
of-plane spontaneous polarization along the [001] direction. At this temperature, a transition to the FTII phase occurs for compressive misfit strains $u_{m}<-0.0144$. This transformation involves the rotation of the $\mathrm{TiO}_{6}$ octahedra characterized by the structural order parameter $q_{\mathrm{i}}$ and produces a change in the magnitude of polarization along the [001] direction. Hence, the magnitude of the polarization in any of the three ferroelectric phases depends on both $u_{m}$ and $T$.

The adiabatic temperature change $\Delta T$ for the ferroelectric phases can be explicitly calculated from Eq. (56). Here, the volumetric specific heat $C_{E}^{0}\left(T, E_{i}, u_{m}\right)$ was estimated by adding the computed zero-field values of the excess specific heat to the lattice contributions taken from experimental data [86].

A MIM construct having a (001) epitaxial STO film sandwiched between uniform metallic electrodes is considered first. For this configuration, $E_{i}=\left[0,0, E_{3}\right]$ and it is assumed that the bottom electrode is grown pseudomorphically onto the substrate so that both the sign and magnitude of $u_{m}$ are entirely controlled by the mismatch between the film and the substrate. As can be appreciated from Figure 19, compressive misfit strains favor the ferroelectric phases FTI and FTII while tensile misfit strains favor the ferroelectric phase FOI. Because both the HT and ST phases are non-polar, and because the component of polarization $P_{3}=0$ is parallel to the field direction $E_{3}$, the region of interest is restricted to compressive misfit strains $u_{m}<-0.01$. This is illustrated in Figure 20(a), which shows a twodimensional pseudo-color plot of the adiabatic temperature change $\Delta T$ as a function of $u_{m}$ and $T$ for a particular choice of bias field $E_{a}=50 \mathrm{kV} / \mathrm{cm}$ and field change $\Delta E=120 \mathrm{kV} / \mathrm{cm}$. 

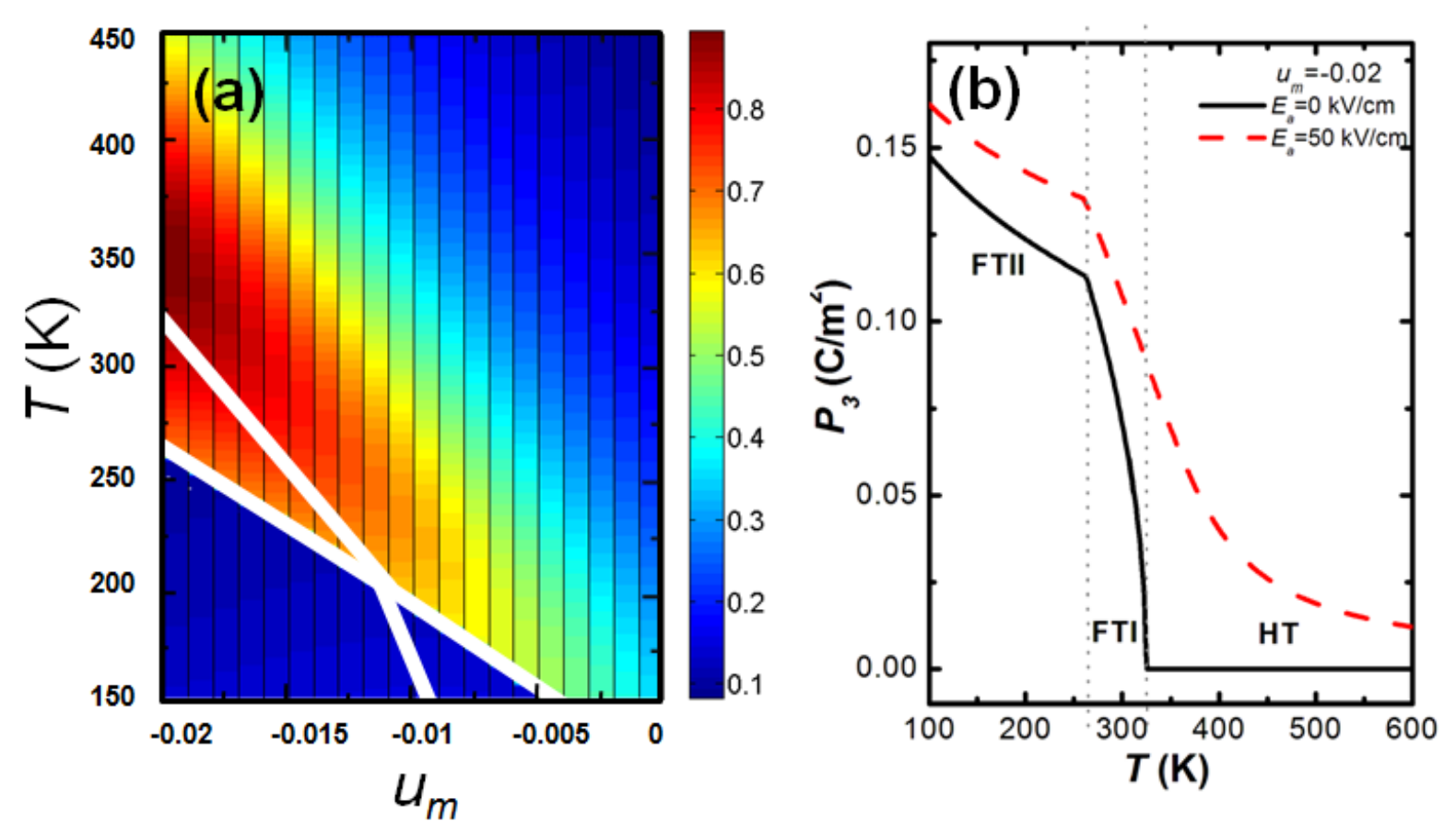

Figure 20. (a) The adiabatic temperature change $\Delta T$ of an epitaxial (001) $\mathrm{SrTiO}_{3}$ film in a MIM configuration as a function of $u_{m}$ and $T$ for $E_{a}=50 \mathrm{kV} / \mathrm{cm}$ and $\Delta E=120 \mathrm{kV} / \mathrm{cm}$; (b) the out-of plane polarization $P_{3}$ as a function of temperature at $u_{m}=\mathbf{- 0 . 0 2}$ for $E_{a}=0$ $\mathrm{kV} / \mathrm{cm}$ and $E_{a}=50 \mathrm{kV} / \mathrm{cm}$.

As expected for conventional ferroelectric materials such as $\mathrm{BaTiO}_{3}$ and $\mathrm{PbTiO}_{3}$ [21], the largest electrocaloric response occurs near the paraelectric to ferroelectric (HT-FTII) phase transition $(T=350 \mathrm{~K})$ where the $P_{3}(T)$ curve experiences an inflection point and the derivative $\partial P_{3} / \partial T$ passes through a steep minimum. However, at the comparatively low field level of $120 \mathrm{kV} / \mathrm{cm}$, the maximum adiabatic temperature change is modest, $\Delta T<1 \mathrm{~K}$. The reason for this can be understood from Figure 20(b) where it is seen that the polarization induced along [001] by a field $E_{3}=120 \mathrm{kV} / \mathrm{cm}$ is quite small. It is further apparent that the electrocaloric response is not significantly enhanced near the FTI-FTII phase boundary, because at this transition $P_{3}(T)$ shows only a small change in slope that is accompanied by comparatively small but discontinuous change in $\partial P_{3} / \partial T$. It is evident from Figure 20 that to 
obtain a larger $\Delta T$, the field strength and/or bias field must be increased. Figure 21 shows how $\Delta T$ varies as a function of field change $\Delta E$ at $\mathrm{RT}$. It is seen in this figure that for field changes $\Delta E=1200 \mathrm{kV} / \mathrm{cm}$, a large $\Delta T$ of $\sim 5 \mathrm{~K}$ can be achieved in STO films at RT. As a point of reference, at these field levels, the electrocaloric response for [001] STO in a MIM configuration is closely comparable to that observed in high-quality relaxor PMN-PT films [87] near the temperature of the Curie maximum ( 350 K). From this it can be concluded that, as shown for $\mathrm{BaTiO}_{3}[21]$, a relatively small bias field $(\sim 50 \mathrm{kV} / \mathrm{cm})$ is sufficient to destroy the discontinuity in polarization at the HT-FTII transition.

Finally, we show that by using an IDE configuration, it is possible to apply in-plane electric fields [88] and by so doing access the HT-FOI phase transformation that occurs under tensile misfit strains. Two IDE configurations are considered, one for which $E_{i}=$ $\left[E_{1}, 0,0\right]$ (or $\left[0, E_{2}, 0\right]$ ) oriented along [100] (or [010]) and a second for which $E_{i}=\left[E_{1} / \sqrt{2}, E_{1} /\right.$ $\sqrt{2}, 0]$ oriented along [110]. Pseudo-color plots of the adiabatic temperature change as functions of misfit strain and temperature are shown in Figure 22 and Figure 23 under the same conditions as the MIM configuration shown in Figure 20 (bias field $E_{a}=50 \mathrm{kV} / \mathrm{cm}$ and field change $\Delta E=120 \mathrm{kV} / \mathrm{cm})$. 


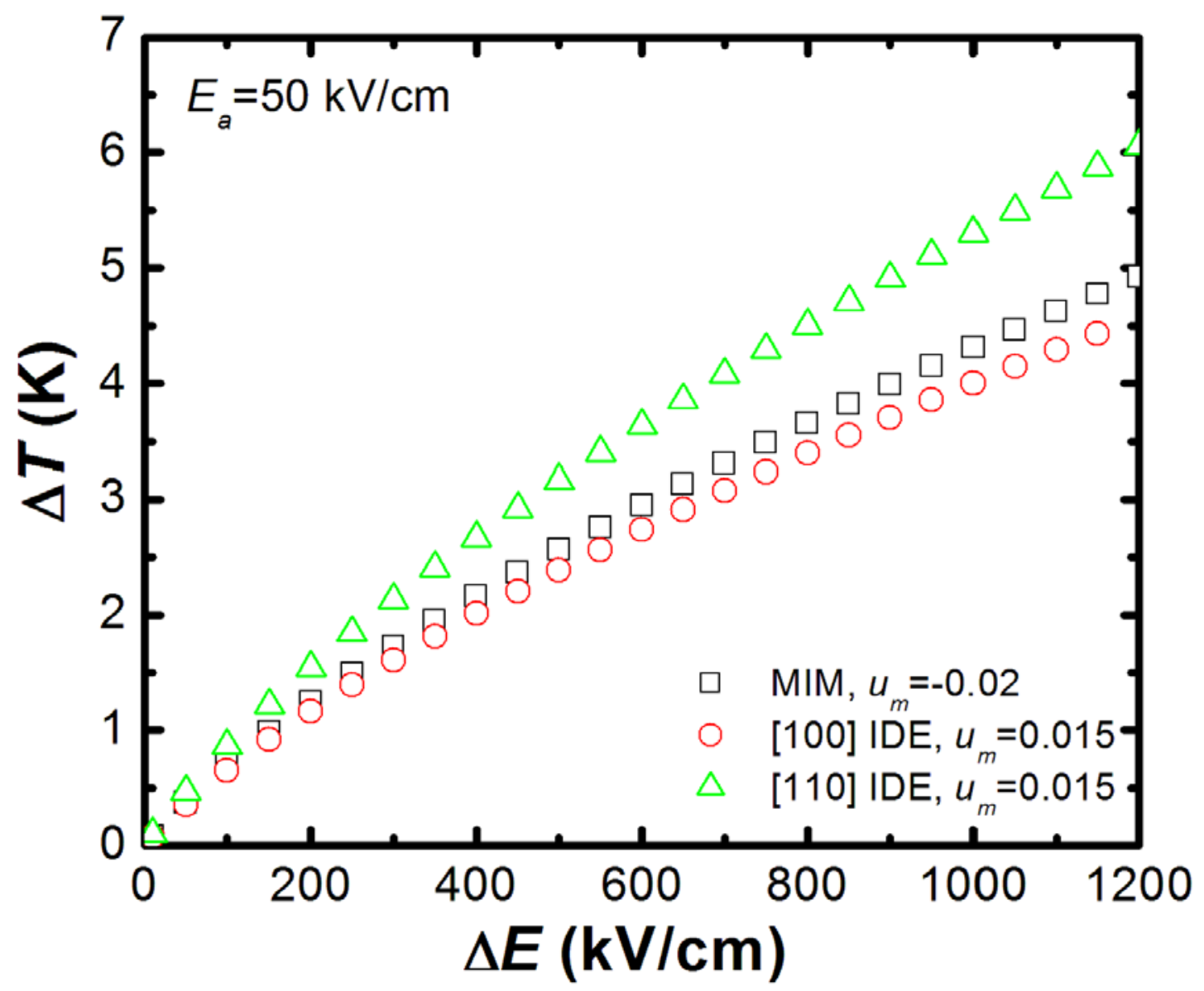

Figure 21. The room-temperature adiabatic temperature change $\Delta T$ of epitaxial (001) $\mathrm{SrTiO}_{3}$ films with MIM ( $u_{m}=-\mathbf{0 . 0 2 0}$, open squares) and [100]/[010] and [110] IDE configurations $\left(u_{m}=0.015\right.$, open circles and triangles, respectively) as a function of $\Delta E$ for $E_{a}=50 \mathrm{kV} / \mathrm{cm}$. 


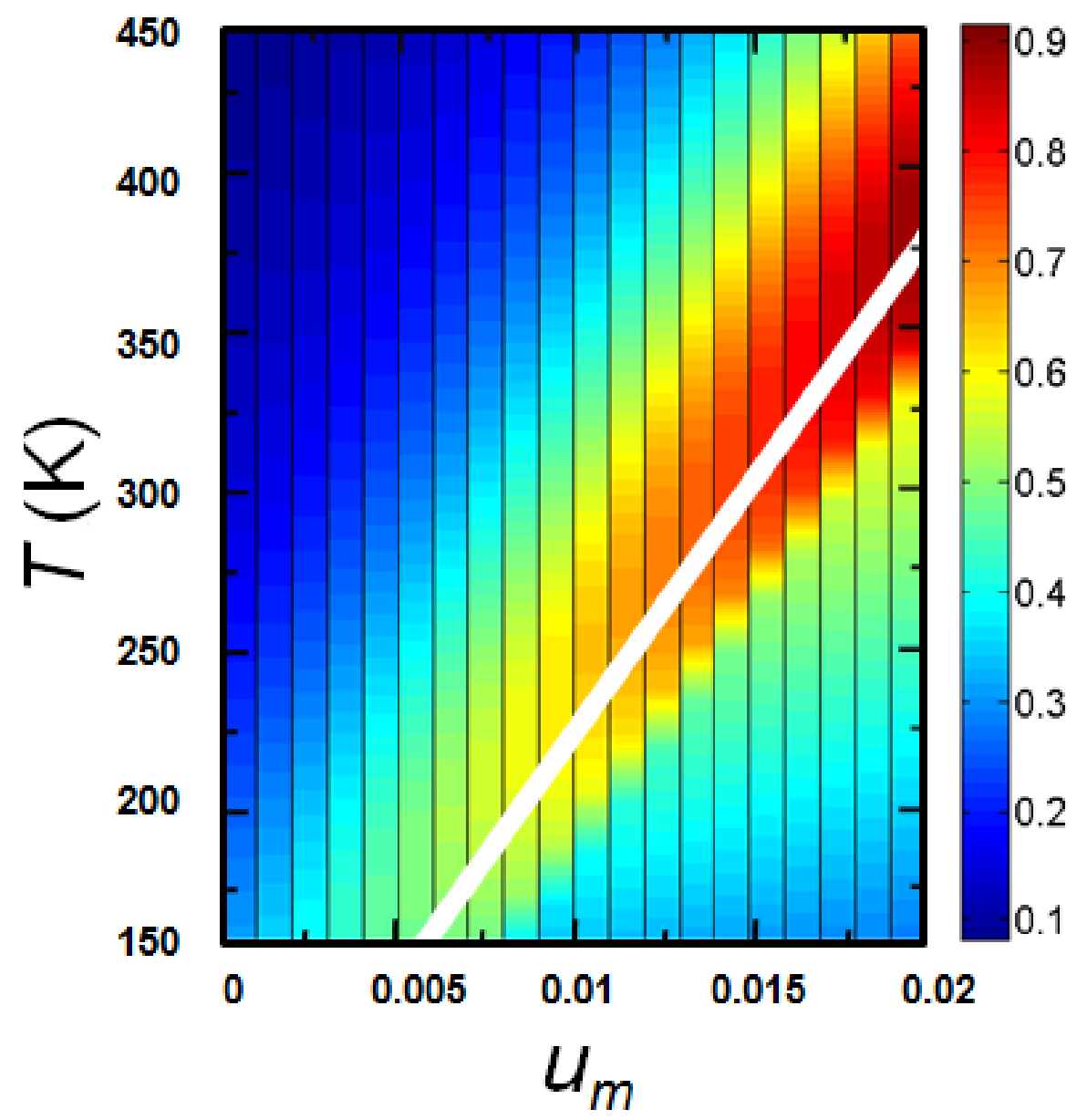

Figure 22. The adiabatic temperature change $\Delta T$ of an epitaxial (001) $\mathrm{SrTiO}_{3}$ film in a [100] IDE configuration as a function of $u_{m}$ and $T$ for $E_{a}=50 \mathrm{kV} / \mathrm{cm}$ and $\Delta E=120 \mathrm{kV} / \mathrm{cm}$.

As seen in Figure 22, the largest electrocaloric response $(\Delta T=0.9 \mathrm{~K})$ for the configuration with $E_{i}=\left[E_{1}, 0,0\right]$ occurs near the HT-FOI phase transformation, due to the strong coupling between the in-plane electrical field and the in-plane spontaneous polarization $P_{1}$ or $\left(P_{2}\right)$ of the FOI phase. For an IDE configuration with $E_{i}=\left[E_{1} / \sqrt{2}, E_{1} / \sqrt{2}\right.$ ,0], the largest ele response $(\Delta T=1.2 \mathrm{~K})$ is about $30 \%$ higher than for the configuration with $E_{i}=\left[E_{1}, 0,0\right]$. This can be explained by the fact that for the FOI phase with [100] or [010] 
IDEs, the electric field only induces polarization along one of the components $\left(P_{1}\right.$ or $\left.P_{2}\right)$, while for [110] IDEs, the applied field induces polarization in both components $P_{1}$ and $P_{2}$ with a magnitude of $|P|=\sqrt{P_{1}^{2}+P_{2}^{2}}$. Comparing the results presented in Figure 20, Figure 22, and Figure 23 it is seen that, under equivalent electrical boundary conditions, both MIM and IDE configurations have comparable electrocaloric responses ( $\Delta T \sim 1 \mathrm{~K})$ at $\mathrm{RT}$ if the misfit strain is adjusted such this temperature lies near either the HT-FTI or the HT-FOI phase transformation. The electrocaloric response as a function $\Delta E$ for the two IDE geometries is compared with that of the MIM configuration in Figure 21. As seen in the figure, all three configurations can produce a large $\Delta T(\sim 5 \mathrm{~K})$ at fields greater than 1000 $\mathrm{kV} / \mathrm{cm}$. As expected, the response is slightly larger for the [110] configuration compared with the [100] IDE or MIM. 


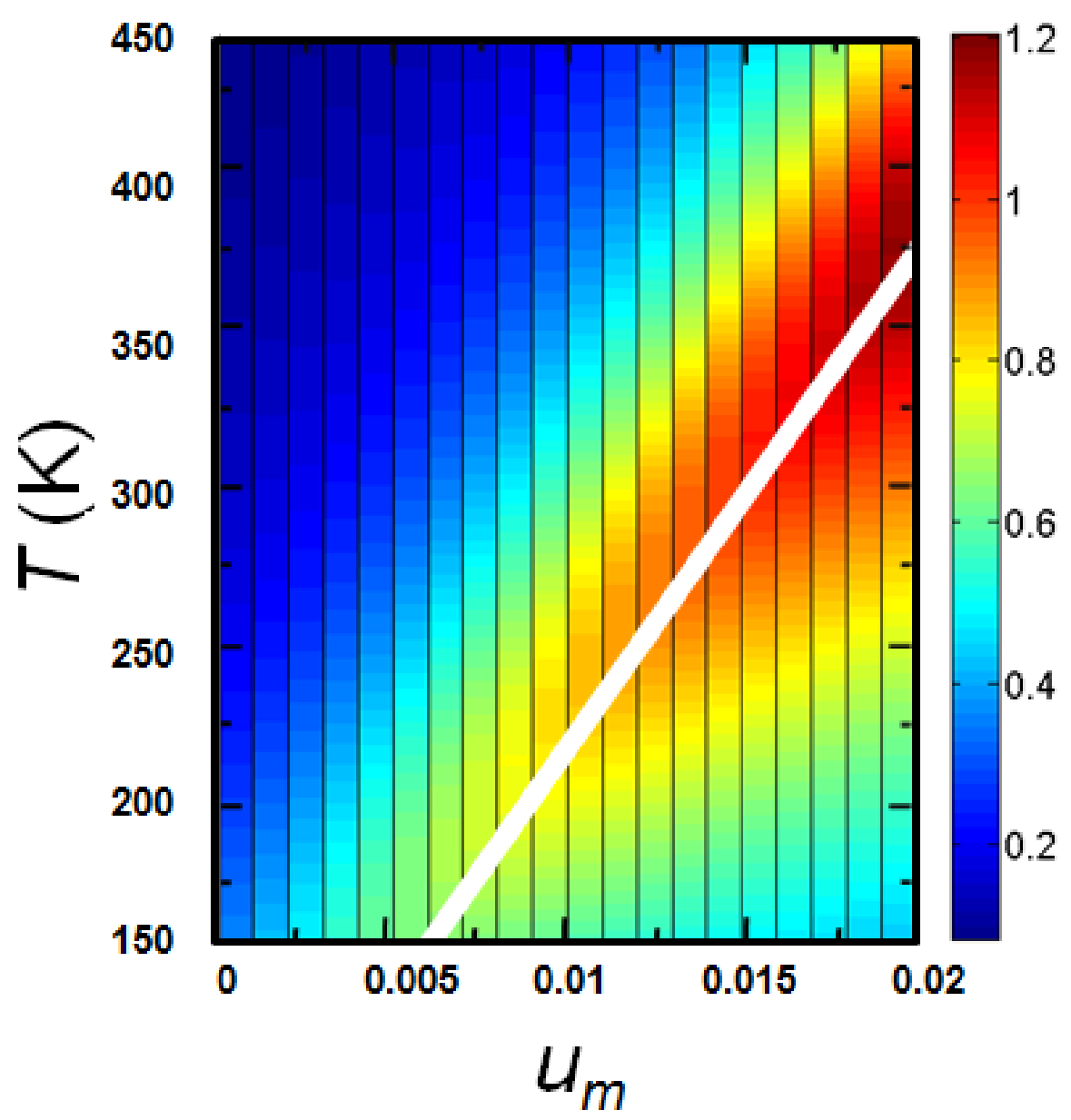

Figure 23. The adiabatic temperature change $\Delta T$ of an epitaxial (001) $\mathrm{SrTiO}_{3}$ film in a [110] IDE configuration as a function of $u_{m}$ and $T$ for $E_{a}=50 \mathrm{kV} / \mathrm{cm}$ and $\Delta E=120 \mathrm{kV} / \mathrm{cm}$. 


\section{CHAPTER 8}

\section{SUMMARY AND CONCULSIONS}

From the analysis presented above, several conclusions can be drawn that are relevant in investigations of the electrocaloric properties of ferroelectric materials.

\subsection{Influence of Composition and Phase Transition Order}

For ferroelectrics such as BTO and PTO displaying weak first-order transitions close to second-order, the use of a bias field is most likely essential in obtaining reproducible results under experimental conditions. For a given set of mechanical boundary constraints, the magnitude of this bias field would be comparable to that at the electrical critical point. The application of a bias field of greater magnitude reduces the sensitivity of the electrocaloric effect to temperature, but of course, also reduces the magnitude of the effect itself. Materials with stronger first-order phase transitions will naturally require a higher bias field. Under conditions of perfect mechanical clamping, the weak first-order phase change as displayed by BTO and PTO will become of second-order, and in this instance the electrical critical point is coincident with the Curie temperature. As a consequence, a lower bias field will be required to produce a comparable set of measurement conditions. Alternatively, in solid solution ferroelectric systems, both the Curie temperature and the first-order character of the ferroelectric phase transition also change continuously with composition. The cooperative effect on the phase transition of mechanical boundary constraints, electric drive conditions and composition, is an important consideration in interpreting experimental observations of electrothermal phenomena in ferroelectric thin film materials selected to have Curie points located in a desired temperature range. 


\subsection{Influence of Thermal Stresses}

We used a non-linear thermodynamic model to investigate the role of thermal stresses on the RT pyroelectric properties of (001)-textured polycrystalline BST films with four different compositions (BST 60/40, 70/30, 80/20 and 90/10) on three IC-friendly substrates: Si, (0001) sapphire, and ( $\overline{1} \overline{1} 20)$ sapphire. The main findings of this study are summarized below:

- $\quad$ Due to the CTE difference between the BST film compositions and the substrate materials analyzed in this study, the in-plane tensile thermal strain in BST increases with increasing deposition/processing temperature $T_{G}$. This leads to a depression of the paraelectric-ferroelectric phase transformation. As such, relatively lower deposition/processing temperature would result in better pyroelectric response.

- $\quad$ For BST 60/40 and 70/30 on all the substrates, the film is in the PE state for $T_{G}=25$ $800^{\circ} \mathrm{C}$ and the pyroelectric response is due to interplay of the electric field and thermal strain dependence of the dielectric constant and the $T_{G}$-dependence of the thermal strains. While the RT pyroelectric response of these films is expected to be relatively low $\left(0.02-0.05 \mu \mathrm{C} / \mathrm{cm}^{2} \mathrm{~K}\right.$ at $\sim 200 \mathrm{kV} / \mathrm{cm}$ ), at high applied fields the pyroelectric response does not display a significant dependence on the temperature at which the material was treated.

- $\quad$ BST 80/20 and BST 90/10 films may be in the ferroelectric state at RT depending on $T_{G}$. If $T_{G}$ can be adjusted such that it coincides with the FE-PE transition temperature, a relatively high bulk-like pyroelectric response $\left(0.5-1.0 \mu \mathrm{C} / \mathrm{cm}^{2} \mathrm{~K}\right)$ can be realized. Depending on the BST composition and the substrate material analyzed in this study, $T_{G}$ varies between $95^{\circ} \mathrm{C}$ (BST $80 / 20$ on $\mathrm{Si}$ ) and $294^{\circ} \mathrm{C}$ (BST 90/10 on $a$-sapphire). However, the pyroelectric 
response decreases sharply above and below these "critical” $T_{G}$ which requires a precise temperature control during processing.

Furthermore, we have shown that the thermal stresses that develop during processing can have a significant influence on the electrocaloric properties of thin film ferroelectric materials. The main effect of the thermal stresses is to shift the zero-field Curie temperature. If the stresses are tensile, the temperature at which the adiabatic temperature change is maximized shifts to lower temperature. For a particular choice of the ferroelectric material, the type of substrate, substrate orientation, and growth/annealing temperature can be adjusted to attain an adiabatic temperature change comparable to that of the intrinsic bulk response at temperatures far from the zero-field $T_{C}$ of the stress-free bulk material.

\subsection{Incipient Ferroelectric Materials}

We have computed the electrocaloric response of STO films as a function of the misfit strain, temperature, applied electric field strength, and electrode configuration. It was shown that for STO films on compressive substrates the electrocaloric response can be enhanced in a MIM configuration with uniform electrodes by exploiting the HT-FTI transition. At fields of $\sim 1000 \mathrm{kV} / \mathrm{cm}$ the computed temperature change $\Delta T=5 \mathrm{~K}$ is comparable to ferroelectric films near the Curie point. Alternatively, for STO films on tensile substrates the electrocaloric response can be enhanced by using an IDE configuration that exploits the HT-FOI transition, with the maximum response occurring for a [110] IDE orientation. Compared with MIM configurations, STO films utilizing an IDE configuration may offer possibilities to increase the electrocaloric response while minimizing the dead volume of electrodes. These results show that the strain-induced electrocaloric properties of 
incipient ferroelectrics are closely comparable to the measured electrocaloric response of conventional or relaxor ferroelectrics. 


\section{REFERENCES}

[1] R. Watton, Ferroelectrics 91, 87 (1989).

[2] R. W. Whatmore, P. C. Osbond, and N. M. Shorrocks, Ferroelectrics 76, 351 (1987).

[3] V. K. Pecharsky and K. A. Gschneidner Jr., J. Magn. Magn. Mater. 200, 44 (1999).

[4] F. Jona and G. Shirane, Ferroelectric Crystals. (Dover, New York, 1962).

[5] R. W. Whatmore, Q. Zhang, C. P. Shaw, R. A. Dorey, and J. R. Alcock, Phys. Scr. T129, 6 (2007).

[6] R. W. Whatmore, Rep. Prog. Phys. 49, 1335 (1986).

[7] P. Muralt, Rep. Prog. Phys. 64, 1339 (2001).

[8] A. Rogalski, Infrared Phys. Technol. 43, 187 (2002).

[9] M. H. Lee, R. Guo, and A. S. Bhalla, J. Electroceram. 2, 229 (1998).

[10] B. Neese, B. Chu, S. -G. Lu, Y. Wang, E. Furman, and Q. M. Zhang, Science 321, 821 (2008).

[11] P. Bao, T. J. Jackson, X. Wang, and M. J. Lancaster, J. Phys. D: Appl. Phys. 41, 063001 (2008).

[12] M. W. Cole, C. Hubbard, E. Ngo, M. Ervin, M. Wood, and R. G. Geyer, J. Appl. Phys. 92, 475 (2002).

[13] N. K. Pervez, P. J. Hansen, and R. A. York, Appl. Phys. Lett. 85, 4451 (2004).

[14] E. A. Fardin, A. S. Holland, K. Ghorbani, E. K. Akdogan, W. K. Simon, A. Safari, and J. Y. Wang, Appl. Phys. Lett. 89, 182907 (2006).

[15] D. M. Potrepka, S. Hirsch, M. W. Cole, W. D. Nothwang, S. Zhong, and S. P. Alpay, J. Appl. Phys. 99, 014108 (2006).

[16] S. L. Delprat, C. Durand, J. Oh, M. Chaker, and K. Wu, Appl. Phys. Lett. 91, 063513 (2007).

[17] B. Xiao, H. Liu, V. Avrutin, J. H. Leach, E. Rowe, H. Liu, U. Ozgur, H. Morkoc, W. Chang, L. M. B. Alldredge, S. W. Kirchoefer, and J. M. Pond, Appl. Phys. Lett. 95, 212901 (2009).

[18] W. Hu, C. Yang, X. Liu, W. He, and X. Tang, J. Mater. Sci. - Mater. Electron. 19, 61 (2008).

[19] S. N. Song, J. W. Zhai, and X. Yao, J. Cryst. Growth 308, 223 (2007). 
[20] N. Xiong, S. Jiang, Y. Li, L. Tan, and R. Li, Appl. Phys. Lett. 93, 232905 (2008).

[21] Y. Zhang, G. Wang, Y. Chen, L. Yang, F. Cao, and X. Dong, J. Am. Ceram. Soc. 92, 2795 (2009).

[22] P. A. Fleury, J. F. Scott, and J. M. Worlock, Phys. Rev. Lett. 21, 16 (1968).

[23] G. Shirane and Y. Yamada, Phys. Rev. 177, 858 (1969).

[24] J. C. Slonczewski and H. Thomas, Phys. Rev. B 1, 3599 (1970).

[25] H. Uwe and T. Sakudo, Phys. Rev. B 13, 271 (1976).

[26] J. Hemberger, M. Nicklas, R. Viana, P. Lunkenheimer, A. Loidl, and R. Bohmer, J. Phys.: Condens. Matter 8, 4673 (1996).

[27] J. G. Bednorz and K. A. Muller, Phys. Rev. Lett. 52, 2289 (1984).

[28] J. H. Haeni, P. Irvin, W. Chang, R. Uecker, P. Reiche, Y. L. Li, S. Choudhury, W. Tian, M. E. Hawley, B. Craigo, A. K. Tagantsev, X. Q. Pan, S. K. Streiffer, L. Q. Chen, S. W. Kirchoefer, J. Levy, and D. G. Schlom, Nature 430, 758 (2004).

[29] N. A. Pertsev, A. K. Tagantsev, and N. Setter, Phys. Rev. B 61, R825 (2000).

[30] N. A. Pertsev, A. K. Tagantsev, and N. Setter, Phys. Rev. B 65, 219901 (2002).

[31] A. I. Kingon, J.-P. Maria, and S. K. Streiffer, Nature 406, 1032 (2000).

[32] P. C. Joshi and S. B. Krupanidhi, J. Appl. Phys. 73, 7627 (1993).

[33] F. M. Pontes, E. J. H. Lee, E. R. Leite, and E. Longo, J. Mater. Sci. 35, 4783 (2000).

[34] M. A. Saifi and L. E. Cross, Phys. Rev. B 2, 677 (1970).

[35] A. Sharma, Z. -G. Ban, S. P. Alpay, and J. V. Mantese, Appl. Phys. Lett. 84, 4959 (2004).

[36] A. Sharma, Z. -G. Ban, S. P. Alpay, and J. V. Mantese, J. Appl. Phys. 95, 3618 (2004).

[37] Hai-Xia Cao, Veng Cheong Lo, and Zhen-Ya Li, J. Appl. Phys. 101, 014113 (2007).

[38] G. Akcay, S. P. Alpay, G. A. Rossetti Jr., and J. F. Scott, J. Appl. Phys. 103, 024104 (2008).

[39] A. S. Mischenko, Q. Zhang, J. F. Scott, R. W. Whatmore, and N. D. Mathur, Science 311, 1270 (2006).

[40] A. Sharma, Z. -G. Ban, S. P. Alpay, and J. V. Mantese, J. Appl. Phys. 95, 3618 (2004).

[41] Z. -G. Ban and S. P. Alpay, Appl. Phys. Lett. 82, 3499 (2003). 
[42] M. W. Cole, P. C. Joshi, C. W. Hubbard, and E. Ngo, Integr. Ferroelectr. 83, 99 (2006).

[43] E. Fatuzzo, H. Kiess, and R. Nitsche, J. Appl. Phys. 37, 510 (1966).

[44] J. D. Childress, J. Appl. Phys. 33, 1793 (1962).

[45] P. D. Thacher, J. Appl. Phys. 39, 1996 (1968).

[46] G. Akcay, S. P. Alpay, J. V. Mantese, and G. A. Rossetti, Jr., Appl. Phys. Lett. 90, 252909 (2007).

[47] L. J. Dunne, M. Valant, G. Manos, A. -K. Axelsson, and N. Alford, Appl. Phys. Lett. 93, 122906 (2008).

[48] S. Prosandeev, I. Ponomareva, and L. Bellaiche, Phys. Rev. B 78, 052103 (2008).

[49] J. H. Qiu and Q. Jiang, J. Appl. Phys. 103, 034119 (2008).

[50] G. Akcay, S. P. Alpay, G. A. Rossetti, Jr., and J. F. Scott, J. Appl. Phys. 103, 024104 (2008).

[51] J. Karthik and L. W. Martin, Appl. Phys. Lett. 99, 032904 (2011).

[52] Q. Peng and R. E. Cohen, Phys. Rev. B 83, 220103 (2011).

[53] B. Li, J. B. Wang, X. L. Zhong, F. Wang, and Y. C. Zhou, J. Appl. Phys. 107, 014109 (2010).

[54] S. Lisenkov and I. Ponomareva, Phys. Rev. B 80, 140102 (2009).

[55] S. Prosandeev, I. Ponomareva, and L. Bellaiche, Phys. Rev. B 78, 052103 (2008).

[56] J. Zhang, A. A. Heitmann, S. P. Alpay, and G. A. Rossetti, Jr., J. Mater. Sci. 44, 5263 (2009).

[57] S. Lisenkov and I . Ponomareva, Phys. Rev. B 80, 140102 (2009).

[58] T. M. Correia, J. S. Young, R. W. Whatmore, J. F. Scott, N. D. Mathur, and Q. Zhang, Appl. Phys. Lett. 95, 182904 (2009).

[59] H. Chen, T. -L. Ren, X. -M. Wu, Y. Yang, and L. -T. Liu, Appl. Phys. Lett. 94, 182902 (2009).

[60] S. Kar-Narayan and N. D. Mathur, J. Phys. D: Appl. Phys. 43, 032002 (2010).

[61] G. Sebald, S. Pruvost, L. Seveyrat, L. Lebrun, D. Guyomar, and B. Guiffard, J. Eur. Ceram. Soc. 27, 4021 (2007).

[62] D. G. Rabus, Z. X. Bian, and A. Shakouri, Ieee Photonic Tech L 17 (9), 1770 (2005). 
[63] D. G. Schlom, L.-Q. Chen, C.-B. Eom, K. M. Rabe, S. K. Streiffer, and J.-M. Triscone, Annu. Rev. Mater. Res. 37, 589 (2007).

[64] Z.-G. Ban and S. P. Alpay, J. Appl. Phys. 91, 9288 (2002).

[65] S. P. Alpay, I. B. Misirlioglu, A. Sharma, and Z.-G. Ban, J. Appl. Phys. 95, 8118 (2004).

[66] S. Zhong, S. P. Alpay, Z.-G. Ban, and J. V. Mantese, Appl. Phys. Lett. 86, 092903 (2005).

[67] Boris A. Strukov and A. P. Levanyuk, Ferroelectric phenomena in crystals : physical foundations. (Springer, Berlin ; New York, 1998), pp.x.

[68] Neil Mathur and Alexandr Mischenko, U.S. Patent No. WO 2006/056809 A1 (Jun. 1 2006).

[69] S. F. Karmanenko, O. V. Pakhomov, A. M. Prudan, A. S. Starkov, and A. Eskov, J. Eur. Ceram. Soc. 27, 3109 (2007).

[70] J. He, J. Chen, J. T. Wang, and B. Hua, Int. J. Therm. Sci. 42, 169 (2003).

[71] L. Feigl, S. J. Zheng, B. I. Birajdar, B. J. Rodriguez, Y. L. Zhu, M. Alexe, and D. Hesse, J. Phys. D: Appl. Phys. 42, 085305 (2009).

[72] Robert E. Newnham, Properties of materials : anisotropy, symmetry, structure. (Oxford University Press, Oxford ; New York, 2005), pp.xii.

[73] A. F. Devonshire, Philos. Mag. 40, 1040 (1949).

[74] A. F. Devonshire, Philos. Mag. 42, 1065 (1951).

[75] N. A. Pertsev, A. G. Zembilgotov, and A. K. Tagantsev, Phys. Rev. Lett. 80, 1988 (1998).

[76] Q. Y. Qiu, V. Nagarajan, and S. P. Alpay, Phys. Rev. B 78, 064117 (2008).

[77] Y. Okada and Y. Tokumaru, J. Appl. Phys. 56, 314 (1984).

[78] Ronald G. Munro, J. Am. Ceram. Soc. 80, 1919 (1997).

[79] A. Sharma, Z. -G. Ban, S. P. Alpay, and J. V. Mantese, Appl. Phys. Lett. 85, 985 (2004).

[80] J. V. Mantese, A. L. Micheli, N. W. Schubring, M. W. Putty, M. P. Thompson, S. Chang, J. R. Troxell, and L. Oberdier, Appl. Phys. Lett. 90, 113503 (2007).

[81] A. G. Zembilgotov, N. A. Pertsev, U. Bottger, and R. Waser, Appl. Phys. Lett. 86, 052903 (2005). 
[82] J. Zhang, I. B. Misirlioglu, S. P. Alpay, and Jr. G. A. Rossetti, Appl. Phys. Lett. 100, 222909 (2012).

[83] B. Li, W. J. Ren, X. W. Wang, H. Meng, X. G. Liu, Z. J. Wang, and Z. D. Zhang, Appl. Phys. Lett. 96, 102903 (2010).

[84] I. B. Misirlioglu, S. P. Alpay, M. Aindow, and V. Nagarajan, Appl. Phys. Lett. 88, 102906 (2006).

[85] J. S. Speck, A. Seifert, W. Pompe, and R. Ramesh, J. Appl. Phys. 76, 466 (1994).

[86] D. Ligny and P. Richet, Phys. Rev. B 53, 3013 (1996).

[87] A. S. Mischenko, Q. Zhang, R. W. Whatmore, J. F. Scott, and N. D. Mathur, Appl. Phys. Lett. 89, 242912 (2006).

[88] W. K. Simon, E. K. Akdogan, A. Safari, and J. A. Bellotti, Appl. Phys. Lett. 87, 082906 (2005). 